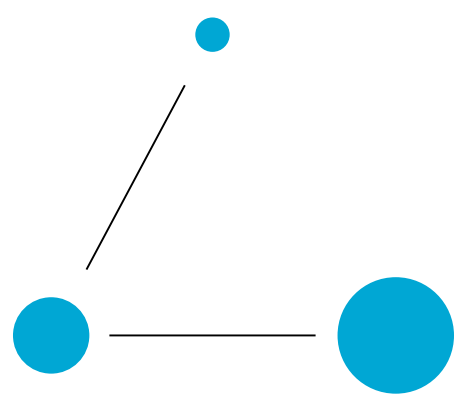

A N N A L E S

HENRI LEBESGUE

\title{
ÉLISE JANVRESSE
}

EMMANUEL ROY

\section{THIERRY DE LA RUE}

\section{NEARLY FINITE CHACON \\ TRANSFORMATION}

\section{TRANSFORMATION DE CHACON PRESQUE F I N I E}

Abstract. - We construct an infinite measure preserving version of Chacon transformation, and prove that it has a property similar to Minimal Self-Joinings in finite measure: its Cartesian powers have as few invariant Radon measures as possible.

RÉsumÉ. - Nous construisons une version de la transformation de Chacon en mesure infinie, et prouvons qu'elle satisfait une propriété similaire aux autocouplages minimaux en mesure finie : ses puissances cartésiennes ont aussi peu de mesures de Radon invariantes que possible.

\section{Introduction}

\subsection{Motivations}

The purpose of this work is to continue the study, started in [JRdlR18] and [Dan18], of what the Minimal Self-Joinings (MSJ) property could be in the setting of infinitemeasure preserving transformations. We want here to construct an infinite measure

Keywords: Chacon infinite measure preserving transformation, rank-one transformation, joinings. 2010 Mathematics Subject Classification: 37A40, 37A05.

DOI: https://doi.org/10.5802/ahl.21

(*) Research partially supported by French research group GeoSto (CNRS-GDR3477). 
preserving transformation whose Cartesian powers have as few invariant measures as possible. As in the aforementioned papers, we restrict ourselves to Radon measures (giving finite mass to compact sets), since in general there are excessively many infinite invariant measures for a given transformation (think of the sum of Dirac masses along an orbit).

A first attempt in this direction was to consider the so-called infinite Chacon transformation introduced in [AFS97]. Indeed, the construction of this infinite measure preserving rank-one transformation is strongly inspired by the classical finite measure preserving Chacon transformation, which enjoys the MSJ property [dJRS80]. The identification of invariant measures for Cartesian powers of the infinite Chacon transformation was the object of our previous work [JRdlR18]. In addition to the products of graph measures arising from powers of the transformation (see the beginning of Section 3.3 for details), we found in the case of infinite Chacon some kind of unexpected invariant measures, the so-called weird measures. These weird measures have marginals which are singular with respect to the original invariant measure, but it is shown in [Dan18, Example 5.4] that an appropriate convex combination of weird measures can have absolutely continuous marginals.

We propose here another rank-one transformation, which we call the nearly finite Chacon transformation, hereafter denoted by $T$. Although it preserves an infinite measure $\mu$, its construction is designed to mimic as much as possible the behaviour of the classical Chacon transformation, so that the phenomenon of weird measures disappears. Our main result, Theorem 3.10, is the following: there exists a $\mu$-conull set $X_{\infty}$ such that, for each $d \geqslant 1$, the ergodic $T^{\times d}$-invariant Radon measures on $X_{\infty}^{d}$ are the product measure $\mu^{\otimes d}$ and products of graph measures arising from

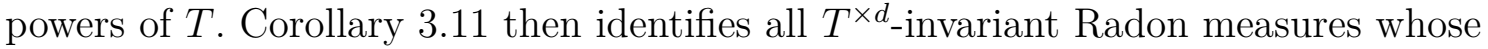
marginals are absolutely continuous with respect to $\mu$ as sums of countably many ergodic components which are of the form given in the theorem.

Beyond the question of the MSJ property in the infinite measure world, the example presented in this paper is also of crucial importance in the study of Poisson suspensions. A Poisson suspension is a finite measure preserving dynamical system constructed from an infinite measure preserving system: a state of the space is a realization of a Poisson point process whose intensity is the infinite invariant measure, and each random point evolves according to the dynamics of the infinite measure preserving transformation (we refer to [Roy07] for a complete presentation of Poisson suspensions). Although of different nature, Poisson suspensions share surprising properties with another category of finite measure preserving dynamical systems of probabilistic origin: Gaussian dynamical systems, which are constructed from finite measures on the circle. A beautiful theory has been developed in [LPT00], concerning a special class of Gaussian systems called GAGs (a French acronym for Gaussian systems with Gaussian self-joinings). The keystone for the construction of a GAG system is a striking theorem due to Foiaş and Strătilă [FS67]: if a measure supported on a Kronecker subset of the circle appears as the spectral measure of some ergodic stationary process, then this process is Gaussian. The Poisson counterpart of GAG, called $\mathcal{P} a \mathcal{P}$ (Poisson suspension with Poisson self-joinings) is presented in [JRdlR17], where the construction of a $\mathcal{P} a \mathcal{P}$ example relies on a theorem $\grave{a}$ la 
Foiaş-Strătilă (see [JRdlR17, Theorem 3.4]). Roughly speaking, according to this theorem, if some ergodic point process evolves under a dynamics directed by an infinite measure preserving transformation with special properties, then this point process is Poissonian. The special properties needed here are precisely those given by Corollary 3.11. Therefore, systems enjoying those properties play in the theory of Poisson suspensions the same role as measures supported on Kronecker subset in the setting of Gaussian systems.

For some applications in the study of Poisson suspensions developed in [JRdlR17], we also need an additional property which is the existence of a measurable law of large numbers. Proposition 8.4 shows that the nearly finite Chacon transformation satisfies a stronger property called rational ergodicity.

\subsection{Roadmap of the paper}

Section 2 is devoted to the construction of the nearly finite Chacon transformation, and to first elementary results. For pedagogical reasons, we start in Section 2.1 by defining the nearly finite Chacon transformation with the cutting-and-stacking method on $\mathbb{R}_{+}$equipped with the Lebesgue measure, as it is easier to visualize the structure of the Rokhlin towers in this setting. Most steps of the construction are identical to construction of the classical Chacon transformation. There is just a fast increasing sequence $\left(n_{\ell}\right)$ of integers such that each $n_{\ell}$-th step of the construction differs from classical Chacon, which ensures that the invariant measure has infinite mass. Then we turn in Section 2.2 to a more convenient (but isomorphic) model for our purposes, which is a transformation $T$ on a set $X$ of sequences on a countable alphabet. In Section 2.3, we describe basic properties of a typical point with respect to the invariant measure $\mu$, and define the conull set $X_{\infty}$ referred to in Theorem 3.10.

Section 3 contains the main results concerning Radon measures on $X^{d}$ which are $T^{\times d}$-invariant. Section 3.1 first states some basic facts about Radon measures on $X^{d}$. We give a criterion for such a measure to be $T^{\times d}$-invariant (Lemma 3.2). We also define a notion of convergence of Radon measures (Definition 3.3), which is specially adapted to the formulation of Hopf's ratio ergodic theorem, and give useful lemmas concerning this convergence. In Section 3.2, we treat the easy case of totally dissipative measures: Proposition 3.9 eliminates the possibility of a totally dissipative $T^{\times d}$-invariant Radon measure supported on $X_{\infty}^{d}$. In Section 3.3, we state our main result (Theorem 3.10) and establish the bases of a proof by induction on $d$. At the end of Section 3.3, we fix once and for all a $T^{\times d}$-invariant Radon measure $\sigma$ supported on $X_{\infty}^{d}$, for some $d \geqslant 2$. The remainder of the paper is completely devoted to proving that either $\sigma$ is a graph measure arising from powers of $T$, or it can be decomposed as a product of two measures on which we can apply the induction hypothesis. In Section 3.4, we choose once and for all a $\sigma$-typical point $x \in X_{\infty}^{d}$, on the orbit of which we estimate the properties of $\sigma$. We also introduce in Definition 3.12 the central notion of $n$-crossings, which are finite subintervals of $\mathbb{Z}$ depending on the position of the orbit of the typical point $x$ with respect to the $n$-th Rokhlin tower of the rank-one construction. The analysis of the structure of those $n$-crossings constitutes the core of our proof. In Section 3.5, we provide a criterion 
for $\sigma$ to be a graph measure arising from powers of $T$, stated in terms of $n$-crossings (Proposition 3.17).

Section 4 is devoted to the proof of Proposition 4.1, which is a central result in the analysis of the structure of $n$-crossings. Section 4.1 describes a hierarchy of abstract subsets of $\mathbb{Z}$ and provides a lemma on the combinatorics of subsets in this hierarchy (Lemma 4.2). Then Section 4.2 explains how to apply this lemma to the structure of $n$-crossings. Section 4.3 provides a useful corollary of Proposition 4.1 in terms of the measure $\sigma$.

Section 5 is devoted to the study of the convergence of empirical measures, which are finite sums of Dirac masses on points on the orbit of $x$, corresponding to finite subsets of $\mathbb{Z}$. We provide two criteria, Proposition 5.7 and Proposition 5.9, for a sequence of such empirical measures to converge to $\sigma$.

In Section 6 we present the main tool used to decompose $\sigma$ as a product measure. We introduce the notion of twisting transformation (Definition 6.1), which is simply a transformation of $X^{d}$ acting as $T$ on some coordinates, and as Id on others. Based on a theorem from [JRdlR18], Proposition 6.2 shows that, if $\sigma$ is invariant by such a twisting transformation, then $\sigma$ decomposes as a product measure to which we can apply the induction hypothesis. Then Proposition 6.3 provides a criterion for $\sigma$ to be invariant by some twisting transformation.

All the preceding tools are used in Section 7, where the proof of Theorem 3.10 is completed. If the criterion given by Proposition 3.17 for $\sigma$ to be a graph measure fails, then for infinitely many integers $n$ there exists some $n$-crossing, not too far from 0 , with some special property. We treat several cases according to the positions of these integers with respect to the sequence $\left(n_{\ell}\right)$. With the help of Propositions 6.3 and 6.2 , we show that in all cases $\sigma$ decomposes as a product of two measures to which the induction hypothesis applies.

The last (short) section is devoted to some additional properties of the nearly finite Chacon transformation. We give some corollaries of the main result concerning the commutant and the factors of the transformation, and we also deal with rational ergodicity and the existence of a measurable law of large numbers.

\section{Construction of the nearly finite Chacon transformation}

\subsection{Cutting-and-stacking construction on $\mathbb{R}_{+}$}

As previously explained, this transformation is designed to mimic the classical finite measure preserving Chacon transformation as much as possible, yet it must preserve an infinite measure. The construction will make use of two predefined increasing sequences of integers: $1 \ll n_{1} \ll n_{2} \ll \cdots \ll n_{\ell} \ll \cdots$ and $\ell_{0}:=1 \ll \ell_{1} \ll \ell_{2} \ll$ $\cdots \ll \ell_{k} \ll \cdots$, satisfying some growth conditions to be specified later (see below conditions (2.1) and (2.2)). For each $\ell \geqslant 1$, there exists a unique integer $k \geqslant 0$ such that $\ell_{k} \leqslant \ell<\ell_{k+1}$, and we denote this integer by $k(\ell)$.

In the first step we consider the interval $[0,1)$, which is cut into three subintervals of equal length. We take the extra interval $[1,4 / 3)$ and stack it above the middle piece. Then we stack all these intervals left under right, getting a tower of height 
$h_{1}:=4$. The transformation $T$ maps each point to the point exactly above it in the tower. At this step $T$ is yet undefined on the top of the tower.

After step $n$ we have a tower of height $h_{n}$, called tower $n$, the levels of which are intervals of length $1 / 3^{n}$. These intervals are closed to the left and open to the right. At step $(n+1)$, tower $n$ is cut into three subcolumns of equal width. If $n \notin\left\{n_{\ell}: \ell \geqslant 1\right\}$, we do as in the standard finite measure preserving Chacon transformation: we add an extra interval of length $1 / 3^{n+1}$ above the middle subcolumn, and we stack the three subcolumns left under right to get tower $n+1$ of height $h_{n+1}=3 h_{n}+1$. If $n=n_{\ell}$ for some $\ell$, we add $h_{n-k(\ell)}$ extra intervals above each of the three subcolumns, and a further extra interval above the second subcolumn. Then we stack the three subcolumns left under right and get tower $n+1$ of height $h_{n+1}=3 h_{n}+3 h_{n-k(\ell)}+1$. (See Figure 2.1.)

At each step, we pick the extra intervals successively by taking the leftmost interval of desired length in the unused part of $\mathbb{R}_{+}$. Extra intervals used at step $n+1$ are called $(n+1)$-spacers.

We want the Lebesgue measure of tower $n$ to increase to infinity, which is easily satisfied provided the sequence $\ell_{k}$ grows sufficiently fast. Indeed, for each $n \geqslant 1$ we have $h_{n+1} \leqslant 6 h_{n}+1 \leqslant 7 h_{n}$, whence $h_{n} / h_{n+1} \geqslant 1 / 7$. It follows that for each $k \geqslant 0$ and each $\ell_{k} \leqslant \ell<\ell_{k+1}$,

$$
\text { Leb }\left(\text { tower } n_{\ell}+1\right) \geqslant \operatorname{Leb}\left(\text { tower } n_{\ell}\right)\left(1+\frac{h_{n_{\ell}-k}}{h_{n_{\ell}}}\right) \geqslant\left(1+7^{-k}\right) \text {. }
$$

Therefore it is enough for example to assume that for each $k \geqslant 0$,

$$
\left(1+7^{-k}\right)^{\ell_{k+1}-\ell_{k}} \geqslant 2 \text {. }
$$

Under this assumption, we get at the end a rank-one transformation defined on $\mathbb{R}_{+}$ which preserves the Lebesgue measure.

We will also assume that for each $\ell$,

$$
n_{\ell}>n_{(\ell-1)}+2 \ell \text {. }
$$

\subsection{Construction on a set of sequences}

For technical reasons, it will be more convenient to consider a model of the nearly finite Chacon transformation in which the ambient space is a totally disconnected non compact metric space, and each level of each tower is a compact clopen set.

Consider the countable alphabet $\mathbb{A}:=\{*\} \cup \mathbb{N}$. To each $t \in \mathbb{R}_{+}$, we associate the sequence $\varphi(t)=\left(j_{n}(t)\right)_{n \geqslant 0} \in \mathbb{A}^{\mathbb{N}}$ defined by

$$
j_{n}(t):= \begin{cases}* & \text { if } t \notin \text { tower } n, \\ j & \text { if } t \text { is in level } j \text { of tower } n\left(0 \leqslant j<h_{n}\right) .\end{cases}
$$

By condition (2.1), $\mathbb{R}_{+}=\bigcup_{n}$ tower $n$, and for each $n$, tower $n \subset$ tower $n+1$. Hence for each $t \in \mathbb{R}_{+}$,

$$
\exists n_{0} \geqslant 0: \forall n<n_{0}, j_{n}(t)=* \text {, and } \forall n \geqslant n_{0}, j_{n}(t) \in\left\{0, \ldots, h_{n}-1\right\} .
$$


Step 1

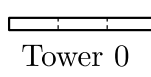

Tower $n$

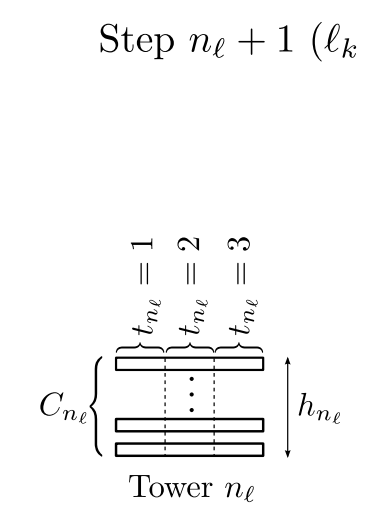

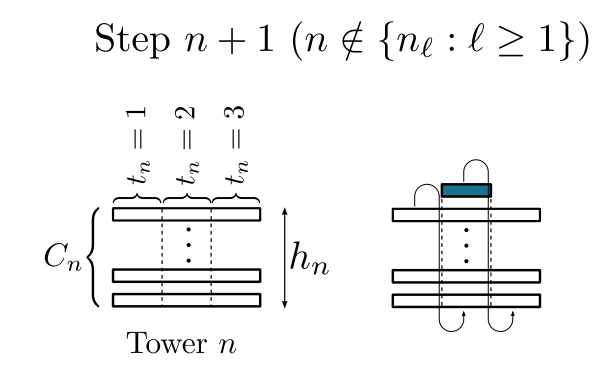

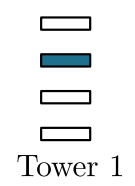

Tower 1
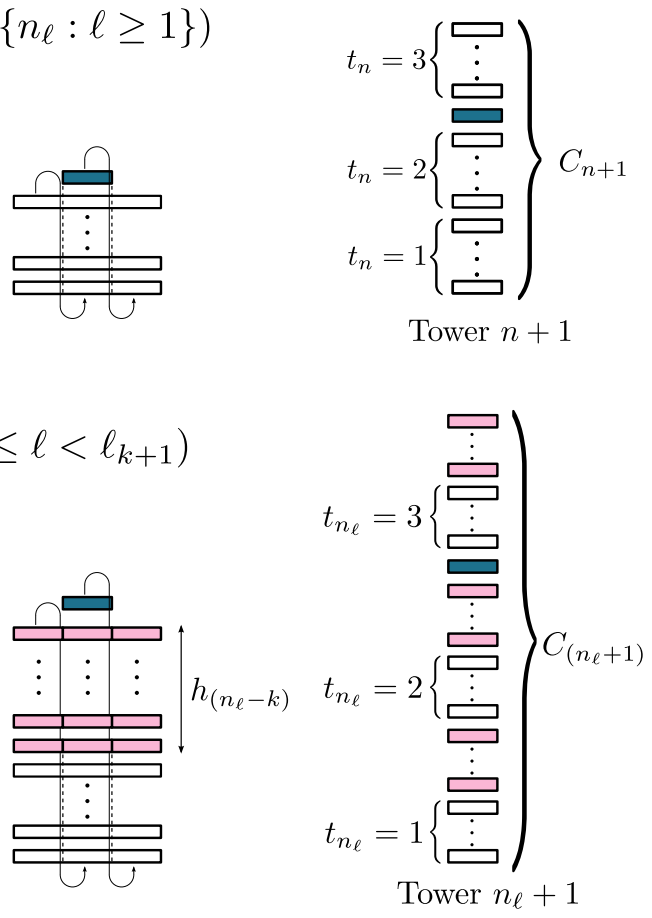

Figure 2.1. Construction of the nearly finite Chacon transformation by cutting and stacking.

Moreover, each level of tower $n+1$ is either completely outside tower $n$ or completely inside a single level of tower $n$. Let us introduce, for each $n \geqslant 1$, the map $p_{n}$ : $\left\{0, \ldots, h_{n+1}-1, *\right\} \rightarrow\left\{0, \ldots, h_{n}-1, *\right\}$ defined by

- $p_{n}(*):=*$,

- $\forall j \in\left\{0, \ldots, h_{n+1}-1\right\}, p_{n}(j):=*$ if level $j$ of tower $n+1$ is completely outside tower $n$, and $p_{n}(j):=j^{\prime} \in\left\{0, \ldots, h_{n}-1\right\}$ if level $j$ of tower $n+1$ is completely inside level $j^{\prime}$ of tower $n$.

Then the sequence $\left(j_{n}(t)\right)_{n \geqslant 0}$ satisfies the following compatibility condition.

$$
\forall n \geqslant 0, j_{n}(t)=p_{n}\left(j_{n+1}(t)\right)
$$

In particular, $j_{n}(t)$ completely determines $j_{m}(t)$ for each $0 \leqslant m \leqslant n$. We also observe that $p_{n}$ satisfies the following property:

(2.3) If $p_{n}(j) \in\left\{0, \ldots, h_{n}-2\right\}$, then $j \in\left\{0, \ldots, h_{n+1}-2\right\}$ and $p_{n}(j+1)=p_{n}(j)+1$.

Now we can define our space $X$, to which belongs $\varphi(t)$ for each $t \in \mathbb{R}_{+}$:

$$
X:=\left\{\left(j_{n}\right)_{n \geqslant 0} \in \mathbb{A}^{\mathbb{N}}: \forall n \geqslant 0, j_{n}=p_{n}\left(j_{n+1}\right) \text { and } \exists n_{0}, j_{n_{0}} \neq *\right\} .
$$


$X$ inherits its topology from the product topology of $\mathbb{A}^{\mathbb{N}}$. In particular it is a totally disconnected metrizable space, but it is not compact (in fact $X$ is not closed in $\mathbb{A}^{\mathbb{N}}$, as the infinite sequence $(*, *, \ldots)$ is in $\bar{X} \backslash X)$.

For each $n \geqslant 0$ and each $x \in X$, we denote by $j_{n}(x)$ the $n$-th coordinate of $x$. For each $j \in\left\{0, \ldots, h_{n}-1\right\}$, we define the subset of $X$

$$
L_{n}^{j}:=\left\{x \in X: j_{n}(x)=j\right\} .
$$

Then $L_{n}^{j}$ is compact and clopen in $X$. Moreover the family of sets $\left(L_{n}^{j}\right)$ form a basis of the topology on $X$.

To define the transformation $T$ on $X$, we need the following easy lemma.

Lemma 2.1. - For each $x=\left(j_{n}\right)_{n \geqslant 0} \in X$, there exists $\bar{n}$ such that, for each $n \geqslant \bar{n}, j_{n} \in\left\{0, \ldots, h_{n}-2\right\}$.

Proof. - Remember that at each step $n_{\ell}+1$, some spacers are added on the last subcolumn of tower $n_{\ell}$. Hence, $j_{n_{\ell}+1}=h_{n_{\ell}+1}-1$ implies $j_{n_{\ell}}=*$. Now take $\ell$ large enough so that $j_{n_{\ell}} \neq *$. Then $j_{n_{\ell}+1}<h_{n_{\ell}+1}-1$, and (2.3) shows by an immediate induction that $j_{m}<h_{m}-1$ for each $m \geqslant n_{\ell}+1$.

We define the measurable transformation $T: X \rightarrow X$ as follows: for $x=\left(j_{n}\right)_{n \geqslant 0} \in$ $X$, we consider the smallest integer $\bar{n}$ satisfying the property given in Lemma 2.1. Then we set $T(x):=\left(j_{n}^{\prime}\right)_{n \geqslant 0}$, where $j_{n}^{\prime}:=j_{n}+1$ if $n \geqslant \bar{n}$, and the finite sequence $\left(j_{1}^{\prime}, j_{2}^{\prime}, \ldots, j_{\bar{n}-1}^{\prime}\right)$ is determined by the value of $j_{\bar{n}}^{\prime}$ and the compatibility conditions $j_{n}^{\prime}=p_{n}\left(j_{n+1}^{\prime}\right), 1 \leqslant n<\bar{n}$. Note that $T$ is one-to-one, and $T(X)=X \backslash\{\boldsymbol{0}\}$, where $\mathbf{0}:=(0,0, \ldots)$.

For each $n \geqslant 1$ and each $0 \leqslant j<h_{n}-1, T\left(L_{n}^{j}\right)=L_{n}^{j+1}$, hence $\left(L_{n}^{0}, \ldots, L_{n}^{h_{n}-1}\right)$ is a Rokhlin tower for $T$. By construction, the family of Rokhlin towers we get in this way has the same structure as the family of Rokhlin towers we constructed by cutting-and-stacking on $\mathbb{R}_{+}$. From now on, "tower $n$ " will rather designate the Rokhlin tower $\left(L_{n}^{0}, \ldots, L_{n}^{h_{n}-1}\right)$ in $X$. The main advantage that we get compared to the construction on $\mathbb{R}_{+}$is the following elementary fact.

Remark 2.2. - If $\left(L_{n}^{j_{n}}\right)_{n \geqslant \bar{n}}$ is a sequence of levels in the successive Rokhlin towers, such that $L_{n+1}^{j_{n+1}}$ is always included in $L_{n}^{j_{n}}$ (equivalently, $j_{n}=p_{n}\left(j_{n+1}\right)$ ), then $\bigcap_{n} L_{n}^{j_{n}}$ is always a singleton

(Note that such an intersection can be empty in the construction on $\mathbb{R}_{+}$.)

Let $\mu$ be the pushforward of the Lebesgue measure on $\mathbb{R}_{+}$by $\varphi$. Then $\mu$ is an infinite, $\sigma$-finite and $T$-invariant measure on $X$, and it satisfies

$$
\forall n \geqslant 0, \forall j \in\left\{0, \ldots, h_{n}-1\right\}, \mu\left(L_{n}^{j}\right)=3^{-n} .
$$

\section{Additional notations}

For each $n \geqslant 0$, we denote by $C_{n}$ the subset of $X$ formed by the union of all the levels of tower $n$. Note that for each $n \geqslant 0, C_{n} \subset C_{n+1}$, and that $X=\cup_{n \geqslant 0} C_{n}$. For $x \in C_{n}$, note that $j_{n}(x)$ indicates the level of tower $n$ to which $x$ belongs. 
We also define a function $t_{n}$ on $C_{n}$, taking values in $\{1,2,3\}$, which indicates for each point whether it belongs to the first, the second, or the third subcolumn of tower $n$. We thus have for $x \in C_{n}$ and $n \notin\left\{n_{\ell}: \ell \geqslant 1\right\}$

$$
j_{n+1}(x)= \begin{cases}j_{n}(x) & \text { if } t_{n}(x)=1, \\ j_{n}(x)+h_{n} & \text { if } t_{n}(x)=2, \\ j_{n}(x)+2 h_{n}+1 & \text { if } t_{n}(x)=3 .\end{cases}
$$

In the case where $n=n_{\ell}$ for some $\ell \geqslant 1$, we have to replace $h_{n}$ by $h_{n_{\ell}}+h_{n_{\ell}-k(\ell)}$ in the above formula. In particular, we always have

$$
j_{n+1}(x) \geqslant j_{n}(x) \text {. }
$$

Consider two integers $0 \leqslant n<m$. By construction, tower $n$ is subdivided into $3^{m-n}$ subcolumns which appear as bundles of $h_{n}$ consecutive levels in tower $m$ : we call them occurrences of tower $n$ inside tower $m$. These occurrences are naturally ordered, from bottom to top of tower $m$. For a point $x$ in tower $n$, the precise occurrence of tower $n$ inside tower $m$ to which $x$ belongs is determined by the sequence $t_{n}(x), t_{n+1}(x), \ldots, t_{m-1}(x)$. For example, $x$ belongs to the last occurrence of tower $n$ inside tower $m$ if and only if $t_{n}(x)=t_{n+1}(x)=\cdots=t_{m-1}(x)=3$.

Remark 2.3. - Observe that for each $\ell \geqslant 2$ and each $n_{(\ell-1)}+1 \leqslant n \leqslant n_{\ell}-1$, there is 0 or 1 spacer between two consecutive occurrences of tower $n$ inside tower $n_{\ell}$.

\subsection{Behaviour of $\mu$-typical points}

Lemma 2.4. - There exists a $\mu$-conull subset $X_{\infty}$ of $X$ satisfying: for each $x \in$ $X_{\infty}$, there exists an integer $\ell(x)$ such that, for all $\ell \geqslant \ell(x)$, for each $n_{(\ell-1)} \leqslant n \leqslant n_{\ell}-\ell$, $x \in C_{n}$ but $x$ is neither in the first hundred nor in the last hundred occurrences of tower $n$ inside tower $n_{\ell}$.

Proof. - If we consider $x$ as a random point chosen according to the normalized $\mu$-measure on $C_{n}$, then the random variables $t_{n}(x), t_{n+1}(x), \ldots, t_{m-1}(x)$ are i.i.d. and uniformly distributed in $\{1,2,3\}$. Hence the probability that $x$ belongs to some specified occurrence of tower $n$ inside tower $m$ is $1 / 3^{m-n}$.

Since the series $\sum 1 / 3^{\ell}$ converges, by Borel Cantelli there exists a subset $X_{n}$ of full $\mu$-measure inside $C_{n}$ such that, for each $x \in X_{n}$, there is only a finite number of integers $\ell$ such that $x$ belongs to the first hundred or to the last hundred occurrences of tower $\left(n_{\ell}-\ell\right)$ inside tower $n_{\ell}$.

Setting

$$
X_{\infty}:=X \backslash \bigcup_{n}\left(C_{n} \backslash X_{n}\right)
$$

we get a conull subset of $X$, and for each $x \in X_{\infty}$, there exists an integer $\ell(x)$ such that, for all $\ell \geqslant \ell(x), x \in C_{n_{(\ell-1)}} \subset C_{n_{\ell}-\ell}$, but $x$ is neither in the first hundred nor in the last hundred occurrence of tower $\left(n_{\ell}-\ell\right)$ inside tower $n_{\ell}$. If $n_{(\ell-1)} \leqslant n \leqslant n_{\ell}-\ell$, the first (respectively last) hundred occurrences of tower $n$ inside tower $n_{\ell}$ are included in the first (respectively last) hundred occurrences of tower $\left(n_{\ell}-\ell\right)$ inside tower $n_{\ell}$, and this concludes the proof. 
Remark 2.5. - In particular, for each $x \in X_{\infty}$, if $n>n_{\ell(x)}$, then $x$ does not belong to the first level of tower $n$. And since $\mathbf{0}$ is in the first level of tower $n$ for each $n$, we have $\mathbf{0} \notin X_{\infty}$.

Remark 2.6. - As $n_{(\ell-1)}+\ell<n_{\ell}-\ell$ by (2.2), we may also assume that for each $x \in X_{\infty}$ and each $\ell \geqslant \ell(x), x$ is neither in the first hundred nor in the last hundred occurrences of tower $n_{(\ell-1)}$ inside tower $n_{\ell}-\ell$.

\section{Invariant Radon measures for Cartesian powers of the nearly finite Chacon transformation}

We fix a natural integer $d \geqslant 1$, and we study the action of the Cartesian power $T^{\times d}$ on $X^{d}$. Recall that a measure $\sigma$ on $X^{d}$ is a Radon measure if it is finite on each compact subset of $X^{d}$ (equivalently, if $\sigma\left(C_{n}^{d}\right)<\infty$ for each $n$ ). In particular, a Radon measure on $X^{d}$ is $\sigma$-finite (but the converse is not true).

Our purpose is to describe all Radon measures on $X^{d}$ which are $T^{\times d}$-invariant and whose marginals are absolutely continuous with respect to $\mu$.

\subsection{Basic facts about Radon measures on $X^{d}$}

We call $n$-box a subset of $C_{n}^{d}$ which is a Cartesian product $L_{n}^{j_{1}} \times \cdots \times L_{n}^{j_{d}}$, where each $L_{n}^{j_{i}}$ is a level of the Rokhlin tower $C_{n}$. A box is a subset which is an $n$-box for some $n \geqslant 0$. The family of all boxes form a basis of compact clopen sets of the topology of $X^{d}$.

We consider the following ring of subsets of $X^{d}$

$$
\mathscr{R}:=\left\{B \subset X^{d}: \exists n \geqslant 0, B \text { is a finite union of } n \text {-boxes }\right\} .
$$

Proposition 3.1. - Any finitely additive functional $\sigma: \mathscr{R} \rightarrow \mathbb{R}_{+}$can be extended to a unique measure on the Borel $\sigma$-algebra $\mathscr{B}\left(X^{d}\right)$, which is Radon.

Proof. - Using Theorems F p. 39 and A p. 54 (Caratheodory's extension theorem) in [Hal50], we only have to prove that, if $\left(R_{k}\right)_{k \geqslant 1}$ is a decreasing sequence in $\mathscr{R}$ such that $\lim _{k \rightarrow \infty} \downarrow \sigma\left(R_{k}\right)>0$, then $\bigcap_{k} R_{k} \neq \emptyset$. But this is obvious since, under this assumption, each $R_{k}$ is a compact nonempty set.

In particular, to define a Radon measure $\sigma$ on $X^{d}$, we only have to define $\sigma(B)$ for each box $B$, with the compatibility condition that, if $B$ is an $n$-box for some $n \geqslant 0$, then $\sigma(B)=\sum_{B^{\prime} \subset B} \sigma\left(B^{\prime}\right)$, where the sum ranges over the $3^{d}(n+1)$-boxes which are contained in $B$.

We call $n$-diagonal a Rokhlin tower for $T^{\times d}$ which is of the form

$$
\left(B, T^{\times d}(B), \ldots,\left(T^{\times d}\right)^{r-1}(B)\right),
$$

where each $\left(T^{\times d}\right)^{j}(B)$ is an $n$-box, and which is maximal in the following sense: $B$ has one projection which is the bottom level $L_{n}^{0}$ of tower $n,\left(T^{\times d}\right)^{r-1} B$ has one projection which is the top level $L_{n}^{h_{n}-1}$ of tower $n$, and the projections of each $\left(T^{\times d}\right)^{j} B, 1 \leqslant j \leqslant r-2$ are neither the bottom level nor the top level of tower $n$. (See Figure 3.1) 


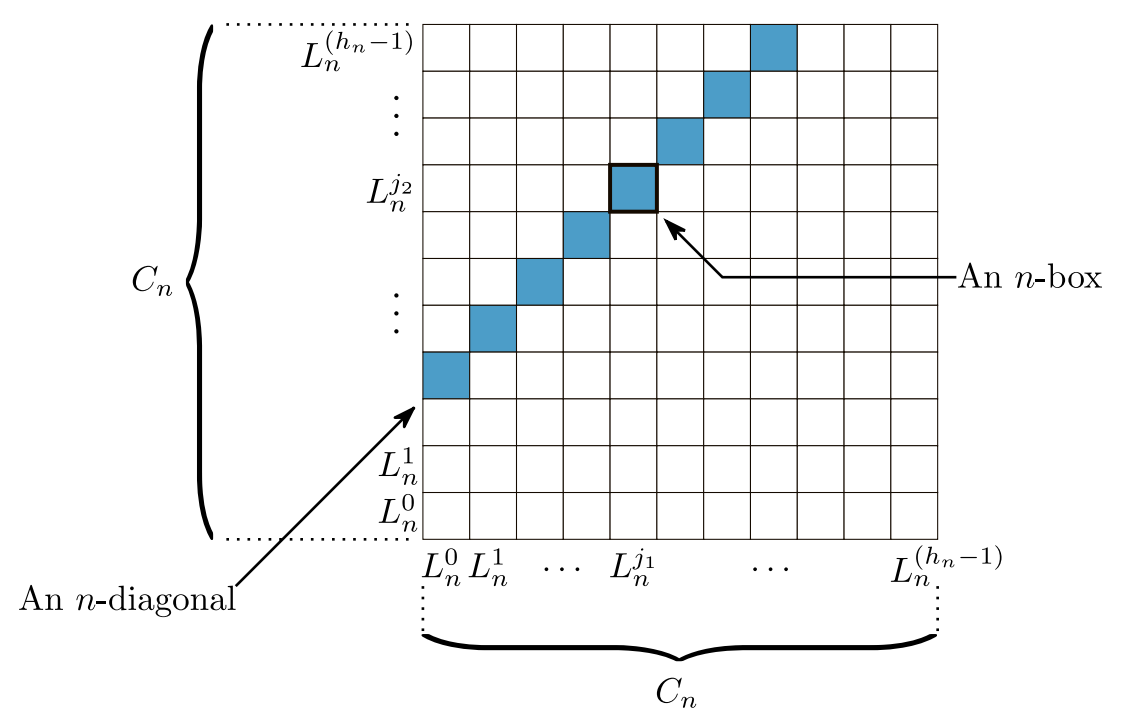

Figure 3.1. An n-diagonal inside $C_{n}^{d}($ here $d=2)$

Lemma 3.2. - Set $X_{\mathbf{0}}^{d}:=\left\{x=\left(x_{1}, \ldots, x_{d}\right) \in X^{d}: \exists i, x_{i}=\mathbf{0}\right\}$. Let $\sigma$ be a Radon measure on $X^{d}$. Then $\sigma$ is $T^{\times d}$-invariant if and only if the following two conditions hold:

(1) $\sigma\left(X_{0}^{d}\right)=0$.

(2) for each $n$, all the $n$-boxes lying on an $n$-diagonal always have the same measure.

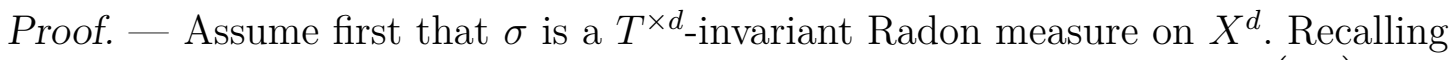
that $\mathbf{0}$ has no preimage by $T$, we see that $\left(T^{\times d}\right)^{-1}\left(X_{\mathbf{0}}^{d}\right)=\emptyset$, whence $\sigma\left(X_{\mathbf{0}}^{d}\right)=0$. Moreover, since $n$-boxes on an $n$-diagonal are levels of a $T^{\times d}$-Rokhlin tower, the second condition obviously holds. Reciprocally, assume that the two conditions given in the statement of the lemma hold. For each $n$, let $\Omega_{n}$ be the subset of $C_{n}^{d}$ constituted of all $n$-boxes of the form $L_{n}^{j_{1}} \times \cdots \times L_{n}^{j_{d}}$, where for each $i j_{i} \neq 0$. Then the second condition implies that $\sigma$ and $\left(T^{\times d}\right)_{*}(\sigma)$ coincide on $\Omega_{n}$ for each $n$. But

$$
\bigcup_{n \geqslant 0} \Omega_{n}=X \backslash X_{0}^{d}
$$

On the other hand, we have $\left(T^{\times d}\right)_{*}(\sigma)\left(X_{\mathbf{0}}^{d}\right)=\sigma\left(\left(T^{\times d}\right)^{-1}\left(X_{\mathbf{0}}^{d}\right)\right)=\sigma(\emptyset)=0$. With the first condition we see that $\sigma$ and $\left(T^{\times d}\right)_{*}(\sigma)$ also coincide on $X_{\mathbf{0}}^{d}$, hence they are equal.

Definition 3.3 (Convergence of Radon measures on $X^{d}$ ). - We say that a sequence $\left(\sigma_{k}\right)$ of Radon measures on $X^{d}$ converges to the nonzero Radon measure $\sigma$ if, for each $n$ large enough so that $\sigma\left(C_{n}^{d}\right)>0$, we have

- $\sigma_{k}\left(C_{n}^{d}\right)>0$ for all large enough $k$,

- for each n-box $B$,

$$
\frac{\sigma_{k}(B)}{\sigma_{k}\left(C_{n}^{d}\right)} \underset{k \rightarrow \infty}{\longrightarrow} \frac{\sigma(B)}{\sigma\left(C_{n}^{d}\right)} .
$$


Observe that, when a sequence of Radon measures converges in the above sense, then its limit is unique up to a multiplicative positive constant. Observe also that the convergence is unchanged if we multiply each measure $\sigma_{k}$ by a positive real number (which may vary with $k$ ).

Remark 3.4. - If the sequence $\left(\sigma_{k}\right)$ of Radon measures on $X^{d}$ converges to the nonzero Radon measure $\sigma$, then for $n$ such that $\sigma\left(C_{n}^{d}\right)>0$ and for each $m \geqslant n$, for each $m$-box $B \subset C_{n}^{d}$, we also have

$$
\frac{\sigma_{k}(B)}{\sigma_{k}\left(C_{n}^{d}\right)} \underset{k \rightarrow \infty}{\longrightarrow} \frac{\sigma(B)}{\sigma\left(C_{n}^{d}\right)}
$$

Consequently, the above holds also when $B \in \mathscr{R}$ is included in $C_{n}^{d}$.

Indeed, as $C_{n}^{d}$ is a finite union of $m$-boxes, we have

$$
\frac{\sigma_{k}\left(C_{n}^{d}\right)}{\sigma_{k}\left(C_{m}^{d}\right)} \underset{k \rightarrow \infty}{\longrightarrow} \frac{\sigma\left(C_{n}^{d}\right)}{\sigma\left(C_{m}^{d}\right)}
$$

Then we can write, for an $m$-box $B \subset C_{n}^{d}$,

$$
\frac{\sigma_{k}(B)}{\sigma_{k}\left(C_{n}^{d}\right)}=\frac{\sigma_{k}(B)}{\sigma_{k}\left(C_{m}^{d}\right)} \frac{\sigma_{k}\left(C_{m}^{d}\right)}{\sigma_{k}\left(C_{n}^{d}\right)} \underset{k \rightarrow \infty}{\longrightarrow} \frac{\sigma(B)}{\sigma\left(C_{m}^{d}\right)} \frac{\sigma\left(C_{m}^{d}\right)}{\sigma\left(C_{n}^{d}\right)}=\frac{\sigma(B)}{\sigma\left(C_{n}^{d}\right)} .
$$

Proposition 3.5. - Let $\left(\sigma_{k}\right)$ be a sequence of Radon measures on $X^{d}$, and assume that there exists some $\underline{n} \geqslant 0$ satisfying

- $\sigma_{k}\left(C_{\underline{n}}^{d}\right)>0$ for all large enough $k$,

- for each $n>\underline{n}$, the sequence $\left(\sigma_{k}\left(C_{n}^{d}\right) / \sigma_{k}\left(C_{\underline{n}}^{d}\right)\right)_{k}$ is bounded.

Then there is a subsequence $\left(k_{j}\right)$ and a nonzero Radon measure $\sigma$ on $X^{d}$ such that $\left(\sigma_{k_{j}}\right)$ converges to $\sigma$.

Proof. - Multiplying each $\sigma_{k}$ by a positive real number if necessary, we may assume that for all large enough $k, \sigma_{k}\left(C_{n}^{d}\right)=1$. Then the second assumption ensures that for each box $B$, the sequence $\left(\sigma_{k}(B)\right)_{k}$ is bounded. By a standard diagonal procedure, we can find a subsequence $\left(k_{j}\right)$ such that for each box $B, \sigma_{k_{j}}(B)$ has a limit which we denote by $\sigma(B)$. Then $\sigma$ defines a finitely additive functional on the ring $\mathscr{R}$ of finite unions of boxes, with values in $\mathbb{R}_{+}$. By Proposition 3.1, $\sigma$ can be extended to a Radon measure on $\mathscr{B}\left(X^{d}\right)$, which is nonzero since $\sigma\left(C_{\underline{n}}^{d}\right)=1$. And we obviously have the convergence of $\left(\sigma_{k_{j}}\right)$ to $\sigma$.

Proposition 3.6. - Let $\left(\sigma_{k}\right)$ and $\left(\gamma_{k}\right)$ be two sequences of Radon measures on $X^{d}$, and assume there exist two nonzero Radon measures $\sigma$ and $\gamma$, an integer $\underline{n} \geqslant 1$ and a real number $\theta>0$ such that

- $\sigma_{k} \underset{k \rightarrow \infty}{\longrightarrow} \sigma$,

- $\gamma_{k} \underset{k \rightarrow \infty}{\stackrel{k \rightarrow \infty}{\longrightarrow}} \gamma$,

- $\forall k, \gamma_{k} \leqslant \sigma_{k}$

- $\forall n \geqslant \underline{n}$, for all large enough $k$ (depending on $n$ ), $\gamma_{k}\left(C_{n}^{d}\right) \geqslant \theta \sigma_{k}\left(C_{n}^{d}\right)$.

Then $\gamma \ll \sigma$. 
Proof. - Let $m \geqslant n \geqslant \underline{n}$, and let $B$ be an $m$-box. For all large enough $k$, we have by assumption

But by Remark 3.4, we have

$$
\frac{\gamma_{k}(B)}{\gamma_{k}\left(C_{n}^{d}\right)} \leqslant \frac{\sigma_{k}(B)}{\theta \sigma_{k}\left(C_{n}^{d}\right)}
$$

$$
\frac{\gamma_{k}(B)}{\gamma_{k}\left(C_{n}^{d}\right)} \underset{k \rightarrow \infty}{\longrightarrow} \frac{\gamma(B)}{\gamma\left(C_{n}^{d}\right)} \text {, and } \frac{\sigma_{k}(B)}{\sigma_{k}\left(C_{n}^{d}\right)} \underset{k \rightarrow \infty}{\longrightarrow} \frac{\sigma(B)}{\sigma\left(C_{n}^{d}\right)}
$$

It follows that

$$
\frac{\gamma(B)}{\gamma\left(C_{n}^{d}\right)} \leqslant \frac{\sigma(B)}{\theta \sigma\left(C_{n}^{d}\right)}
$$

The above inequality extends to each $B \in \mathscr{R}$ contained in $C_{n}^{d}$, and then to each $B \in \mathscr{B}(X)$ contained in $C_{n}^{d}$. In particular, if $B \subset C_{n}^{d}$ is Borel measurable and satisfies $\sigma(B)=0$, then we also have $\gamma(B)=0$. And since $X=\bigcup_{n} C_{n}^{d}$, this concludes the proof.

Remark 3.7. - For each $\underline{\ell} \geqslant 1$, the definition of the ring $\mathscr{R}$ is unchanged if we consider only the finite unions of $n_{\ell}$-boxes, for some $\ell \geqslant \underline{\ell}$. Hence in Propositions 3.5 and 3.6, it is enough for the conclusions to hold that the assumptions be verified only when $n \in\left\{n_{\ell}: \ell \geqslant \underline{\ell}\right\}$.

\subsection{Dissipative case}

Lemma 3.8. - For each $x=\left(x_{1}, \ldots, x_{d}\right) \in X_{\infty}^{d}$, for $\ell>\max \left\{\ell\left(x_{i}\right): i=1, \ldots, d\right\}$, we have

$$
\#\left\{j \geqslant 0:\left(T^{\times d}\right)^{j}(x) \in C_{n_{\ell}+1}^{d}\right\}=\infty .
$$

Proof. - If $\ell>\max \left\{\ell\left(x_{i}\right): i=1, \ldots, d\right\}$, we know by Lemma 2.4 that each coordinate $x_{i}$ is in $C_{n_{\ell}+1}$, but is not in the last occurrence of tower $\left(n_{\ell}+1\right)$ inside tower $n_{(\ell+1)}$. Moreover by Remark 2.5, $x_{i}$ is not in the first level of tower $\left(n_{\ell}+1\right)$. The next occurrence of tower $\left(n_{\ell}+1\right)$ inside tower $n_{(\ell+1)}$ appears after 0 or 1 spacer by Remark 2.3. As the height of tower $\left(n_{\ell}+1\right)$ is $h_{\left(n_{\ell}+1\right)}, T^{h_{\left(n_{\ell}+1\right)}}\left(x_{i}\right)$ is either in the same level of tower $\left(n_{\ell}+1\right)$ as $x_{i}$, or in the level immediately below. Thus $T^{h_{\left(n_{\ell}+1\right)}}\left(x_{i}\right) \in C_{n_{\ell}+1}$. But the same applies to any $\ell^{\prime} \geqslant \ell$, and we get that $T^{h_{\left(n_{\ell^{\prime}}+1\right)}}\left(x_{i}\right)$ is either in the same level of tower $\left(n_{\ell^{\prime}}+1\right)$ as $x_{i}$, or in the level immediately below. Since these two levels are both included in $C_{n_{\ell}+1}$ we get that $T^{h_{\left(n_{\ell^{\prime}}+1\right)}}\left(x_{i}\right) \in C_{n_{\ell}+1}$.

Proposition 3.9. - There is no Radon, $T^{\times d}$-invariant and totally dissipative measure for which $X_{\infty}^{d}$ is a conull set. In particular, there is no Radon, $T^{\times{ }^{d} \text {-invariant }}$ and totally dissipative measure whose marginals are absolutely continuous with respect to $\mu$.

Proof. - Suppose that $\sigma$ is such a measure. Let $W$ be a wandering set for $\sigma$, with

$$
\sigma\left(X^{d} \backslash \bigcup_{j \in \mathbb{Z}}\left(T^{\times d}\right)^{j} W\right)=0 .
$$


As $X_{\infty}^{d}$ is a conull set, we may assume that $W \subset X_{\infty}^{d}$. By the previous lemma, $W=\bigcup_{n} W_{n}$, where

$$
W_{n}:=\left\{x \in W: \#\left\{j \geqslant 0:\left(T^{\times d}\right)^{j}(x) \in C_{n}^{d}\right\}=\infty\right\} .
$$

Hence there exists some $n$ with $\sigma\left(W_{n}\right)>0$. The ergodic decomposition of $\sigma$ writes

$$
\sigma=\int_{W}\left(\sum_{j \in \mathbb{Z}} \delta_{\left(T^{\times d}\right)^{j}(x)}\right) \mathrm{d} \sigma(x),
$$

so we get $\sigma\left(C_{n}^{d}\right)=\infty$, which contradicts the fact that $\sigma$ is Radon.

\subsection{Main result}

An obvious example of a $T^{\times d}$-invariant Radon measure on $X^{d}$ is the product measure $\mu^{\otimes d}$. Another example is what we call a graph measure arising from powers of $T$ : this is a measure $\sigma$ of the form

$$
\sigma\left(A_{1} \times \cdots \times A_{d}\right)=\alpha \mu\left(A_{1} \cap T^{-e_{2}} A_{2} \cap \cdots \cap T^{-e_{d}} A_{d}\right),
$$

for some integers $e_{2}, \ldots, e_{d}$ and some fixed positive real number $\alpha$. Such a measure is concentrated on the subset

$$
\left\{\left(x_{1}, \ldots, x_{d}\right) \in X^{d}: x_{i}=T^{e_{i}} x_{1} \text { for all } i=2, \ldots, d\right\} .
$$

TheOREm 3.10. - For each $d \geqslant 1$, the infinite measure preserving dynamical system $\left(X^{d}, \mu^{\otimes d}, T^{\times d}\right)$ is conservative ergodic.

Moreover, if $\sigma$ is a nonzero, Radon, $T^{\times d}$-invariant and ergodic measure on $X^{d}$, such that

$$
\sigma\left(X^{d} \backslash X_{\infty}^{d}\right)=0
$$

then there exists a partition of $\{1, \ldots, d\}$ into $r$ subsets $D_{1}, \ldots, D_{r}$, such that $\sigma=$ $\sigma^{D_{1}} \otimes \cdots \otimes \sigma^{D_{r}}$, where $\sigma^{D_{j}}$ is a graph measure on $X^{D_{j}}$ arising from powers of $T$.

Corollary 3.11. - If $\sigma$ is a nonzero, Radon, $T^{\times d}$-invariant measure on $X^{d}$, whose marginals are absolutely continuous with respect to $\mu$, then $\sigma$ decomposes as a sum of countably many ergodic components, which are all of the form described in Theorem 3.10.

To prove Theorem 3.10 in the case $d=1$, we even do not need assumption (3.2) as we can show that $\mu$ is, up to a multiplicative constant, the only $T$-invariant, Radon measure on $X$ (the proof is the same as for the Chacon infinite transformation, see [JRdlR18, Proposition 2.4]).

We also note that, if we have proved the second part of the theorem for some $d \geqslant 2$, then the first one follows immediately. Indeed, if $\mu^{\otimes d}$ were not ergodic, then almost all its ergodic components would satisfy (3.2), hence would be a product of graph measures different from $\mu^{\otimes d}$. But this would mean that for $\mu^{\otimes d}$-almost all $x \in X^{d}$, there exist at least two coordinates of $x$ lying on the same $T$-orbit, which of course is absurd. Hence $\mu^{\otimes d}$ is ergodic, and by Proposition 3.9, it is conservative. 
The remainder of this paper is devoted to the proof by induction of the second part of Theorem 3.10. So we now assume that for some $d \geqslant 2$, the statement is true up to $d-1$. We consider a nonzero, Radon, $T^{\times d}$-invariant and ergodic measure $\sigma$ on $X^{d}$, satisfying (3.2).

We will show that either $\sigma$ is a graph measure arising from powers of $T$, or it can be decomposed into a product of two measures $\sigma_{1} \times \sigma_{2}, \sigma_{i}$ being a $T^{\times d_{i} \text {-invariant }}$ Radon measure on $X^{d_{i}}$ for some $1 \leqslant d_{i}<d, d_{1}+d_{2}=d$. In this latter case we can apply the induction hypothesis to each $\sigma_{i}$, which yields the announced result.

\subsection{Choice of a $\sigma$-typical point}

By Proposition 3.9, the system $\left(X^{d}, \sigma, T^{\times d}\right)$ is conservative ergodic. By Hopf's ergodic theorem, if $B \subset C \subset X^{d}$ with $0<\sigma(C)<\infty$, we have for $\sigma$-almost every point $x=\left(x_{1}, \ldots, x_{d}\right) \in X^{d}$

$$
\frac{\sum_{j \in J} \mathbb{1}_{B}\left(\left(T^{\times d}\right)^{j} x\right)}{\sum_{j \in J} \mathbb{1}_{C}\left(\left(T^{\times d}\right)^{j} x\right)} \underset{|J| \rightarrow \infty}{\longrightarrow} \frac{\sigma(B)}{\sigma(C)},
$$

where the sums in the above expression range over a set $J$ of consecutive integers containing 0 .

We say that $x \in X^{d}$ is typical if, for all $n$ large enough so that $\sigma\left(C_{n}^{d}\right)>0$, Property (3.3) holds whenever $B$ is an $n$-box and $C$ is $C_{n}^{d}$. We know that $\sigma$-almost every $x \in X^{d}$ is typical. Therefore, there exists a point $x=\left(x_{1}, \ldots, x_{d}\right)$ such that

(3.4) For each $j \in \mathbb{Z},\left(T^{\times d}\right)^{j} x$ is typical.

Since there are only countably many boxes, we may also assume that

(3.5) For each box $B, x \in B \Longrightarrow \sigma(B)>0$.

Moreover, by (3.2), we can further assume that

(3.6) $\forall i=1, \ldots, d, \quad x_{i} \in X_{\infty}$.

From now on, we fix a point $x=\left(x_{1}, \ldots, x_{d}\right)$ satisfying the above assumptions (3.4), (3.5) and (3.6). We will derive properties of $\sigma$ from the observations made on the orbit of this point $x$.

By an interval, we mean in this paper a finite set of consecutive integers. We will need the following key notion in our argument.

Definition 3.12. - We call $n$-crossing a maximal interval $J \subset \mathbb{Z}$ with the following properties:

- $\left(T^{\times d}\right)^{j} x \in C_{n}^{d}$ for each $j \in J$,

- for each $1 \leqslant i \leqslant d, j \mapsto t_{n}\left(T^{j} x_{i}\right)$ is constant on $J$.

An $n$-crossing is said to be synchronized if $t_{n}\left(T^{j} x_{1}\right)=\cdots=t_{n}\left(T^{j} x_{d}\right)$ for each $j$ in this $n$-crossing.

Note that an $n$-crossing has at most $h_{n}$ elements. If $j$ is the smallest (respectively the largest) element of an $n$-crossing, then there exists $1 \leqslant i \leqslant d$ such that $T^{j} x_{i}$ is in the first (respectively the last) level of tower $n$. Observe also that when $j$ runs over an $n$-crossing, $\left(T^{\times d}\right)^{j} x$ successively passes through each $n$-box of some $n$-diagonal. 


\subsection{Characterizations of graph measures arising from powers of $T$}

LEMmA 3.13. - The following assertions are equivalent:

(1) $\sigma$ is a graph measure arising from powers of $T$;

(2) $\exists e_{2}, \ldots, e_{d} \in \mathbb{Z}: x_{i}=T^{e_{i}} x_{1}$ for each $i=2, \ldots, d$;

(3) $\exists \underline{n}: \forall n \geqslant \underline{n}, t_{n}\left(x_{1}\right)=\cdots=t_{n}\left(x_{d}\right)$;

(4) $\exists j, \exists \underline{n}: \forall n \geqslant \underline{n}, t_{n}\left(T^{j} x_{1}\right)=\cdots=t_{n}\left(T^{j} x_{d}\right)$.

Proof. - Let us first prove that (1) $\Longrightarrow(2)$. If $\sigma$ is a graph measure arising from powers of $T$, then there exist a positive real number $\alpha$ and integers $e_{2}, \ldots, e_{d}$ such that for all measurable subsets $A_{1}, \ldots, A_{d}$ of $X,(3.1)$ holds. Observe that, if $\ell$ is large enough so that $h_{n_{\ell}-k(\ell)}>\max \left\{\left|e_{2}\right|, \ldots,\left|e_{d}\right|\right\}$, then for each $i=2, \ldots, d$ and each $j, j^{\prime} \in\left\{0, \ldots, h_{n_{\ell}}-1\right\}$,

$$
L_{n_{\ell}}^{j} \cap T^{-e_{i}} L_{n_{\ell}}^{j^{\prime}}= \begin{cases}L_{n_{\ell}}^{j} & \text { if } j^{\prime}=j+e_{i}, \\ \emptyset & \text { otherwise. }\end{cases}
$$

It follows that the only $n_{\ell}$-boxes that may be charged by $\sigma$ are of the form $L_{n_{\ell}}^{j_{1}} \times$ $L_{n_{\ell}}^{j_{1}+e_{2}} \times \cdots \times L_{n_{\ell}}^{j_{1}+e_{d}}$ for some $j_{1}$. By assumption (3.5), it follows that for each $i=2, \ldots, d, j_{n_{\ell}}\left(x_{i}\right)=j_{n_{\ell}}\left(x_{1}\right)+e_{i}$. Since this is true for all large enough $\ell$, this in turn implies that for each $i=2, \ldots, d, x_{i}=T^{e_{i}} x_{1}$.

Conversely, if (2) holds, the same argument shows that if $\ell$ is large enough so that $h_{n_{\ell}-k(\ell)}>\max \left\{\left|e_{2}\right|, \ldots,\left|e_{d}\right|\right\}$, then the only $n_{\ell^{-}}$boxes that can contain $x$ are the $n_{\ell}$-boxes of the form $L_{n_{\ell}}^{j_{1}} \times L_{n_{\ell}}^{j_{1}+e_{2}} \times \cdots \times L_{n_{\ell}}^{j_{1}+e_{d}}$ for some $j_{1}$. Note that the $n_{\ell^{-}}$boxes of this form constitute an $n_{\ell}$-diagonal, which we denote by $D$. But (2) is also valid for each $\left(T^{\times d}\right)^{j} x, j \in \mathbb{Z}$ hence the argument also applies to each $\left(T^{\times d}\right)^{j} x$. Thus, if $B$ is an $n_{\ell}$-box which is not on $D$, then $\left(T^{\times d}\right)^{j} x \notin B$ for each $j \in \mathbb{Z}$. Now, remembering that $x$ is typical for $\sigma$, we have for each $n_{\ell}$-box $B=L_{n_{\ell}}^{j_{1}} \times \cdots \times L_{n_{\ell}}^{j_{d}}$

$$
\frac{\sigma(B)}{\sigma\left(C_{n_{\ell}}^{d}\right)}=\lim _{k \rightarrow \infty} \frac{\sum_{-k \leqslant j \leqslant k} \mathbb{1}_{B}\left(\left(T^{\times d}\right)^{j} x\right)}{\sum_{-k \leqslant j \leqslant k} \mathbb{1}_{C_{n_{\ell}}}\left(\left(T^{\times d}\right)^{j} x\right)} .
$$

The above limit is 0 if $B$ is not on $D$. Moreover, note that each time the orbit of $x$ passes through $C_{n_{\ell}}^{d},\left(T^{\times d}\right)^{j} x$ successively passes through each $n_{\ell}$-box on $D$. Hence if $B$ is on $D$, the limit is equal to the inverse of the number of $n_{\ell}$ boxes on $D$. In particular the limit is proportional to $\mu\left(L_{n_{\ell}}^{j_{1}} \cap T^{-e_{2}} L_{n_{\ell}}^{j_{1}} \cap \cdots \cap T^{-e_{d}} L_{n_{\ell}}^{j_{d}}\right)$. The coefficient of proportionality depends a priori on $\ell$, but since each $n_{\ell^{-}}$box is a union of disjoint $n_{\ell+1}$-boxes, we see that in fact this coefficient does not depend on $\ell$. Finally, this gives (3.1) in the case of an $n_{\ell}$-box for each large enough $\ell$, and this is enough to conclude that (3.1) holds for each measurable set of the form $A_{1} \times \cdots \times A_{d}$. We have so far proved the equivalence of (1) and (2).

Now let us turn to the proof of $(2) \Longrightarrow(3)$. Since $x_{1} \in X_{\infty}$, we have $j_{n}\left(x_{1}\right) \rightarrow \infty$ and $h_{n}-j_{n}\left(x_{1}\right) \rightarrow \infty$ as $n \rightarrow \infty$. If (2) holds, we then have $j_{n}\left(x_{i}\right)=j_{n}\left(x_{1}\right)+e_{i}$ for each $i=1, \ldots, d$ and each $n$ large enough so that $\min \left\{j_{n}\left(x_{1}\right), h_{n}-j_{n}\left(x_{1}\right)\right\}>$ $\max \left\{\left|e_{2}\right|, \ldots,\left|e_{d}\right|\right\}$. But then for such an $n$ we also have $t_{n}\left(x_{i}\right)=t_{n}\left(x_{1}\right)$ for each $i=1, \ldots, d$.

The implication $(3) \Longrightarrow(4)$ is obvious. 
Assume now that (4) holds with $j=0$ (i.e. that, in fact, (3) holds). For $i=2, \ldots, d$, we then have by an easy induction that $j_{n}\left(x_{i}\right)-j_{n}\left(x_{1}\right)=j_{\underline{n}}\left(x_{i}\right)-j_{\underline{n}}\left(x_{1}\right)$ for each $n \geqslant \underline{n}$. Setting $e_{i}:=j_{n}\left(x_{i}\right)-j_{n}\left(x_{1}\right)$ for $i=2, \ldots, d$, we get that $x_{i}=T^{e_{i}} x_{1}$ and we have (2). Now if (4) holds with some $j \in \mathbb{Z}$, we get $(2)$ for $\left(T^{\times d}\right)^{j} x$, which is clearly equivalent to (2) for $x$. Thus we have proved that $(4) \Longrightarrow(2)$ and this concludes the proof of the lemma.

For the remainder of the paper, we also fix a real number $0<\eta<1$, small enough so that $\eta<\frac{1}{100 d}$. In particular we will need the inequality $(1-\eta)^{2}>1 / 2$.

Definition 3.14. - For each $n$, let $\boldsymbol{I}_{n}:=\left\{-\left\lfloor h_{n} / 2\right\rfloor, \ldots,-\left\lfloor h_{n} / 2\right\rfloor+h_{n}-1\right\}$ be the interval of length $h_{n}$ and centered at 0 . For each $n \geqslant 0$, we call substantial $n$-crossing any $n$-crossing whose intersection with $\boldsymbol{I}_{n}$ contains at least $\eta h_{n}$ elements.

LEMmA 3.15. - If $n=n_{(\ell-1)}$ for some large enough $\ell$, then substantial $n$ crossings cover a proportion at least $(1-(d+2) \eta)$ of $\boldsymbol{I}_{n}$. In particular, there exists at least one substantial $n$-crossing. Moreover, if all substantial $n$-crossings are synchronized, then each substantial $n$-crossing is of size at least $(1-(d+2) \eta) h_{n}$, and there are at most two of them.

Proof. - Let us start by considering the case of an integer $n$ which is of the form $n=n_{(\ell-1)}$ for some $\ell>\max _{i} \ell\left(x_{i}\right)$. We also assume that $\ell$ is large enough so that

$$
\frac{1}{3^{k(\ell-1)}}<\frac{\eta}{2 d}
$$

We set $n^{\prime}:=n_{(\ell-1)}-k(\ell-1)$, and we observe that the above assumption ensures that

$$
\frac{h_{n^{\prime}}+1}{h_{n}}<\frac{\eta}{d}
$$

We know by Lemma 2.4 that $x \in C_{n_{(\ell-1)}}^{d}=C_{n}^{d}$, and that the interval

$$
\left\{-100 h_{\left(n_{\ell}-\ell\right)}, \ldots, 100 h_{\left(n_{\ell}-\ell\right)}\right\}
$$

is contained in a single $n_{\ell^{-}}$crossing. A fortiori, $\boldsymbol{I}_{n}$ is contained in a single $n_{\ell^{-}}$crossing. Therefore, if a coordinate $T^{j} x_{i}$ reaches the top of tower $n$ and comes back to $C_{n}$ on the interval $\boldsymbol{I}_{n}$, then the two passages in $C_{n}$ are separated by at most $h_{n^{\prime}}+1$. Moreover, this can happen at most once on the interval $\boldsymbol{I}_{n}$ for each $i$. It follows that the set of integers $j \in \boldsymbol{I}_{n}$ such that $\left(T^{\times d}\right)^{j} x \notin C_{n}^{d}$ is constituted of at most $d$ pieces, and its cardinality is bounded above by $\eta h_{n}$ by (3.7). Then there exist at most $(d+1) n$-crossings intersecting $\boldsymbol{I}_{n}$, and they cover a proportion at least $(1-\eta)$ of $\boldsymbol{I}_{n}$. Now the proportion of $\boldsymbol{I}_{n}$ covered by $n$-crossings which are not substantial is less than $(d+1) \eta$, hence the proportion of $\boldsymbol{I}_{n}$ covered by substantial $n$-crossings is at least $(1-(d+2) \eta)$. This proves the first part of the lemma

Let us assume now that all substantial $n$-crossings are synchronized. If we have only one substantial $n$-crossing, then this $n$-crossing is of size at least $(1-(d+2) \eta) h_{n}$, and we have for $j$ in this $n$-crossing

$$
\left|j_{n}\left(T^{j} x_{i_{1}}\right)-j_{n}\left(T^{j} x_{i_{2}}\right)\right| \leqslant(d+2) \eta h_{n} .
$$


If we have at least two substantial $n$-crossings, note that between two of them, there is at least one coordinate passing through the top of tower $n$, and for which $t_{n}$ has increased by $1 \bmod 3$. Since the $t_{n}\left(T^{j} x_{i}\right), i=1, \ldots, d$ are supposed to be equal on each substantial $n$-crossing, we deduce that each coordinate passes through the top of tower $n$ between two substantial $n$-crossings. As this happens at most once for each coordinate on $\boldsymbol{I}_{n}$, we see that there are at most two substantial $n$-crossings. Finally, from the first part of the lemma it follows that two consecutive substantial $n$-crossings are separated by at most $(d+2) \eta h_{n}$ points. We deduce that, on any substantial $n$-crossing, (3.8) holds, hence each substantial $n$-crossing is of size at least $(1-(d+2) \eta) h_{n}$.

Remark 3.16. - The preceding lemma extends easily to the case when $n_{(\ell-1)} \leqslant$ $n \leqslant n_{\ell}-\ell$. Indeed, when $n_{(\ell-1)}+1 \leqslant n \leqslant n_{\ell}-\ell$ the proof is even simpler, as two successive passages in $C_{n}$ are now separated by at most one.

Proposition 3.17. - The measure $\sigma$ is a graph measure arising from powers of $T$ if and only if for each large enough $n$, all substantial $n$-crossings are synchronized.

Proof. - First assume that $\sigma$ is a graph measure arising from powers of $T$. Then by Lemma 3.13, we know that there exists $e_{2}, \ldots, e_{d} \in \mathbb{Z}$ such that $x_{i}=T^{e_{i}} x_{1}$ for each $i=2, \ldots, d$. Take $n$ large enough so that $\max \left\{\left|e_{2}\right|, \ldots,\left|e_{d}\right|\right\}<\eta h_{n}$. Let $J$ be a substantial $n$-crossing. In particular the size of $J$ is at least $\eta h_{n}$. Hence there exists $\bar{j} \in J$ such that $\eta h_{n} \leqslant j_{n}\left(T^{\bar{j}} x_{1}\right) \leqslant(1-\eta) h_{n}$. We deduce that $j_{n}\left(T^{\bar{j}} x_{i}\right)=j_{n}\left(T^{\bar{j}} x_{1}\right)+e_{i}$ for each $i=2, \ldots, d$. But we also have $\eta h_{n} \leqslant j_{n+1}\left(T^{\bar{j}} x_{1}\right) \leqslant(1-\eta) h_{n}$ and this ensures that $j_{n+1}\left(T^{\bar{j}} x_{i}\right)=j_{n+1}\left(T^{\bar{j}} x_{1}\right)+e_{i}$. By $(2.4)$, the equality $j_{n+1}\left(T^{\bar{j}} x_{i}\right)-j_{n+1}\left(T^{\bar{j}} x_{1}\right)=$ $j_{n}\left(T^{\bar{j}} x_{i}\right)-j_{n}\left(T^{\bar{j}} x_{1}\right)$ implies $t_{n}\left(T^{\bar{j}} x_{i}\right)=t_{n}\left(T^{\bar{j}} x_{1}\right)$. Finally, as $j \mapsto t_{n}\left(T^{j} x_{i}\right)$ is constant on the $n$-crossing $J$, we see that $J$ is synchronized.

Conversely, assume that there exists $\underline{n}$ such that for each $n \geqslant \underline{n}$, all substantial $n$-crossings are synchronized. Without loss of generality, we may assume that $\underline{n}$ is of the form $n_{(\ell-1)}$, for some $\ell$ large enough to apply Lemma 3.15. Then we know that there exists at least one substantial $\underline{n}$-crossing $J_{\underline{n}}$, of size at least $(1-(d+2) \eta) h_{\underline{n}}$. For $j \in J_{\underline{n}}$ and for each $i=2, \ldots, d,\left|j_{\underline{n}}\left(T^{j} x_{i}\right)-j_{\underline{n}}\left(T^{j} x_{1}\right)\right| \leqslant(d+2) \eta h_{\underline{n}}$. Let us prove by induction that for each $n \geqslant \underline{n}$, there exists a substantial $n$-crossing $J_{n}$, of size at least $(1-(d+2) \eta) h_{n}$, and containing $J_{\underline{n}}$. We already know that this property is true for $\underline{n}$. Assume it is true up to $n$ for some $n \geqslant \underline{n}$. Then, the $n$-crossing $J_{n}$ extends to a unique $(n+1)$-crossing $J_{n+1}$. As $J_{n}$ intersects $\boldsymbol{I}_{n}$ and is of size at most $h_{n}, J_{n} \subset \boldsymbol{I}_{n+1}$. It follows that

$$
\left|J_{n+1} \cap \boldsymbol{I}_{n+1}\right| \geqslant\left|J_{n}\right| \geqslant(1-(d+2) \eta) h_{n} \geqslant \eta h_{n+1},
$$

which proves that $J_{n+1}$ is a substantial $(n+1)$-crossing. Moreover, since the size of $J_{n}$ is at least $(1-(d+2) \eta) h_{n}$, we have for $j \in J_{n}$ and each $i=2, \ldots, d \mid j_{n}\left(T^{j} x_{i}\right)-$ $j_{n}\left(T^{j} x_{1}\right) \mid \leqslant(d+2) \eta h_{n}$. But $J_{n}$ is synchronized, hence by (2.4), we have for $j \in J_{n}$

$$
\left|j_{n+1}\left(T^{j} x_{i}\right)-j_{n+1}\left(T^{j} x_{1}\right)\right|=\left|j_{n}\left(T^{j} x_{i}\right)-j_{n}\left(T^{j} x_{1}\right)\right| \leqslant(d+2) \eta h_{n} \leqslant(d+2) \eta h_{n+1} .
$$

This equality extends to $j \in J_{n+1}$ since the difference is constant on an $(n+1)$ crossing. This proves that the size of $J_{n+1}$ is at least $(1-(d+2) \eta) h_{n+1}$. Now if we take any $j \in J_{\underline{n}}$, we have $j \in J_{n}$ for each $n \geqslant \underline{n}$, and since we assumed that 
each substantial $n$-crossing is synchronized, we have $t_{n}\left(T^{j} x_{1}\right)=\cdots=t_{n}\left(T^{j} x_{d}\right)$, i.e. we have (4) of Lemma 3.13. This proves that $\sigma$ is a graph measure arising from powers of $T$.

Remark 3.18. - In the preceding proof, the induction provides in fact a stronger inequality for the sizes of the substantial $n$-crossings $\left(J_{n}\right):\left|J_{n}\right| \geqslant h_{n}-(d+2) \eta h_{\underline{n}}$.

\section{Combinatorics of some sets of integers}

The purpose of this section is to establish Proposition 4.1 on the combinatorics of the set of integers $j$ such that $\left(T^{\times d}\right)^{j} x \in C_{n}^{d}$ for a given large $n$.

Proposition 4.1. - There exist constants $K_{1}>0$ and $K_{2}>0$ such that, for any large enough integer $\underline{\ell}$, and any integer $1 \leqslant c \leqslant h_{n_{\ell}}$, the following holds: if $I \subset \mathbb{Z}$ is an interval contained in an $n_{(\ell+\ell)}$-crossing for some $\bar{\ell} \geqslant 1$, and if the length of $I$ is at least $\eta h_{n_{(\underline{\ell}+\ell-1)}}$, then

- the proportion of integers $j \in I$ such that $\left(T^{\times d}\right)^{j} x \in C_{n_{\ell}}^{d}$ is at least $(1-\eta)^{2 \ell}$;

- among all the integers $j \in I$ such that $\left(T^{\times d}\right)^{j} x \in C_{n_{\ell}}^{d}$, the proportion of those belonging to an $n_{\underline{\ell}}$-crossing of size $\leqslant c$ is bounded above by

$$
K_{1} \frac{c}{h_{n_{\underline{\ell}}}}+\frac{K_{2}}{3 \underline{\underline{\ell}}} .
$$

For this we will introduce a hierarchy of more and more complex subsets of $\mathbb{Z}$, prove by induction some combinatorial results on abstract sets in this hierarchy, and finally show how to apply these results in the particular case we are interested in.

\subsection{A hierarchy of subsets of $\mathbb{Z}$}

This part of the argument is completely abstract and independent of the rest of the paper, but we keep the notations $d$ (an integer, $d \geqslant 2$ ) and $\eta$ (a positive real number between 0 and 1 ). We set

$$
K_{1}:=\frac{1+\frac{2}{\eta}}{1-\eta} d
$$

We fix two sequences of positive integers $\left(c_{\ell}\right)_{\ell \geqslant 1}$ and $\left(s_{\ell}\right)_{\ell \geqslant 1}$, satisfying

$$
\forall \ell \geqslant 1, \frac{s_{\ell}}{c_{\ell}}<\frac{1}{d} \frac{\eta}{\eta+1} \eta
$$

and

$$
\forall \ell \geqslant 1, \frac{c_{\ell}}{c_{\ell+1}}<\frac{\eta}{K_{1}} .
$$

Let $F \subset \mathbb{Z}$, and let $I \subset \mathbb{Z}$ be an interval. We call piece of $F \cap I$ any maximal interval included in $F \cap I$, and we call hole of $F \cap I$ any maximal interval included in $I \backslash F$. (Thus, $I$ is the disjoint union of the pieces and the holes of $F \cap I$, which alternate.) 


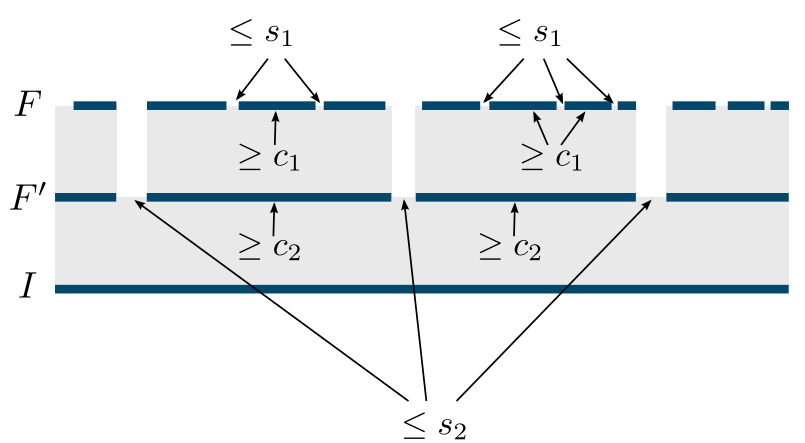

Figure 4.1. A set $F$ of order 2 inside an interval $I$.

We say that $F$ is of order 1 inside the interval $I$ if

- each hole of $F \cap I$ is of size $\leqslant s_{1}$,

- two consecutive holes of $F \cap I$ are always separated by a piece of size at least $c_{1}$.

Recursively, we say that $F$ is of order $\ell \geqslant 2$ inside the interval $I$ if there exists a subset $F^{\prime} \subset \mathbb{Z}$ such that

- $F \subset F^{\prime}$

- each hole of $F^{\prime} \cap I$ is of size $\leqslant s_{\ell}$,

- two consecutive holes of $F^{\prime} \cap I$ are always separated by a piece of size at least $c_{\ell}$

- for each piece $I^{\prime}$ of $F^{\prime} \cap I, F$ is of order $(\ell-1)$ inside $I^{\prime}$.

(See Figure 4.1.) Note that, if $F$ is of order $\ell$ inside the interval $I$, then $F$ is of order $\ell$ inside each subinterval $J \subset I$.

Lemma 4.2. - Let $F_{1}, \ldots, F_{d}$ be $d$ subsets of $\mathbb{Z}$, and let $I \subset \mathbb{Z}$ be an interval. Assume that for some $\ell \geqslant 1, F_{i}$ is of order $\ell$ inside $I$ for each $i=1, \ldots, d$, and that the size of $I$ is at least $\eta c_{\ell}$. Set $F:=\bigcap_{i=1}^{d} F_{i}$. Then

- the density of $F$ inside $I$ satisfies

$$
\frac{|F \cap I|}{|I|} \geqslant(1-\eta)^{2 \ell},
$$

- for a given integer $c, 1 \leqslant c<h_{1}$, the proportion of integers in $F \cap I$ lying in pieces of $F \cap I$ with size $\leqslant c$ is bounded above by

$$
K_{1}\left(\frac{c}{c_{1}}+\frac{c_{1}}{c_{2}} \frac{1}{(1-\eta)^{4}}+\cdots+\frac{c_{\ell-1}}{c_{\ell}} \frac{1}{(1-\eta)^{2 \ell}}\right)
$$

Proof. - Let us first establish the result for $\ell=1$. We assume that each $F_{i}$ is of order 1 inside $I$, and that $|I| \geqslant \eta c_{1}$. For each $i=1, \ldots, d$, let $k_{i}$ be the number of holes of $F_{i} \cap I$. Then by definition of order 1 , there are at least $k_{i}-1$ pieces of $F_{i} \cap I$ with size at least $c_{1}$, whence $c_{1}\left(k_{i}-1\right) \leqslant|I|$, and

$$
k_{i} \leqslant|I| / c_{1}+1 \text {. }
$$


Since each hole of $F_{i} \cap I$ has size $\leqslant s_{1}$, we deduce that the cardinality of $I \backslash F_{i}$ is bounded by $s_{1}\left(|I| / c_{1}+1\right)$. This yields by the inequality $1 \leqslant \frac{1}{\eta} \frac{|I|}{c_{1}}$ and (4.1):

$$
|I \backslash F| \leqslant d s_{1}\left(|I| / c_{1}+1\right) \leqslant d\left(1+\frac{1}{\eta}\right) s_{1}|I| / c_{1} \leqslant \eta|I| .
$$

We thus get $|F \cap I| /|I| \geqslant 1-\eta \geqslant(1-\eta)^{2}$, which is the first point. Moreover, the number $k$ of holes of $F \cap I$ satisfies $k \leqslant k_{1}+\cdots+k_{d} \leqslant d|I| / c_{1}+d$, whence the number $m$ of pieces of $F \cap I$ satisfies

$$
m \leqslant d|I| / c_{1}+d+1 \leqslant d|I| / c_{1}+2 d \leqslant d\left(1+\frac{2}{\eta}\right)|I| / c_{1} .
$$

It follows that the number $r$ of points of $F \cap I$ lying in a piece of size $\leqslant c$ satisfies

$$
r \leqslant m c \leqslant d\left(1+\frac{2}{\eta}\right)|I| \frac{c}{c_{1}} .
$$

As we already know that $|F \cap I| \geqslant(1-\eta)|I|$, we get by definition of $K_{1}$

$$
\frac{r}{|F \cap I|} \leqslant \frac{\left(1+\frac{2}{\eta}\right)}{1-\eta} d \frac{c}{c_{1}}=K_{1} \frac{c}{c_{1}},
$$

which establishes the second point for $\ell=1$.

Now we assume by induction that the result is true up to $\ell-1$ for some $\ell \geqslant 2$ and we consider a family $\left(F_{i}\right)_{1 \leqslant i \leqslant d}$ of subsets of $\mathbb{Z}$, which are of order $\ell$ inside an interval $I$ satisfying $|I| \geqslant \eta c_{\ell}$. By definition of order $\ell$, for each $i$ there exists a subset $F_{i}^{\prime} \subset \mathbb{Z}$ satisfying

- $F_{i} \subset F_{i}^{\prime}$,

- each hole of $F_{i}^{\prime} \cap I$ is of size $\leqslant s_{\ell}$,

- two consecutive holes of $F_{i}^{\prime} \cap I$ are always separated by a piece of size at least $c_{\ell}$

- for each piece $I^{\prime}$ of $F_{i}^{\prime} \cap I, F_{i}^{\prime}$ is of order $(\ell-1)$ inside $I^{\prime}$.

Since $|I| \geqslant \eta c_{\ell}$, the argument developed for order 1 applies for $F^{\prime}:=\bigcap_{i=1}^{d} F_{i}^{\prime}$ (with $\left(c_{\ell-1}, c_{\ell}, s_{\ell}\right)$ in place of $\left.\left(c, c_{1}, s_{1}\right)\right)$. We thus get

$$
\left|F^{\prime} \cap I\right| \geqslant(1-\eta)|I|,
$$

and denoting by $r^{\prime}$ the number of points of $F^{\prime} \cap I$ lying in pieces of $F^{\prime} \cap I$ of size $<c_{\ell-1}$, we have (using also (4.2))

$$
\frac{r^{\prime}}{\left|F^{\prime} \cap I\right|} \leqslant K_{1} \frac{c_{\ell-1}}{c_{\ell}}<\eta
$$

Let $G$ stand for the union of all pieces of $F^{\prime} \cap I$ of size $\geqslant c_{\ell-1}$. The above inequality can be rewritten as

$$
\frac{|G|}{\left|F^{\prime} \cap I\right|}>(1-\eta)
$$


Let $J$ be an arbitrary piece of $G$. Since for each $i, F_{i}$ is of order $\ell-1$ inside $J$, and by definition of $G,|J| \geqslant c_{\ell-1} \geqslant \eta c_{\ell-1}$, the induction hypothesis gives

$$
\frac{|F \cap J|}{|J|} \geqslant(1-\eta)^{2 \ell-2}
$$

Summing over all pieces of $G$ we get, using also (4.6) and (4.4)

$$
|F \cap I| \geqslant|F \cap G| \geqslant(1-\eta)^{2 \ell-2}|G| \geqslant(1-\eta)^{2 \ell}|I|,
$$

which is the first point at order $\ell$.

Moreover, if $r_{J}$ denotes the number of points of $F \cap J$ lying in pieces of $F \cap J$ of size smaller than $c$, then

$$
\frac{r_{J}}{|F \cap J|} \leqslant K_{1}\left(\frac{c}{c_{1}}+\frac{c_{1}}{c_{2}} \frac{1}{(1-\eta)^{4}}+\cdots+\frac{c_{\ell-2}}{c_{\ell-1}} \frac{1}{(1-\eta)^{2 \ell-2}}\right) .
$$

Now let us denote by $r$ the number of points of $F \cap I$ lying in pieces of $F \cap I$ of size smaller than $c$. The contribution to $r$ of points in $G$ is $\sum_{J} r_{J}$ (where the sum ranges over all pieces $J$ of $G$ ), and by the previous inequality, it satisfies

$$
\begin{aligned}
\sum_{J} r_{J} & \leqslant K_{1}\left(\frac{c}{c_{1}}+\frac{c_{1}}{c_{2}} \frac{1}{(1-\eta)^{4}}+\cdots+\frac{c_{\ell-2}}{c_{\ell-1}} \frac{1}{(1-\eta)^{2 \ell-2}}\right)|F \cap G| \\
& \leqslant K_{1}\left(\frac{c}{c_{1}}+\frac{c_{1}}{c_{2}} \frac{1}{(1-\eta)^{4}}+\cdots+\frac{c_{\ell-2}}{c_{\ell-1}} \frac{1}{(1-\eta)^{2 \ell-2}}\right)|F \cap I| .
\end{aligned}
$$

The contribution to $r$ of points in $F \backslash G$ is clearly at most $|F \backslash G|$, which can be bounded above as follows

$$
\begin{aligned}
|(F \cap I) \backslash G| & \leqslant\left|\left(F^{\prime} \cap I\right) \backslash G\right| & & \text { (because } \left.F \subset F^{\prime}\right) \\
& =r^{\prime} & & \text { (by definition of } \left.G \text { and } r^{\prime}\right) \\
& \leqslant K_{1} \frac{c_{\ell-1}}{c_{\ell}}\left|F^{\prime} \cap I\right| & & \text { (by (4.5)) } \\
& \leqslant K_{1} \frac{c_{\ell-1}}{c_{\ell}}|I| & & \\
& \leqslant K_{1} \frac{c_{\ell-1}}{c_{\ell}} \frac{|F \cap I|}{(1-\eta)^{2 \ell}} & & \text { (by (4.7)) }
\end{aligned}
$$

Summing the two contributions and using the above inequalities, we get

$$
r \leqslant K_{1}\left(\frac{c}{c_{1}}+\frac{c_{1}}{c_{2}} \frac{1}{(1-\eta)^{4}}+\cdots+\frac{c_{\ell-2}}{c_{\ell-1}} \frac{1}{(1-\eta)^{2 \ell-2}}+\frac{c_{\ell-1}}{c_{\ell}} \frac{1}{(1-\eta)^{2 \ell}}\right)|F \cap I|,
$$

which is the second point at order $\ell$.

\subsection{Application to the structure of $n$-crossings}

We want now to apply the preceding lemma in order to obtain some statistical results on long range of successive $n$-crossings. We fix some integer $\underline{\ell}$, large enough to satisfy some conditions to be specified later, and we set $\underline{k}:=k(\underline{\ell})$ We define the 
sequences $\left(c_{\ell}\right)_{\ell \geqslant 1}$ and $\left(s_{\ell}\right)_{\ell \geqslant 1}$ as follows.

- $c_{1}:=h_{n_{\ell}}$,

- $s_{1}:=h_{\left(n_{\ell}-\underline{k}\right)}+1$,

- in general, $c_{\ell}:=h_{n_{(\underline{\ell}+\ell-1)}}$, and $s_{\ell}:=h_{n_{(\underline{\ell}+\ell-1)}-k(\underline{\ell}+\ell-1)}+1$.

Using the fact that we always have $h_{n} / h_{n+1}<1 / 3$, we observe that for each $\ell \geqslant 1$, with $k:=k(\underline{\ell}+\ell-1)$,

$$
\frac{s_{\ell}}{c_{\ell}}=\frac{h_{n_{(\underline{\ell}+\ell-1)}-k}+1}{h_{n_{(\underline{\ell}+\ell-1)}}}<2 \frac{h_{n_{(\underline{\ell}+\ell-1)}-k}}{h_{n_{(\underline{\ell}+\ell-1)}}}<\frac{2}{3^{k}} \leqslant \frac{2}{3^{\underline{k}}} .
$$

Hence (4.1) is satisfied if $\underline{\ell}$ is large enough. The fact that (4.2) holds if $\underline{\ell}$ is large enough follows from the following easy consequence of $(2.2)$ :

$$
\frac{h_{n_{\ell}}}{h_{n_{\ell+1}}}<\frac{h_{n_{(\ell+1)}}-(\ell+1)}{h_{n_{(\ell+1)}}}<\frac{1}{3^{\ell+1}} \underset{\ell \rightarrow \infty}{\longrightarrow} 0 .
$$

We can therefore assume that $\underline{\ell}$ is large enough so that both (4.1) and (4.2) hold.

We want to apply Lemma 4.2 to the subsets $F_{i}(i=1, \ldots, d)$ defined by

$$
F_{i}:=\left\{j \in \mathbb{Z}: T^{j} x_{i} \in C_{n_{\underline{\ell}}}\right\} .
$$

Let $I \subset \mathbb{Z}$ be an interval, $n \geqslant 1$ and $i \in\{1, \ldots, d\}$. We say that $x_{i}$ climbs into tower $n$ along $I$ if for each $j \in I, T^{j} x_{i} \in C_{n}$, and there is no $j \in I$ such that $j+1 \in I$, $T^{j} x_{i} \in L_{n}^{h_{n}-1}$ and $T^{j+1} x_{i} \in L_{n}^{0}$. Note that $I$ is included in an $n$-crossing if and only if each coordinate $x_{i}$ climbs into tower $n$ along $I$.

Lemma 4.3. - For each interval $I \subset \mathbb{Z}$ and each $i \in\{1, \ldots, d\}$, if $x_{i}$ climbs into tower $n_{(\underline{\ell}+\ell)}$ along $I$, then $F_{i}$ is of order $\ell$ inside $I$.

Proof. - By construction of the Nearly Finite Chacon Transformation, two successive occurrences of tower $n_{\underline{\ell}}$ inside tower $n_{(\underline{\ell}+1)}$ are separated either by $h_{n_{\ell}-\underline{k}}$ or by $h_{n_{\ell}-\underline{k}}+1$ spacers. Hence, if $x_{i}$ climbs into tower $n_{(\underline{\ell}+1)}$ along $I, F_{i}$ is of order 1 inside $I$. This proves the lemma in the case $\ell=1$.

Assume that the statement of the lemma is true up to $\ell-1$ for some $\ell \geqslant 2$. We consider

We clearly have $F_{i} \subset F_{i}^{\prime}$.

$$
F_{i}^{\prime}:=\left\{j \in \mathbb{Z}: T^{j} x_{i} \in C_{n_{(\underline{\ell}+\ell-1)}}\right\} .
$$

Two successive occurrences of tower $n_{(\underline{\ell}+\ell-1)}$ inside tower $n_{(\underline{\ell}+\ell)}$ are separated either by $h_{n_{(\ell+\ell-1)}-k}$ or by $h_{n_{(\ell+\ell-1)}-k}+1$ spacers, where $k$ is determined by $\ell_{k} \leqslant \underline{\ell}+\ell-1<$ $\ell_{k+1}$. Hence, if $x_{i}$ climbs into tower $n_{(\underline{\ell}+\ell)}$ along $I$, each hole of $F_{i}^{\prime} \cap I$ is of size $\leqslant h_{n_{(\underline{\ell}+\ell-1)}-k}+1=s_{\ell}$, and two consecutive holes of $F_{i}^{\prime} \cap I$ are separated by a piece of $F_{i}^{\prime} \cap I$ of size $h_{n_{(\underline{\ell}+\ell-1)}}=c_{\ell}$. Moreover, along each piece of $F_{i}^{\prime} \cap I, x_{i}$ climbs into tower $n_{(\underline{\ell}+\ell-1)}$. Therefore the property for $\ell-1$ ensures that $F_{i}$ is of order $\ell-1$ inside each piece of $F_{i}^{\prime} \cap I$. It follows that $F_{i}$ is of order $\ell$ inside $\mathrm{I}$, and the lemma is proved by induction.

Proof of Proposition 4.1. - With the subsets $F_{i}$ defined as above, we see that

$$
F:=\bigcap_{1 \leqslant i \leqslant d} F_{i}=\left\{j \in \mathbb{Z}:\left(T^{\times d}\right)^{j} x \in C_{n_{\underline{\ell}}}^{d}\right\} .
$$


Observe that the pieces of $F$ are precisely the $n_{\ell}$-crossings.

Assume that the interval $I \subset \mathbb{Z}$ is included in an $n_{(\ell+\ell)}$-crossing for some $\ell \geqslant 1$ (remember that this is equivalent to: each coordinate $x_{i}$ climbs into tower $n_{(\underline{\ell}+\ell)}$ along $I$ ). Then, putting together Lemma 4.2 and Lemma 4.3, and provided that the length of $I$ be at least $\eta c_{\ell}=\eta h_{n_{(\underline{\ell}+\ell-1)}}$, we get:

- the proportion of $j \in I$ such that $\left(T^{\times d}\right)^{j} x \in C_{n_{\ell}}^{d}$ is at least $(1-\eta)^{2 \ell}$,

- for each $1 \leqslant c \leqslant h_{n_{\ell}}$, the proportion of $j \in F \cap \bar{I}$ belonging to an $n_{\underline{\underline{\ell}}}$-crossing of size $\leqslant c$ is bounded above by

$$
K_{1}\left(\frac{c}{h_{n_{\underline{\ell}}}}+\frac{h_{n_{\underline{\ell}}}}{h_{n_{(\underline{\ell}+1)}}} \frac{1}{(1-\eta)^{4}}+\cdots+\frac{h_{n_{(\underline{\ell}+\ell-2)}}}{h_{n_{(\underline{\ell}+\ell-1)}}} \frac{1}{(1-\eta)^{2 \ell}}\right) .
$$

Let us estimate the general term of the above sum, using the inequality $h_{n_{\ell}} / h_{n_{(\ell+1)}}<$ $1 / 3^{\ell+1}$, and the assumption $(1-\eta)^{2}>1 / 2$.

$$
\begin{aligned}
\frac{h_{n_{(\underline{\ell}+\ell-2)}}}{h_{n_{(\underline{\ell}+\ell-1)}}} \frac{1}{(1-\eta)^{2 \ell}} & <\frac{1}{3(\underline{\ell}+\ell-1)} \frac{1}{(1-\eta)^{2 \ell}} \\
& =\frac{1}{3(\underline{\ell}-1)} \frac{1}{\left(3(1-\eta)^{2}\right)^{\ell}} \\
& <\frac{1}{3(\underline{\ell}-1)}\left(\frac{2}{3}\right)^{\ell} .
\end{aligned}
$$

It follows that there exist a constant $K_{2}$ such that $(4.8) \leqslant K_{1} \frac{c}{h_{n_{\ell}}}+\frac{K_{2}}{3 \underline{\ell}}$.

\subsection{Measure of the edge of $C_{n}^{d}$}

For each $n \geqslant 0$, we say that an $n$-box $L_{n}^{j_{1}} \times \cdots \times L_{n}^{j_{d}}$ is on the edge of $C_{n}^{d}$ if there exists $i \in\{1, \ldots, d\}$ such that $j_{i}=0$ or $j_{i}=h_{n}-1$. We denote by $\partial C_{n}^{d}$ the union of all such $n$-boxes.

As a first application of Proposition 4.1, we have the following result.

Corollary 4.4. -

$$
\delta(n):=\frac{\sigma\left(\partial C_{n}^{d}\right)}{\sigma\left(C_{n}^{d}\right)} \underset{n \rightarrow \infty}{\longrightarrow} 0 .
$$

sketch of proof. - This is a direct consequence of the following facts:

- Since $x$ is typical for $\sigma$, the quotient $\delta(n)$ can be estimated by the ratio

$$
\frac{\sum_{j \in I} \mathbb{1}_{\partial C_{n}^{d}}\left(T^{\times d}\right)^{j} x}{\sum_{j \in I} \mathbb{1}_{C_{n}^{d}}\left(T^{\times d}\right)^{j} x}
$$

for a large interval $I$ containing 0 .

- The subset of $j \in \mathbb{Z}$ such that $\left(T^{\times d}\right)^{j} x \in C_{n}^{d}$ is partitioned into $n$-crossings, and in each $n$-crossing $J$ there are exactly two integers $j$ (the minimum and the maximum of $J$ ) such that $\left(T^{\times d}\right)^{j} x \in \partial C_{n}^{d}$.

- By Proposition 4.1, most $n$-crossings are large if $n$ is large. 


\section{Convergence of sequences of empirical measures}

For each finite subset $J \subset \mathbb{Z}$, we denote by $\gamma_{J}$ the empirical measure

$$
\gamma_{J}:=\sum_{j \in I} \delta_{\left(T^{\times d}\right)^{j} x}
$$

The validity of Property (3.3) whenever $B$ is an $n$-box and $C$ is $C_{n}^{d}$ (remember that $x$ has been chosen as a typical point) means that, if $\left(J_{n}\right)$ is a sequence of intervals containing 0, with $\left|J_{n}\right| \underset{n \rightarrow \infty}{\longrightarrow} \infty$, then we have the convergence $\gamma_{J_{n}} \underset{n \rightarrow \infty}{\longrightarrow} \sigma$.

Our purpose in this section is to extend this convergence to the case where the intervals $J_{n}$ do not necessarily contain 0 , but are not too far from 0 . We will also treat the case where the subsets $J_{n}$ are no longer intervals, but union of intervals with a sufficiently regular structure.

We fix a real number $\varepsilon>0$, small enough so that $(1-\varepsilon)^{2}>1-\eta$. Then we consider an integer $c \geqslant 1$, large enough so that $\frac{c-1}{c}>1-\varepsilon$.

In Sections 5.1 and 5.2, we consider a fixed integer $\underline{\ell}$, large enough so that the result of Proposition 4.1 holds. We can also assume that

$$
K_{1} \frac{c}{h_{n_{\underline{\underline{\ell}}}}}+\frac{K_{2}}{3 \underline{\ell}}<\varepsilon .
$$

We are going to estimate the behaviour of empirical measures with respect to $n_{\underline{\ell}^{-}}$ boxes. The following lemmas are devoted to the control of

$$
\gamma_{I}\left(C_{n_{\underline{\ell}}}^{d}\right)=\sum_{j \in I} \mathbb{1}_{C_{n_{\underline{\ell}}^{d}}}\left(\left(T^{\times d}\right)^{j}(x)\right)
$$

for particular intervals $I$.

\subsection{Consecutive $n$-intervals}

For $n \geqslant 1$, we call $n$-interval any interval $I=\left\{j, j+1, \ldots, j+h_{n}-1\right\}$ of length $h_{n}$ and such that $j$ is a multiple of $h_{n}$. (The second condition is completely artificial, it is only useful to define canonically a cutting of any interval into intervals of length $h_{n}$.)

Lemma 5.1. - Let $p_{1}$ be the smallest integer such that $3^{p_{1}}>2 d+1$ and $p_{1}>d$. There exists a constant $0<\theta_{1}<1$ (depending only on $\eta$ and $d$ ) for which the following holds.

Let $\ell>\underline{\ell}+1$, and let $n$ be such that $n_{(\ell-1)}-k(\ell-1)+p_{1} \leqslant n<n_{\ell}$. Whenever $I_{1}$

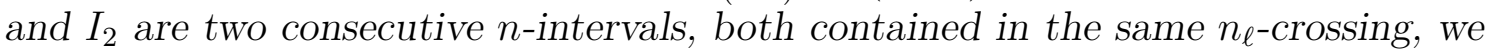
have

$$
\theta_{1} \gamma_{I_{1}}\left(C_{n_{\underline{\ell}}}^{d}\right)<\gamma_{I_{2}}\left(C_{n_{\underline{\underline{\ell}}}}^{d}\right)<\frac{1}{\theta_{1}} \gamma_{I_{1}}\left(C_{n_{\underline{\underline{\ell}}}}^{d}\right)
$$


Proof. - We divide the proof into two cases.

Case 1: $n_{(\ell-1)}+1 \leqslant n \leqslant n_{\ell}$. Set $j_{1}:=\min I_{1}$ and $j_{2}:=\min I_{2}=j_{1}+h_{n}$. Proposition 4.1 applies to $I_{1}$, and this ensures that, among the $\gamma_{I_{1}}\left(C_{n_{\underline{\ell}}}^{d}\right)$ integers $j$ such that $\left(T^{\times d}\right)^{j_{1}+j} x \in C_{n_{\underline{\underline{\ell}}}}^{d}$, a proportion at least $(1-\varepsilon)$ (by $(5.1)$ ) belong to an

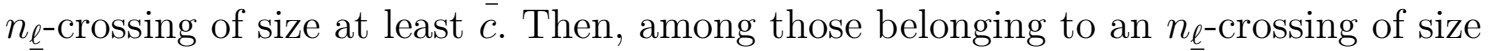
at least $c$, a proportion at least $\frac{c-1}{c}$ are not the minimum of their $n_{\underline{\ell}}$-crossing. By the choice of $\varepsilon$ and $c$, we get the partial following result: a proportion at least $1-\eta$ of integers $j \in\left\{0, \ldots, h_{n}-1\right\}$ are such that, for each $i=1, \ldots, d, T^{j_{1}+j} x_{i} \in C_{n_{\ell}}$, but $T^{j_{1}+j} x_{i}$ is not in the bottom level of tower $n_{\underline{\ell}}$. Let us consider such an integer $j$.

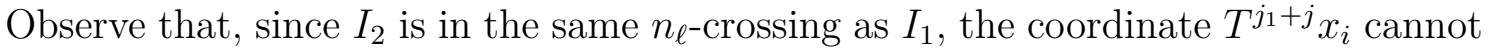
be in the last occurrence of tower $n$ inside tower $n_{\ell}$. Hence it will pass through zero or one spacer before coming back to $C_{n}$. Then we can use a similar argument as in the proof of Lemma 3.8: according to whether coordinate $i$ sees a spacer or not, $T^{j_{2}+j} x_{i}=T^{j_{1}+j+h_{n}} x_{i}$ is either in the same level of tower $n$ as $T^{j_{1}+j} x_{i}$, or in the level immediately below. And the same applies if we consider the levels of tower $n_{\underline{\ell}}$. Hence $T^{j_{2}+j} x_{i} \in C_{n_{\underline{\ell}}}$.

This proves that $\gamma_{I_{2}}\left(C_{n_{\ell}}^{d}\right) \geqslant(1-\eta) \gamma_{I_{1}}\left(C_{n_{\ell}}^{d}\right)$. But we can do a similar reasoning starting from $I_{2}$ and going backwards, and we get the announced inequalities for any $0<\theta_{1} \leqslant(1-\eta)$.

Case 2: $n_{(\ell-1)}-k(\ell-1)+p_{1} \leqslant n \leqslant n_{(\ell-1)}$. - To simplify the notations, we set $n^{\prime}:=n_{(\ell-1)}-k(\ell-1)$. The reason why we cannot do the same reasoning as in the previous case is the following: when for some $j$ the coordinate $T^{j} x_{i}$ leaves tower $n$, it will come back to $C_{n}$ after $0,1, h_{n^{\prime}}$ or $h_{n^{\prime}}+1$ spacers. Because of this huge number of spacers that might separate two climbings into tower $n$, we cannot be sure that $T^{j+h_{n}} x_{i}$ will be in $C_{n_{\ell}}$. To circumvent this difficulty, we introduce what we call the fake tower $n^{\prime}$ : it is the Rokhlin tower of height $h_{n^{\prime}}$ whose levels are the $h_{n^{\prime}}$ spacers placed on top of tower $n_{(\ell-1)}$ in the construction. Let us denote by $\tilde{L}_{n^{\prime}}^{0}, \ldots, \tilde{L}_{n^{\prime}}^{h_{n^{\prime}}-1}$ its consecutive levels. We note that this fake tower $n^{\prime}$ is disjoint from $C_{n_{(\ell-1)}}$, a fortiori it is disjoint from $C_{n_{\underline{\ell}}}$. However we can construct a fake $C_{n_{\underline{\ell}}}$ inside the fake tower $n^{\prime}$ by mimicking the structure of $C_{n_{\underline{\ell}}}$ inside tower $n^{\prime}$. More precisely, we set

$$
\tilde{C}_{n_{\underline{\ell}}}:=\bigsqcup_{j: L_{n^{\prime}}^{j} \subset C_{n_{\underline{\ell}}}} \tilde{L}_{n^{\prime}}^{j}, \quad \text { and } \quad \bar{C}_{n_{\underline{\ell}}}:=C_{n_{\underline{\ell}}} \sqcup \tilde{C}_{n_{\underline{\ell}}} .
$$

If we consider $\bar{C}_{n_{\ell}}$ instead of $C_{n_{\ell}}$, then everything happens as if the $n$-intervals were both contained in a single $n_{(\ell-1)}$-crossing. Hence we can use Case 1 with $(\ell-1)$ in place of $\ell$, which yields

$$
(1-\eta) \gamma_{I_{1}}\left(\bar{C}_{n_{\underline{\ell}}}^{d}\right)<\gamma_{I_{2}}\left(\bar{C}_{n_{\underline{\ell}}}^{d}\right)<\frac{1}{(1-\eta)} \gamma_{I_{1}}\left(\bar{C}_{n_{\underline{\ell}}}^{d}\right) .
$$

It remains now to compare $\gamma_{I}\left(\bar{C}_{n_{\underline{\underline{\ell}}}}^{d}\right)$ with $\gamma_{I}\left(C_{n_{\underline{\ell}}}^{d}\right)$ for $I=I_{1}$ or $I=I_{2}$.

For this we will consider $n^{\prime}$-intervals intersecting $I$. Let $J$ be such an $n^{\prime}$-interval. We say that it is suspect if there exists $1 \leqslant i \leqslant d$ and $j \in J$ such that $T^{j} x_{i} \notin C_{n_{(\ell-1)}}$. Note that, by definition of a suspect interval, if $J \subset I$ is an $n^{\prime}$-interval which is not 
suspect, then $\gamma_{J}\left(C_{n_{\underline{\ell}}}^{d}\right)=\gamma_{J}\left(\bar{C}_{n_{\underline{\ell}}}^{d}\right)$. Observe also that, when $T^{j} x_{i}$ leaves $C_{n_{(\ell-1)}}$, then it comes back after at most $h_{n^{\prime}}+1$ spacers (remember that everything takes place inside an $n_{\ell^{-}}$crossing, therefore the coordinates do not leave $C_{n_{\ell}}$ ). Moreover, when it comes back to $C_{n_{(\ell-1)}}$, it stays in $C_{n_{(\ell-1)}}$ for a time $\geqslant h_{n_{(\ell-1)}} \geqslant h_{n}$. Since $|I|=h_{n}$, each coordinate $1 \leqslant i \leqslant d$ is responsible for at most 2 suspect $n^{\prime}$-intervals intersecting $I$, and we conclude that there exist at most $2 d$ suspect $n^{\prime}$-intervals intersecting $I$. Moreover, since we assumed that $n \geqslant n^{\prime}+p_{1}$, we have $h_{n} / h_{n^{\prime}}>3^{p_{1}}>2 d+1$, and this ensures that there exists at least one $n^{\prime}$-interval contained in $I$ which is not suspect.

Now if $J^{\prime}$ is a suspect interval intersecting $I$, we can find a chain $J^{\prime}=J_{0}^{\prime}, J_{1}^{\prime}, \ldots, J_{r}^{\prime}=$ $J$ of consecutive $n^{\prime}$-intervals, where $J_{0}^{\prime}, \ldots, J_{r-1}^{\prime}$ are suspect (hence $r \leqslant 2 d$ ), $J=J_{r}^{\prime}$ is not suspect and contained in $I$. Applying Case $1 r \leqslant 2 d$ times (with $n^{\prime}$ in place of $n$ and $\ell-1$ in place of $\ell$ gives

$$
\gamma_{I}\left(C_{n_{\underline{\ell}}}^{d}\right) \geqslant \gamma_{J}\left(C_{n_{\underline{\ell}}}^{d}\right)=\gamma_{J}\left(\bar{C}_{n_{\underline{\ell}}}^{d}\right) \geqslant(1-\eta)^{2 d} \gamma_{J^{\prime}}\left(\bar{C}_{n_{\underline{\ell}}}^{d}\right) \geqslant(1-\eta)^{2 d} \gamma_{J^{\prime}}\left(\tilde{C}_{n_{\underline{\ell}}}^{d}\right) .
$$

Since only suspect intervals can contribute to $\gamma_{I}\left(\tilde{C}_{n_{\underline{\ell}}}^{d}\right)$, and since there are at most $2 d$ of them, summing the preceding inequality over all suspect intervals $J^{\prime}$ intersecting $I$ yields

$$
2 d \gamma_{I}\left(C_{n_{\underline{\underline{\ell}}}}^{d}\right) \geqslant(1-\eta)^{2 d} \gamma_{I}\left(\tilde{C}_{n_{\underline{\ell}}}^{d}\right)
$$

In other words,

$$
\gamma_{I}\left(\tilde{C}_{n_{\underline{\ell}}}^{d}\right) \leqslant \frac{2 d}{(1-\eta)^{2 d}} \gamma_{I}\left(C_{n_{\underline{\ell}}}^{d}\right)
$$

Adding $\gamma_{I}\left(C_{n_{\underline{\ell}}}^{d}\right)$ on both sides, we get

$$
\gamma_{I}\left(\bar{C}_{n_{\underline{\underline{\ell}}}}^{d}\right) \leqslant\left(\frac{2 d}{(1-\eta)^{2 d}}+1\right) \gamma_{I}\left(C_{n_{\underline{\underline{\ell}}}}^{d}\right) .
$$

Inserting the above inequality for $I=I_{1}$ in (5.2), we get

$$
\gamma_{I_{2}}\left(C_{n_{\underline{\ell}}}^{d}\right) \leqslant \gamma_{I_{2}}\left(\bar{C}_{n_{\underline{\underline{\ell}}}}^{d}\right)<\frac{1}{\theta_{1}} \gamma_{I_{1}}\left(C_{n_{\underline{\underline{\ell}}}}^{d}\right)
$$

with

$$
\theta_{1}:=(1-\eta)\left(\frac{2 d}{(1-\eta)^{2 d}}+1\right)^{-1} .
$$

But we can exchange the roles of $I_{1}$ and $I_{2}$ and this gives the announced result.

Remark 5.2. - Let $\ell$ and $n$ be as in Lemma 5.1. Assume that $I_{1}$ and $I_{2}$ are consecutive $n$-intervals, but only $I_{1}$ is supposed to be contained in some $n_{\ell}$ crossing $J$. Then we get the inequality

$$
\gamma_{I_{2} \cap J}\left(C_{n_{\underline{\underline{\ell}}}}^{d}\right)<\frac{1}{\theta_{1}} \gamma_{I_{1}}\left(C_{n_{\underline{\ell}}}^{d}\right) .
$$

Indeed, we can always change what happens on $I_{2} \backslash J$ to do as if both $I_{1}$ and $I_{2}$ were included in the same $n_{\ell}$-crossing. 


\subsection{Contribution of substantial subintervals}

Lemma 5.3. - Let $p_{2}$ be the smallest integer such that $\frac{1}{3^{p_{2}}}<\frac{1}{3} \eta$. For each $M>0$, there exists a real number $0<\theta_{2}(M)<1$ (depending also on $\eta$ and $d$ ) for which the following holds.

Let $\ell>\underline{\ell}+1$, and let $n$ be such that

$$
n_{(\ell-1)}-k(\ell-1)+p_{1}+p_{2} \leqslant n<n_{\ell} .
$$

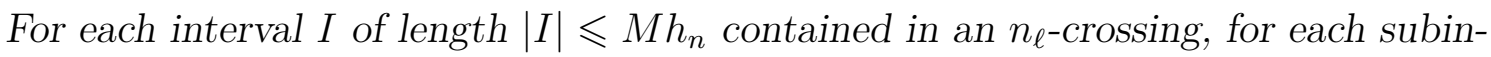
terval $J \subset I$ with $|J| \geqslant \eta h_{n}$, we have

$$
\gamma_{J}\left(C_{n_{\underline{\underline{\ell}}}}^{d}\right) \geqslant \theta_{2}(M) \gamma_{I}\left(C_{n_{\underline{\underline{\ell}}}}^{d}\right) \text {. }
$$

Remark 5.4. - Note that if $\ell$ is large enough, we have $n_{(\ell-1)}-k(\ell-1)+p_{1}+p_{2}<$ $n_{(\ell-1)}$, hence the above is valid in particular for $n_{(\ell-1)} \leqslant n<n_{\ell}$.

Proof. - Under the assumptions of the lemma, we have

$$
n_{\underline{\ell}}<n_{(\ell-1)}-k(\ell-1)+p_{1} \leqslant n-p_{2}<n_{\ell} .
$$

We consider the $\left(n-p_{2}\right)$-intervals included in $I$, and we will apply Lemma 5.1 to them. Remember that $h_{n-p_{2}} \geqslant \frac{1}{7^{p_{2}}} h_{n}$. Hence the number of $\left(n-p_{2}\right)$-intervals contained in $I$ is at most $7^{p_{2}} M$. Moreover their length $h_{n-p_{2}}$ satisfies $h_{n-p_{2}}<\frac{1}{3^{p_{2}}} h_{n}<\frac{1}{3}|J|$. Hence there is at least one $\left(n-p_{2}\right)$-interval included in $J$. Let us call it $J^{\prime}$. Now, if $I^{\prime}$ is another $\left(n-p_{2}\right)$-interval contained in $I$, a repeated use of Lemma 5.1 yields

$$
\gamma_{J}\left(C_{n_{\underline{\ell}}}^{d}\right) \geqslant \gamma_{J^{\prime}}\left(C_{n_{\underline{\ell}}}^{d}\right) \geqslant \theta_{1}^{7^{p_{2} M}} \gamma_{I^{\prime}}\left(C_{n_{\underline{\ell}}}^{d}\right) .
$$

There might also exist two $\left(n-p_{2}\right)$ intervals intersecting $I$ at its extremities but not contained in $I$, hence not necessarily contained in the $n_{\ell^{-}}$-crossing. If $I^{\prime}$ is such an interval, we use Remark 5.2 and get the same inequality (with $\gamma_{I^{\prime} \cap I}$ instead of $\gamma_{I^{\prime}}$ ). Summing over all the $\left(n-p_{2}\right)$-intervals intersecting $I$, we get

$$
\left(7^{p_{2}} M+2\right) \gamma_{J}\left(C_{n_{\underline{\ell}}}^{d}\right) \geqslant \theta_{1}^{7^{p_{2} M}} \gamma_{I}\left(C_{n_{\underline{\ell}}}^{d}\right) \text {. }
$$

This gives the announced result, with $\theta_{2}(M):=\theta_{1}^{7^{p_{2}} M} /\left(7^{p_{2}} M+2\right)$.

\subsection{How to apply Proposition 3.5}

Here we want to provide some conditions so that Proposition 3.5 applies to a sequence of empirical measures $\left(\gamma_{J_{m}}\right)$ for some sequence of intervals $\left(J_{m}\right)$. We make the following assumptions.

(5.4) For each $m$, there exists an integer $\ell_{m}$ with $\ell_{m} \rightarrow \infty$ as $m \rightarrow \infty$, such that $J_{m}$ is contained in some $n_{\ell_{m}}$-crossing,

and

(5.5) For each $m$, there exists an integer $n(m)$ satisfying

- $n_{\left(\ell_{m}-1\right)}-k\left(\ell_{m}-1\right)+p_{1}+2 p_{2} \leqslant n(m)<n_{\ell_{m}}$,

- $\eta h_{n(m)} \leqslant\left|J_{m}\right| \leqslant h_{n(m)}$, 
Note that, as soon as $\underline{\ell}$ is large enough so that Proposition 4.1 applies, the first point of this proposition ensures that $\gamma_{J_{m}}\left(C_{n_{\ell}}^{d}\right)>0$ for $m$ large enough, which is the first assumption needed to apply Proposition 3.5.

It remains, for some fixed $\underline{\ell}$ and $\ell$, to control the ratio $\gamma_{J_{m}}\left(C_{n_{(\underline{\ell}+\ell)}}^{d}\right) / \gamma_{J_{m}}\left(C_{n_{\underline{\ell}}}^{d}\right)$, which is the purpose of the following lemma.

Lemma 5.5. - Let $\underline{\ell}$ be large enough so that Proposition 4.1 applies and (5.1) holds. Assume also that

$$
\left(K_{1}+K_{2}\right) \frac{1}{3 \underline{\ell}}<\eta
$$

Let $\ell \geqslant 1$, and let $\left(J_{m}\right)$ be a sequence of intervals satisfying (5.4) and (5.5). Then for each $m$ large enough

$$
\gamma_{J_{m}}\left(C_{n_{\underline{\ell}}}^{d}\right) \geqslant(1-\eta)^{2 \ell+1} \theta_{2}(7) \gamma_{J_{m}}\left(C_{n_{(\underline{\ell}+\ell)}}^{d}\right) .
$$

Proof. - We first consider the case where $n(m) \geqslant n_{\left(\ell_{m}-1\right)}$. Then we can apply Proposition 4.1 (with $\underline{\ell}+\ell$ in place of $\underline{\ell}$ ) to show that, if $\ell_{m} \geqslant \underline{\ell}+\ell+1$, the proportion of integers in $\left\{j \in J_{m}:\left(T^{\times d}\right)^{j} x \in C_{n_{(\underline{\ell}+\ell)}}^{d}\right\}$ belonging to an $n_{(\underline{\ell}+\ell)}$-crossing of size less than $h_{n_{(\underline{\ell}+\ell-1)}}$ is bounded above by

$$
K_{1} \frac{h_{n_{(\underline{\ell}+\ell-1)}}}{h_{n_{(\underline{\ell}+\ell)}}}+\frac{K_{2}}{3 \underline{\ell}+\ell} \leqslant\left(K_{1}+K_{2}\right) \frac{1}{3 \underline{\ell}+\ell}<\eta .
$$

Now, if $I \subset J_{m}$ is an $n_{(\underline{\ell}+\ell)}$-crossing with $|I| \geqslant h_{n_{(\underline{\ell}+\ell-1)}}$, another application of Proposition 4.1 proves that the proportion of integers $j \in I$ such that $\left(T^{\times d}\right)^{j} x \in C_{n_{\underline{\ell}}}^{d}$ is at least $(1-\eta)^{2 \ell}$. We finally get in this case

$$
\gamma_{J_{m}}\left(C_{n_{\underline{\ell}}}^{d}\right) \geqslant(1-\eta)^{2 \ell+1} \gamma_{J_{m}}\left(C_{n_{(\underline{\ell}+\ell)}^{d}}^{d}\right) .
$$

Now we consider the case where $n_{\left(\ell_{m}-1\right)}-k\left(\ell_{m}-1\right)+p_{1}+2 p_{2} \leqslant n(m)<n_{\left(\ell_{m}-1\right)}$. Let $n$ be the largest integer, $n \leqslant n(m)$, such that $h_{n} \leqslant\left|J_{m}\right|$. If $n<n(m)$, then we have $h_{n+1}>\left|J_{m}\right| \geqslant \eta h_{n(m)}$. But on the other hand, $h_{n+1}<h_{n(m)} / 3^{n(m)-n-1}$. Taking into account the definition of $p_{2}$ (Lemma 5.3), we get that $n(m)-n<p_{2}$, and finally that $n$ satisfies

$$
n_{\left(\ell_{m}-1\right)}-k\left(\ell_{m}-1\right)+p_{1}+p_{2}<n<n_{\left(\ell_{m}-1\right)} .
$$

Since $\left|J_{m}\right|<h_{n_{\left(\ell_{m}-1\right)}}$, we observe that each coordinate can leave $C_{n_{\left(\ell_{m}-1\right)}}$ only once on $J_{m}$, and when it does so, it stays outside $C_{n_{\left(\ell_{m}-1\right)}}$ on an interval of length $\leqslant h_{n_{\left(\ell_{m}-1\right)}-k\left(\ell_{m}-1\right)}+1$. Since there are $d$ coordinates, the set of integers $j \in J_{m}$ such that $\left(T^{\times d}\right)^{j} x \in C_{n_{\left(\ell_{m}-1\right)}}^{d}$ is cut into at most $(d+1)$ pieces, and its cardinality is at 
least

$$
\begin{aligned}
\left|J_{m}\right|-d\left(h_{n_{\left(\ell_{m}-1\right)}-k\left(\ell_{m}-1\right)}+1\right) & \geqslant\left|J_{m}\right|-\frac{d}{3^{p_{1}+p_{2}}} h_{n} \\
& \geqslant\left|J_{m}\right|\left(1-\frac{d}{3^{p_{1}+p+2}}\right) \\
& \geqslant\left|J_{m}\right|\left(1-\frac{\eta}{6}\right) \geqslant \frac{\left|J_{m}\right|}{2}
\end{aligned}
$$

Therefore, there exists at least one subinterval $\tilde{J}_{m} \subset J_{m}$, with size

$$
\left|\tilde{J}_{m}\right| \geqslant \frac{\left|J_{m}\right|}{2(d+1)} \geqslant \eta h_{n}
$$

and which is contained in a single $n_{\left(\ell_{m}-1\right)}$-crossing. Since $n_{\left(\ell_{m}-2\right)}<n<n_{\left(\ell_{m}-1\right)}$, the estimation (5.7) is valid for $\tilde{J}_{m}$ in place of $J_{m}$, i.e.

$$
\gamma_{\tilde{J}_{m}}\left(C_{n_{\underline{\ell}}}^{d}\right) \geqslant(1-\eta)^{2 \ell+1} \gamma_{\tilde{J}_{m}}\left(C_{n_{(\underline{\ell}+\ell)}}^{d}\right) .
$$

Note that, if $n<n(m)$, then by definition of $n$ we have $\left|J_{m}\right|<h_{n+1}<7 h_{n}$. If $n=n(m),\left|J_{m}\right|=h_{n(m)}=h_{n}$. Hence in all cases we have $\eta h_{n} \leqslant\left|\tilde{J}_{m}\right| \leqslant\left|J_{m}\right| \leqslant 7 h_{n}$. So we can also apply Lemma 5.3 with $I:=J_{m}, J:=\tilde{J}_{m}$, and $\underline{\ell}+\ell$ in place of $\underline{\ell}$. This yields

$$
\gamma_{\tilde{J}_{m}}\left(C_{n_{(\underline{\ell}+\ell)}}^{d}\right) \geqslant \theta_{2}(7) \gamma_{J_{m}}\left(C_{n_{(\underline{\ell}+\ell)}}^{d}\right) .
$$

Combining (5.8) and (5.9), we get

$$
\begin{aligned}
\gamma_{J_{m}}\left(C_{n_{\underline{\ell}}}^{d}\right) & \geqslant \gamma_{\tilde{J}_{m}}\left(C_{n_{\underline{\ell}}}^{d}\right) \\
& \geqslant(1-\eta)^{2 \ell+1} \gamma_{\tilde{J}_{m}}\left(C_{n_{(\underline{\ell}+\ell)}^{d}}^{d}\right) \\
& \geqslant(1-\eta)^{2 \ell+1} \theta_{2}(7) \gamma_{J_{m}}\left(C_{n_{(\underline{\ell}+\ell)}^{d}}^{d}\right) .
\end{aligned}
$$

With the above lemma, we see that all the conditions needed to apply Proposition 3.5 are satisfied, and this gives the following result.

Lemma 5.6. - Let $\left(J_{m}\right)$ be a sequence of intervals satisfying (5.4) and (5.5). Then there is a subsequence $\left(\gamma_{J_{m_{j}}}\right)$ which converges to some nonzero Radon measure.

\subsection{Convergence of sequences of empirical measures}

Proposition 5.7. - Let $\left(I_{m}\right)$ and $\left(J_{m}\right)$ be two sequences of intervals, with $J_{m} \subset I_{m}$. Assume that there exist two sequences of integers $\left(\ell_{m}\right)$ and $(n(m))$, and a real number $M>0$ such that

- $\ell_{m} \rightarrow \infty$

- $n_{\left(\ell_{m}-1\right)}-k\left(\ell_{m}-1\right)+p_{1}+2 p_{2} \leqslant n(m)<n_{\ell_{m}}$,

- $I_{m}$ is contained in some $n_{\ell_{m}}$-crossing,

- $\eta h_{n(m)} \leqslant\left|J_{m}\right| \leqslant h_{n(m)}$, 
- $\left|I_{m}\right| \leqslant M h_{n(m)}$.

If $\gamma_{I_{m}} \underset{m \rightarrow \infty}{\longrightarrow} \sigma$, we also have $\gamma_{J_{m}} \underset{m \rightarrow \infty}{\longrightarrow} \sigma$.

Proof. - The assumptions (5.4) and (5.5) are satisfied for the sequence of intervals $\left(J_{m}\right)$, hence Lemma 5.6 applies to the sequence of measures $\left(\gamma_{J_{m}}\right)$. Therefore, it is enough to prove that, if $\gamma_{J_{m}}$ converges to some nonzero Radon measure $\gamma$, then $\gamma=\sigma$ up to some multiplicative constant. So, let us assume that $\gamma_{J_{m}} \rightarrow \gamma$. Since $J_{m} \subset I_{m}$, we have $\gamma_{J_{m}} \leqslant \gamma_{I_{m}}$. We can also apply Lemma 5.3 which shows that, for each large enough integer $\underline{\ell}$, we have as soon as $n(m)>n_{\underline{\ell}}+1$

$$
\gamma_{J_{m}}\left(C_{n_{\underline{\ell}}}^{d}\right) \geqslant \theta_{2}(M) \gamma_{I_{m}}\left(C_{n_{\underline{\ell}}}^{d}\right) \text {. }
$$

Then Proposition 3.6 ensures that $\gamma \ll \sigma$. Now by ergodicity of $\left(X^{d}, \sigma, T^{\times d}\right)$, it only remains to show that $\gamma$ is $T^{\times d}$-invariant. For this we want to apply Lemma 3.2. Since $X_{\mathbf{0}}^{d} \subset X^{d} \backslash X_{\infty}^{d}$, we have $\sigma\left(X_{\mathbf{0}}^{d}\right)=0$ hence $\gamma\left(X_{\mathbf{0}}^{d}\right)=0$ by absolute continuity. Finally, observe that if for some fixed integer $n, B$ and $B^{\prime}$ are two $n$-boxes contained in the same $n$-diagonal, then for any $m$, as $J_{m}$ is an interval,

$$
\left|\gamma_{J_{m}}(B)-\gamma_{J_{m}}\left(B^{\prime}\right)\right| \leqslant 1
$$

Indeed, the times $j$ when the orbit of $x$ falls in $B$ alternate with the times when the orbit of $x$ fall in $B^{\prime}$. On the other hand the first point of Proposition 4.1 ensures that

$$
\gamma_{J_{m}}\left(C_{n}^{d}\right) \underset{m \rightarrow \infty}{\longrightarrow} \infty
$$

and it follows that $\gamma(B)=\gamma\left(B^{\prime}\right)$. Lemma 3.2 now proves that $\gamma$ is $T^{\times d}$-invariant.

Remark 5.8. - Note that the condition $\gamma_{I_{m}} \underset{m \rightarrow \infty}{\longrightarrow} \sigma$ is automatically satisfied if $0 \in I_{m}$ for each $m$, since we took $x$ as a typical point for $\sigma$.

Now we want to extend Proposition 5.7 to the case where $\left(J_{m}\right)$ is no longer a sequence of intervals, but $J_{m}$ is a subset of $I_{m}$ with a sufficiently regular structure.

Proposition 5.9. - Let $\left(I_{m}\right)$ and $\left(J_{m}\right)$ be two sequences of finite subsets of $\mathbb{Z}$, and let $M>0$. We assume that the following conditions are satisfied.

- $J_{m} \subset I_{m}$ for each $m$.

- There exists a sequence of integers $\left(\ell_{m}\right)$ with $\ell_{m} \rightarrow \infty$ as $m \rightarrow \infty$, such that for each $m, I_{m}$ is an interval contained in some $n_{\ell_{m}}$-crossing.

- There exists a sequence of integers $(n(m))$ with

$$
n_{\left(\ell_{m}-1\right)}-k\left(\ell_{m}-1\right)+p_{1}+2 p_{2} \leqslant n(m)<n_{\ell_{m}},
$$

such that for each $m, J_{m}$ is a disjoint union of intervals of common size $s(m)$, where $\eta h_{n(m)} \leqslant s(m) \leqslant h_{n(m)}$, and $I_{m} \backslash J_{m}$ does not contain an interval of size greater than $M h_{n(m)}$.

If $\gamma_{I_{m}} \underset{m \rightarrow \infty}{\longrightarrow} \sigma$, then we also have $\gamma_{J_{m}} \underset{m \rightarrow \infty}{\longrightarrow} \sigma$.

Proof. - We just have to justify that the same arguments as in the proof of Proposition 5.7 apply also in this case. First, we want to prove that the conclusion of Lemma 5.6 holds for $\left(J_{m}\right)$. For this, it is enough to observe that all the pieces of 
$J_{m}$ satisfy assumptions (5.4) and (5.5). Hence the estimation given in Lemma 5.5 is valid for each piece of $J_{m}$, and then it is also valid for $J_{m}$ itself.

Now, let $\underline{\ell}$ be a large enough integer, and take $m$ large enough so that $n(m)>n_{\ell}+1$. Let $J$ be any piece of $J_{m}$ (in particular we have $|J|=s(m) \geqslant \eta h_{n(m)}$ ), and let $\bar{I}$ be the interval constituted of $J$ and the two adjacent pieces of $I_{m} \backslash J_{m}$. Then we have $|I| \leqslant 40 h_{n(m)}+s(m) \leqslant 100 h_{n(m)}$, and we can apply Lemma 5.3 to $I$ and $J$ to get

$$
\gamma_{J}\left(C_{n_{\underline{\underline{\ell}}}}^{d}\right) \geqslant \theta_{2} \gamma_{I}\left(C_{n_{\underline{\underline{\ell}}}}^{d}\right) \text {. }
$$

Summing over all pieces $J$ of $J_{m}$, we get

$$
\gamma_{J_{m}}\left(C_{n_{\underline{\ell}}}^{d}\right) \geqslant \theta_{2} \gamma_{I_{m}}\left(C_{n_{\underline{\ell}}}^{d}\right)
$$

which is the second key step in the proof of Proposition 5.7. This ensures that, if $\gamma_{J_{m}} \rightarrow \gamma$, then $\gamma \ll \sigma$.

Finally, we have to see that $\gamma$ is $T^{\times d}$-invariant, and it is enough for that to show that, if for some fixed $n$ we consider two $n$-boxes $B$ and $B^{\prime}$ on the same $n$-diagonal, then $\gamma(B)=\gamma\left(B^{\prime}\right)$. But for each $m$ and each piece $J$ of $J_{m}$, we have $\left|\gamma_{J}(B)-\gamma_{J}\left(B^{\prime}\right)\right| \leqslant 1$, whereas by the first point of Proposition 4.1, we know that

$$
\min _{J \text { piece of } J_{m}} \gamma_{J}\left(C_{n_{\underline{\ell}}}^{d}\right) \underset{m \rightarrow \infty}{\longrightarrow} \infty
$$

\section{Twisting transformations and decomposition of $\sigma$ as a product}

The purpose of this section is to provide a criterion ensuring that $\sigma$ can be decomposed into the product of two measures $\sigma_{1} \times \sigma_{2}, \sigma_{i}$ being a $T^{\times d_{i}}$-invariant Radon measure on $X^{d_{i}}$ for some $1 \leqslant d_{i}<d, d_{1}+d_{2}=d$. We will need for that to introduce the following type of transformation of $X^{d}$.

Definition 6.1. - The transformation $S: X^{d} \rightarrow X^{d}$ is said to be a twisting transformation if there exists a partition $\{1, \ldots, d\}=G_{0} \sqcup G_{1}$ into two nonempty subsets such that for each $\left(y_{1}, \ldots, y_{d}\right) \in X^{d}$,

$$
S\left(y_{1}, \ldots, y_{d}\right)=\left(z_{1}, \ldots, z_{d}\right), \text { where } z_{i}:= \begin{cases}T y_{i} & \text { if } i \in G_{1} \\ y_{i} & \text { if } i \in G_{0}\end{cases}
$$

The reason why we introduce those twisting transformations is that, if we are able to prove that $\sigma$ is invariant by some twisting transformation then $\sigma$ can be decomposed as a product of two measures. More precisely, by Theorem A.1 in [JRdlR18] we have the following result.

Proposition 6.2. - Assume that $\sigma$ is invariant by some twisting transformation $S$, and let $\{1, \ldots, d\}=G_{0} \sqcup G_{1}$ be the partition associated with $S$. Then there exist Radon measures $\sigma_{0}$ and $\sigma_{1}$ on $X^{G_{0}}$ and $X^{G_{1}}$ respectively, such that

- $\sigma=\sigma_{0} \otimes \sigma_{1}$;

- each $\sigma_{s}$ is $T^{\times\left|G_{a}\right|}$-invariant $(a=0,1)$, and the system $\left(X^{G_{a}}, T^{\times\left|G_{a}\right|}, \sigma_{a}\right)$ is conservative ergodic. 
Thus, if the assumption of the above proposition is satisfied, we can write $\sigma$ as the product of two measures which are invariant by some smaller Cartesian power of $T$, and to which we can apply the induction hypothesis to finish the proof of Theorem 3.10. We want now to give a condition under which we are able to prove that $\sigma$ is indeed invariant by some twisting transformation.

In the next proposition, we use again the notation $\Omega_{n}$ which was introduced in the proof of Lemma 3.2: recall that $\Omega_{n}$ is the union of all $n$-boxes of the form $L_{n}^{j_{1}} \times \cdots \times L_{n}^{j_{d}}$ where for all $i=1, \ldots, d, j_{i} \neq 0$. We observe that, if $B$ is an $n$-box contained in $\Omega_{n}$ and if $S$ is a twisting transformation, then $S^{-1} B$ is also an $n$-box (but not necessarily contained in $\Omega_{n}$ ). Note also that $\Omega_{n} \subset \Omega_{n+1}$ for each $n$.

Proposition 6.3. - Assume that there exist $\left(\sigma_{n}^{\prime}\right),\left(\sigma_{n}\right)$, two sequences of Radon measures on $X^{d}$, and a sequence $\left(S_{n}\right)$ of twisting transformations satisfying

- $\sigma_{n} \underset{n \rightarrow \infty}{\longrightarrow} \sigma$,

- $\sigma_{n}^{\prime} \underset{n \rightarrow \infty}{\longrightarrow} \sigma$,

- For each $m \geqslant 0$, and for $n$ large enough (depending on $m$ ), for each $m$-box $B \subset \Omega_{m}, \sigma_{n}^{\prime}\left(S_{n}^{-1} B\right)=\sigma_{n}(B)$.

Then there exists a twisting transformation $S$ such that $\sigma$ is $S$-invariant. In particular, $\sigma$ is a product measure as in Proposition 6.2.

Proof. - Note that, $d$ being fixed here, there exist only finitely many twisting transformations. Therefore, considering subsequences if necessary, we may assume that there exist a twisting transformation $S$ such that $S_{n}=S$ for each $n$.

Now, let $m \geqslant 0$ be large enough so that $\delta(m)<1 / 2$ (see Corollary 4.4). In particular, $\sigma\left(C_{m}^{d}\right)>0$. Let $m^{\prime} \geqslant m$. Then, for each $n$ large enough (depending on $m^{\prime}$ ), if $B$ is an $m^{\prime}$-box contained in $\Omega_{m}$, then $B \subset \Omega_{m^{\prime}}$ and we know that $\sigma_{n}^{\prime}\left(S^{-1} B\right)=\sigma_{n}(B)$. By Remark 3.4, the assumptions of the lemma also yield

$$
\frac{\sigma_{n}^{\prime}\left(S^{-1} B\right)}{\sigma_{n}^{\prime}\left(C_{m}^{d}\right)} \underset{n \rightarrow \infty}{\longrightarrow} \frac{\sigma\left(S^{-1} B\right)}{\sigma\left(C_{m}^{d}\right)} .
$$

But the left-hand side of the above formula is equal to

where

$$
\frac{\sigma_{n}^{\prime}\left(S^{-1} B\right)}{\sigma_{n}^{\prime}\left(C_{m}^{d}\right)}=\frac{\sigma_{n}(B)}{\sigma_{n}\left(C_{m}^{d}\right)} \frac{\sigma_{n}\left(C_{m}^{d}\right)}{\sigma_{n}^{\prime}\left(C_{m}^{d}\right)}
$$

$$
\frac{\sigma_{n}(B)}{\sigma_{n}\left(C_{m}^{d}\right)} \underset{n \rightarrow \infty}{\longrightarrow} \frac{\sigma(B)}{\sigma\left(C_{m}^{d}\right)}
$$

It remains to control the ratio $\sigma_{n}\left(C_{m}^{d}\right) / \sigma_{n}^{\prime}\left(C_{m}^{d}\right)$. For this, we write

$$
\sigma_{n}^{\prime}\left(C_{m}^{d}\right)=\sigma_{n}^{\prime}\left(S^{-1} \Omega_{m}\right)+\sigma_{n}^{\prime}\left(C_{m}^{d} \backslash S^{-1} \Omega_{m}\right),
$$

and

$$
\sigma_{n}\left(C_{m}^{d}\right)=\sigma_{n}\left(\Omega_{m}\right)+\sigma_{n}\left(C_{m}^{d} \backslash \Omega_{m}\right) .
$$

We observe that the first terms $\sigma_{n}^{\prime}\left(S^{-1} \Omega_{m}\right)$ and $\sigma_{n}\left(\Omega_{m}\right)$ are equal. Moreover, as $C_{m}^{d} \backslash \Omega_{m}$ and $C_{m}^{d} \backslash S^{-1} \Omega_{m}$ are included in $\partial C_{m}^{d}$, we have by Corollary 4.4

$$
\frac{\sigma_{n}\left(\Omega_{m}\right)}{\sigma_{n}\left(C_{m}^{d}\right)} \underset{n \rightarrow \infty}{\longrightarrow} \frac{\sigma\left(\Omega_{m}\right)}{\sigma\left(C_{m}^{d}\right)} \geqslant 1-\delta(m)
$$


and

$$
\frac{\sigma_{n}^{\prime}\left(S^{-1} \Omega_{m}\right)}{\sigma_{n}^{\prime}\left(C_{m}^{d}\right)} \underset{n \rightarrow \infty}{\longrightarrow} \frac{\sigma\left(S^{-1} \Omega_{m}\right)}{\sigma\left(C_{m}^{d}\right)} \geqslant 1-\delta(m)
$$

where $\delta(m) \rightarrow 0$ as $n \rightarrow \infty$. In particular, $\sigma_{n}\left(\Omega_{m}\right)$ and $\sigma_{n}^{\prime}\left(S^{-1} \Omega_{m}\right)$ are positive if $\delta(m)<1 / 2$ and $n$ is large enough. Hence we can write

This yields

$$
\frac{\sigma_{n}\left(C_{m}^{d}\right)}{\sigma_{n}^{\prime}\left(C_{m}^{d}\right)}=\frac{1+\frac{\sigma_{n}\left(C_{m}^{d} \backslash \Omega_{m}\right)}{\sigma_{n}\left(\Omega_{m}\right)}}{1+\frac{\sigma_{n}^{\prime}\left(C_{m}^{d} \backslash S^{-1} \Omega_{m}\right)}{\sigma_{n}^{\prime}\left(S^{-1} \Omega_{m}\right)}} \underset{n \rightarrow \infty}{\longrightarrow} \frac{1+\frac{\sigma\left(C_{m}^{d} \backslash \Omega_{m}\right)}{\sigma\left(\Omega_{m}\right)}}{1+\frac{\sigma\left(C_{m}^{d} \backslash S^{-1} \Omega_{m}\right)}{\sigma\left(S^{-1} \Omega_{m}\right)}} .
$$

$$
\sigma\left(S^{-1} B\right)=\sigma(B) \frac{1+\frac{\sigma\left(C_{m}^{d} \backslash \Omega_{m}\right)}{\sigma\left(\Omega_{m}\right)}}{1+\frac{\sigma\left(C_{m}^{d} \backslash S^{-1} \Omega_{m}\right)}{\sigma\left(S^{-1} \Omega_{m}\right)}} .
$$

But the above argument is also valid if, at the beginning, we start with $m^{\prime}$ instead of $m$ (and keep the same $m^{\prime}$-box $B$.) This gives

$$
\sigma\left(S^{-1} B\right)=\sigma(B) \frac{1+\frac{\sigma\left(C_{m^{\prime}}^{d} \backslash \Omega_{m^{\prime}}\right)}{\sigma\left(\Omega_{m^{\prime}}\right)}}{1+\frac{\sigma\left(C_{m^{\prime}}^{d} \backslash S^{-1} \Omega_{m^{\prime}}\right)}{\sigma\left(S^{-1} \Omega_{m^{\prime}}\right)}} .
$$

Moreover, as $\sigma\left(C_{m}^{d}\right)>0$, we can choose the $m^{\prime}$-box $B$ in such a way that $\sigma(B)>0$, and comparing the last two equalities, we get

$$
\frac{1+\frac{\sigma\left(C_{m}^{d} \backslash \Omega_{m}\right)}{\sigma\left(\Omega_{m}\right)}}{1+\frac{\sigma\left(C_{m}^{d} \backslash S^{-1} \Omega_{m}\right)}{\sigma\left(S^{-1} \Omega_{m}\right)}}=\frac{1+\frac{\sigma\left(C_{m^{\prime}}^{d} \backslash \Omega_{m^{\prime}}\right)}{\sigma\left(\Omega_{m^{\prime}}\right)}}{1+\frac{\sigma\left(C_{m^{\prime}}^{d} \backslash S^{-1} \Omega_{m^{\prime}}\right)}{\sigma\left(S^{-1} \Omega_{m^{\prime}}\right)}} .
$$

But the ratio on the right-hand side can be made arbitrarily close to 1 by choosing $m^{\prime}$ large enough, hence it is equal to 1 . This proves that, for any $m^{\prime} \geqslant m$ and any $m^{\prime}$-box $B \subset \Omega_{m}, \sigma\left(S^{-1} B\right)=\sigma(B)$. We thus get as in the proof of Lemma 3.2 that $\sigma$ and $S_{*}(\sigma)$ coincide on $\bigcup_{m} \Omega_{m}=X \backslash X_{\mathbf{0}}^{d}$. And since both measures are equal to 0 on $X_{\mathbf{0}}^{d}$, this concludes the proof.

The first two assumptions in Proposition 6.3 will be given by applications of Proposition 5.7 and Proposition 5.9. The following simple example presents the main ideas of how to construct sequences of measures $\left(\sigma_{n}\right)$ and $\left(\sigma_{n}^{\prime}\right)$ satisfying the third requirement of Proposition 6.3.

Example 6.4. - Let $n \notin\left\{n_{\ell}: \ell \geqslant 1\right\}$. Let $J$ be an interval contained in an $n$ crossing, set $J^{\prime}:=J+h_{n}$, assume that $J^{\prime}$ is also contained in an $n$-crossing. Finally, assume that, for each $j \in J,\left\{t_{n}\left(T^{j} x_{i}\right): i=1, \ldots, d\right\}=\{1,2\}$.

Define $\sigma_{n}:=\gamma_{J}$, and $\sigma_{n}^{\prime}:=\gamma_{J^{\prime}}$. For an arbitrary $j \in J$, consider the partition $\{1, \ldots, d\}=G_{0} \sqcup G_{1}$ into two nonempty subsets, where $G_{0}:=\{i \in\{1, \ldots, d\}$ : $\left.t_{n}\left(T^{j} x_{i}\right)=1\right\}$, and $G_{1}:=\left\{i \in\{1, \ldots, d\}: t_{n}\left(T^{j} x_{i}\right)=2\right\}$. Note that, since $J$ is contained in an $n$-crossing, this partition does not depend on the choice of $j \in J$. Let $S_{n}$ be the twisting transformation associated with $\left(G_{0}, G_{1}\right)$. Then for each $m \leqslant n$ and each $m$-box $B \subset \Omega_{m}$, we have $\sigma_{n}^{\prime}\left(S_{n}^{-1} B\right)=\sigma_{n}(B)$.

Indeed, consider first $i \in G_{0}$. Then, for $j \in J, T^{j}\left(x_{i}\right)$ is in the first subcolumn of tower $n$, hence when the orbit of $x_{i}$ reaches the top of tower $n$, it will see no spacer 
before coming back to $C_{n}$. We thus have $j_{n}\left(T^{j+h_{n}} x_{i}\right)=j_{n}\left(T^{j} x_{i}\right)$, and in fact we have the equality $j_{m}\left(T^{j+h_{n}} x_{i}\right)=j_{m}\left(T^{j} x_{i}\right)$ for each $m \leqslant n$ (remember that $j_{n}$ determines $j_{m}$ for $\left.m \leqslant n\right)$.

On the other hand, if $i \in G_{1}$, the orbit of $x_{i}$ will pass through the spacer above the middle subcolumn before coming back to $C_{n}$, and we have, for each $m \leqslant n$, $j_{m}\left(T^{j+h_{n}} x_{i}\right)=j_{m}\left(T^{j} x_{i}\right)-1$ (provided $j_{m}\left(T^{j} x_{i}\right) \neq 0$ ).

Now, if $B \subset \Omega_{m}$ is an $m$-box for some $m \leqslant n$, the above argument shows that, for each $j \in J,\left(T^{\times d}\right)^{j} x \in B \Longleftrightarrow\left(T^{\times d}\right)^{j+h_{n}} x \in S^{-1} B$.

\section{End of the proof of the main result}

Now we come back to the last part of the proof of Theorem 3.10. We interpret Proposition 3.17 as follows:

- either $\sigma$ is a graph measure arising from powers of $T$,

- or there exist infinitely many integers $n$ such that there exists at least one substantial $n$-crossing which is not synchronized.

It only remains to show how this latter property implies that $\sigma$ can be decomposed as a product measure, as explained in Section 3.3 and with the tools of Section 6.

From now on, we thus assume that for infinitely many integers $n$, there exists at least one substantial $n$-crossing which is not synchronized. We have to study different cases, according to the relative positions of these integers $n$ with respect to the sequence $\left(n_{\ell}\right)$.

\subsection{The case $n_{(\ell-1)} \leqslant n \leqslant n_{\ell}-\ell$}

Here we first assume that there exist infinitely many integers $n$ for which

- there exists at least one substantial $n$-crossing which is not synchronized.

- $\exists \ell: n_{(\ell-1)} \leqslant n \leqslant n_{\ell}-\ell$.

Let us consider such an $n$. To unify the treatments of the cases $n=n_{(\ell-1)}$ and $n_{(\ell-1)}<n \leqslant n_{\ell}-\ell$, we set

$$
\tilde{h}_{n}:= \begin{cases}h_{n}+h_{n(\ell-1)-k(\ell-1)} & \text { if } n=n_{(\ell-1)} \\ h_{n} & \text { if } n_{(\ell-1)}<n \leqslant n_{\ell}-\ell .\end{cases}
$$

In this way, as long as we stay inside the interval $\left[-100 h_{n}, 100 h_{n}\right]$ (which is contained

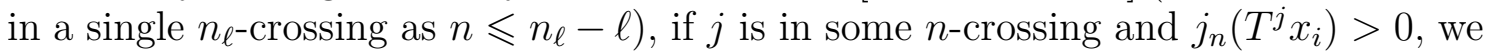
have

$$
j_{n}\left(T^{j+\tilde{h}_{n}} x_{i}\right)= \begin{cases}j_{n}\left(T^{j} x_{i}\right) & \text { if } t_{n}\left(T^{j} x_{i}\right)=1, \\ j_{n}\left(T^{j} x_{i}\right)-1 & \text { if } t_{n}\left(T^{j} x_{i}\right)=2, \\ \text { one or other of the above values } & \text { if } t_{n}\left(T^{j} x_{i}\right)=3 .\end{cases}
$$

Let $J$ be a substantial $n$-crossing which is not synchronized. Then for $j \in J$, $\left\{t_{n}\left(T^{j} x_{i}\right): i=1, \ldots, d\right\}$ contains at least two different values (which do not depend on the choice of $j \in J$ since $j \mapsto t_{n}\left(T^{j} x_{i}\right)$ is constant on an $n$-crossing). 
We first assume that $\{1,2\} \subset\left\{t_{n}\left(T^{j} x_{i}\right): i=1, \ldots, d\right\}$. Then, by the above formula, for $j \in J \backslash \min J$, the difference $j_{n}\left(T^{j} x_{i}\right)-j_{n}\left(T^{j+\tilde{h}_{n}} x_{i}\right)$ takes both values 0 and 1 as $i$ runs over $\{1, \ldots, d\}$. Set, for $a=0,1$

$$
G_{a}:=\left\{i: j_{n}\left(T^{j} x_{i}\right)-j_{n}\left(T^{j+\tilde{h}_{n}} x_{i}\right)=a\right\} .
$$

Then we can define a twisting transformation $S_{n}$ with this partition. We also define the interval $J^{\prime}:=J+\tilde{h}_{n}$, and the two measures $\sigma_{n}:=\gamma_{J}, \sigma_{n}^{\prime}:=\gamma_{J^{\prime}}$.

As explained in Example 6.4, if for some $B$ is an $m$-box for some $m \leqslant n$ with $B \subset \Omega_{m}$, we then have

$$
\sigma_{n}^{\prime}(B)=\sigma_{n}\left(S_{n}^{-1}(B)\right)
$$

Let us explain how we construct $S_{n}, \sigma_{n}$ and $\sigma_{n}^{\prime}$ when $\{2,3\}=\left\{t_{n}\left(T^{j} x_{i}\right): i=\right.$ $1, \ldots, d\}$ for $j \in J$. Then, for $j \in J \backslash\{\max J\}$, the difference $j_{n}\left(T^{j-\tilde{h}_{n}} x_{i}\right)-j_{n}\left(T^{j} x_{i}\right)$ takes both values 0 and 1 as $i$ runs over $\{1, \ldots, d\}$. In this case we define the partition by

$$
G_{a}:=\left\{i: j_{n}\left(T^{j-\tilde{h}_{n}} x_{i}\right)-j_{n}\left(T^{j} x_{i}\right)=a\right\}, \quad a=0,1,
$$

and the corresponding twisting transformation $S_{n}$. We consider $J^{\prime}:=J-\tilde{h}_{n}, \sigma_{n}:=\gamma_{J^{\prime}}$ and $\sigma_{n}^{\prime}:=\gamma_{J}$, and we get (7.1) for any $m$-box $B \subset \Omega_{m}, m \leqslant n$.

Finally we consider the case when $\{1,3\}=\left\{t_{n}\left(T^{j} x_{i}\right): i=1, \ldots, d\right\}$ for $j \in J$. Then, for $j \in J \backslash\{\min J\}$, there are two options:

- either there exists $i \in\{1, \ldots, d\}$ with $t_{n}\left(T^{j} x_{i}\right)=3$, and $j_{n}\left(T^{j} x_{i}\right)-$ $j_{n}\left(T^{j+\tilde{h}_{n}} x_{i}\right)=1$ (we see one spacer above the third column for at least one coordinate),

- or for each $i \in\{1, \ldots, d\}$ such that $t_{n}\left(T^{j} x_{i}\right)=3$, we have $j_{n}\left(T^{j} x_{i}\right)$ $j_{n}\left(T^{j+\tilde{h}_{n}} x_{i}\right)=0$ (we see no spacer above the third column).

In the first option, we do the same construction as in the case $\{1,2\} \subset\left\{t_{n}\left(T^{j} x_{i}\right)\right.$ : $i=1, \ldots, d\}$. In the second option, we observe that

$$
j_{n}\left(T^{j} x_{i}\right)-j_{n}\left(T^{j+2 \tilde{h}_{n}} x_{i}\right)= \begin{cases}1 & \text { if } t_{n}\left(T^{j} x_{i}\right)=1 \\ 0 & \text { if } t_{n}\left(T^{j} x_{i}\right)=3\end{cases}
$$

We then set $J^{\prime}:=J+2 \tilde{h}_{n}$, and construct $S_{n}, \sigma_{n}$ and $\sigma_{n}^{\prime}$ as before.

Since we assume that there are infinitely many integers $n$ with these properties, we can apply Proposition 5.7 to prove that $\sigma_{n} \rightarrow \sigma$ and $\sigma_{n}^{\prime} \rightarrow \sigma$. Indeed, $J$ and $J^{\prime}$ are both contained in $\left\{-5 h_{n}, \ldots, 5 h_{n}\right\}$ which is contained in an $n_{\ell}$-crossing. Since $J$ is a substantial $n$-crossing, we have $\eta h_{n} \leqslant|J|=\left|J^{\prime}\right| \leqslant h_{n}$, and we have $\gamma_{\left\{-5 h_{n}, \ldots, 5 h_{n}\right\}} \underset{n \rightarrow \infty}{\longrightarrow} \sigma$. Then Proposition 6.3 shows that $\sigma$ can be decomposed as a product of two Radon measures to which we can apply the induction hypothesis.

We are now reduced to study the case where, for each $\ell$ large enough and each $n_{\ell-1} \leqslant n \leqslant n_{\ell}-\ell$, all substantial $n$-crossings are synchronized, but still there exist infinitely many integers $n$ for which at least one substantial $n$-crossing is not synchronized. 


\subsection{The case $n_{\ell}-\ell<n<n_{\ell}-k(\ell)$}

This case cannot be treated as the preceding one since, for such an $n$, we are not sure any more that an interval around 0 and of size of order $h_{n}$ is completely contained in an $n_{\ell}$-crossing. Hence on such an interval, when the orbit of some $x_{i}$ leaves $C_{n}$, it may stay out of $C_{n}$ for a long time (up to $h_{n_{\ell}-k(\ell)}+1$, which may be much larger than $h_{n}$ ).

The following lemma is introduced to remedy this problem.

Lemma 7.1. - For each large enough $\ell$, there exists an integer

$$
n_{\text {good }}(\ell) \in\left\{n_{\ell}-k(\ell)+p_{1}+2 p_{2}, \ldots, n_{\ell}-k(\ell)+p_{1}+2 p_{2}+d\right\}
$$

such that $\left\{h_{n_{\text {good }}(\ell)}, \ldots, 2 h_{n_{\text {good }}(\ell)}\right\}$ is contained in an $n_{\ell}$-crossing.

Proof. - Assume that $\ell>\max _{i} \ell\left(x_{i}\right)$, and that $n_{\ell}-k(\ell)+p_{1}+2 p_{2}+d<n_{\ell}$. We say that the coordinate $i \in\{1, \ldots, d\}$ is bad for $n$ if there exists some $j \in\left\{h_{n}, \ldots, 2 h_{n}\right\}$ such that $T^{j} x_{i} \notin C_{n_{\ell}}$. We observe that if $\left\{h_{n}, \ldots, 2 h_{n}\right\}$ is not contained in an $n_{\ell}$-crossing, then at least one coordinate is bad for $n$. To prove the lemma, it is sufficient to show that for each $i=1, \ldots, d$, there is at most one $n \in\left\{n_{\ell}-k(\ell)+p_{1}+\right.$ $\left.2 p_{2}, \ldots, n_{\ell}-k(\ell)+p_{1}+2 p_{2}+d\right\}$ for which $i$ is bad. So assume that $i$ is bad for some $n$ in this interval, and let $j \in\left\{h_{n}, \ldots, 2 h_{n}\right\}$ such that $T^{j} x_{i} \notin C_{n_{\ell}}$. The orbit of $x_{i}$ comes back to $C_{n_{\ell}}$ before $j+h_{n_{\ell}-k(\ell)}+1$, then stays in $C_{n_{\ell}}$ on an interval of length $h_{n_{\ell}}$. But we have $j+h_{n_{\ell}-k(\ell)}+1<h_{n+1}$ and $j+h_{n_{\ell}}>2 h_{n_{\ell}-k(\ell)+p_{1}+2 p_{2}+d}$, hence $i$ cannot be bad for any $n^{\prime}>n$ in the interval $\left\{n_{\ell}-k(\ell)+p_{1}+2 p_{2}, \ldots, n_{\ell}-k(\ell)+p_{1}+2 p_{2}+d\right\}$.

Remark 7.2. - It follows from Proposition 5.7 that we have the following convergence:

$$
\gamma_{\left\{h_{n_{\text {good }}(\ell)}, \ldots, 2 h_{n_{\text {good }}(\ell)}\right\} \underset{\ell \rightarrow \infty}{\longrightarrow} \sigma .}
$$

Indeed, this proposition applies where $\ell$ plays the role of $\ell_{m}-1, n_{\text {good }}(\ell)$ is $n(m)$, $\left\{h_{n_{\text {good }}(\ell)}, \ldots, 2 h_{n_{\text {good }}(\ell)}\right\}$ is $J_{m}$, and $\left\{0, \ldots, 2 h_{n_{\text {good }}(\ell)}\right\}$ is $I_{m}$.

We will also need the following result, which will also be useful in the next section. We consider here an integer $n$ such that $n_{\ell}-\ell<n \leqslant n_{\ell}-k(\ell)$ for some $\ell$, and we set $n^{\prime}:=n_{\ell}-k(\ell)$. As in the proof of Lemma 5.1 we introduce the fake $n^{\prime}$-tower, and the fake $n$-tower that mimicks the structure of tower $n$ inside tower $n^{\prime}$. (Note that this is possible as long as $n \leqslant n^{\prime}$.) $\tilde{C}_{n}$ is the union of the levels of the fake $n$-tower, and $\bar{C}_{n}:=C_{n} \sqcup \tilde{C}_{n}$. Recall that $j_{n}$ indicates the level of tower $n$ to which a point in $C_{n}$ belongs. We extend this definition to points in $\bar{C}_{n}: \bar{j}_{n}$ indicates the level of tower $n$ (possibly fake) to which a point in $\bar{C}_{n}$ belongs.

Lemma 7.3. - For each large enough $\ell$, for each $n$ such that $n_{\ell}-\ell<n \leqslant n_{\ell}-k(\ell)$, for each integer $r$ such that $\left|r h_{n}\right|<10 h_{n_{\ell}}$, for each $i=1, \ldots, d$, we have

- $x_{i} \in C_{n}$ and $4^{\ell}<j_{n}\left(x_{i}\right)<h_{n}-1-4^{\ell}$,

- $T^{r h_{n}} x_{i} \in \bar{C}_{n}$,

- $j_{n}\left(x_{i}\right)-4^{\ell} \leqslant \bar{j}_{n}\left(T^{r h_{n}} x_{i}\right) \leqslant j_{n}\left(x_{i}\right)+4^{\ell}$. 
Proof. - If $\ell-1 \geqslant \max _{i} \ell\left(x_{i}\right)$ (cf. Lemma 2.4), we have $x_{i} \in C_{n_{(\ell-2)}} \subset C_{n}$ for $i=1, \ldots, d$. Moreover, $x_{i}$ is not in the first hundred occurrences of tower $n_{(\ell-1)}-(\ell-1)$ inside tower $n_{(\ell-1)}$. Hence, as $n_{\ell} / \ell \rightarrow \infty$ as $\ell \rightarrow \infty$, and remembering (2.5), we have for $\ell$ large enough

$$
j_{n}\left(x_{i}\right) \geqslant j_{n_{(\ell-1)}}\left(x_{i}\right) \geqslant 100 h_{n_{(\ell-1)}-(\ell-1)}>100 \times 3^{n_{(\ell-1)}-(\ell-1)}>100 \times 3^{n_{(\ell-2)}}>4^{\ell} .
$$

By a symmetric argument, we also get for $\ell$ large enough $j_{n}\left(x_{i}\right)<h_{n}-1-4^{\ell}$.

We observe that, since $\left|r h_{n}\right|<10 h_{n_{\ell}},\left\{-\left|r h_{n}\right|, \ldots,\left|r h_{n}\right|\right\}$ is contained in an $n_{(\ell+1)^{-}}$ crossing. Hence when the orbit of some coordinate leaves $C_{n}$ on this interval, it comes back after $0,1, h_{n^{\prime}}$ or $h_{n^{\prime}}+1$ iterations of the transformation. If we consider the enlarged tower $\bar{C}_{n}$ instead of $C_{n}$, then $T^{j} x_{i}$ comes back to $\bar{C}_{n}$ after 0 or 1 iteration of the transformation. Hence $\bar{j}_{n}\left(T^{h_{n}} x_{i}\right) \in\left\{j_{n}\left(x_{i}\right)-1, j_{n}\left(x_{i}\right)\right\}$, and by a simple induction we get $\bar{j}_{n}\left(T^{r h_{n}} x_{i}\right) \in\left\{j_{n}\left(x_{i}\right)-|r|, \ldots, j_{n}\left(x_{i}\right)+|r|\right\}$. The result then follows from the fact that $|r|<4^{\ell}$ (indeed, by hypothesis we have $n>n_{\ell}-\ell$, hence $h_{n}>10 h_{n_{\ell}} / 4^{\ell}$ for $\ell$ large enough).

Remark 7.4. - If, as in the case we are currently studying, we have the strict inequality $n<n_{\ell}-k(\ell)$, then the number of occurrences of the fake $n$-tower inside the fake $n^{\prime}$-tower is a multiple of 3 . So we can extend the function $t_{n}$ to a function $\bar{t}_{n}$ defined on $\bar{C}_{n}$ in such a way that, for each $r$ such that $\left|r h_{n}\right|<h_{n_{\ell}}$ and each $i=1, \ldots, d$

$$
\bar{t}_{n}\left(T^{r h_{n}} x_{i}\right)=\bar{t}_{n}\left(x_{i}\right)+r \bmod 3 .
$$

We consider now an integer $n$ with $n_{\ell}-\ell<n<n_{\ell}-k(\ell)$ for some $\ell$, where $\ell$ is large enough to apply the preceding lemmas, and we assume that there is at least one substantial $n$-crossing which is not synchronized. With the assumption stated at the end of Section 7.1, we can also assume that for each $n_{(\ell-1)} \leqslant m \leqslant n-1$, all substantial $m$-crossings are synchronized. Then, as in the second part of the proof of Proposition 3.17, we can construct inductively a family $\left(J_{m}\right)_{n_{(\ell-1)} \leqslant m \leqslant n}$ where

- $J_{n_{(\ell-1)}}$ is a substantial $n$-crossing of length $\geqslant(1-(d+2) \eta) h_{n_{(\ell-1)}}$;

- for each $m>n_{(\ell-1)}, J_{m}$ is a substantial $m$-crossing extending $J_{m-1}$ and of size $\left|J_{m}\right| \geqslant h_{m}-(d+2) \eta h_{n_{(\ell-1)}}$ (see Remark 3.18).

In particular, the size of the $n$-crossing $J_{n}$ satisfies $\left|J_{n}\right| \geqslant h_{n}-(d+2) \eta h_{n_{(\ell-1)}}$, and we can assume that $\ell$ is large enough so that this implies $\left|J_{n}\right| \geqslant(1-\eta / 100) h_{n}$. Since we assume that there is at least one substantial $n$-crossing which is not synchronized, this ensures that $J_{n}$ itself is not synchronized. Indeed, assume that there is another substantial $n$-crossing $J_{n}^{\prime}$ which is not synchronized. Because the length of $J_{n}$ is so close to $h_{n}$, the orbit of each coordinate has to pass through the top of tower $n$ between $J_{n}$ and the other substantial $n$-crossing $J_{n}^{\prime}$. But $J_{n}^{\prime}$ intersects $\boldsymbol{I}_{n}$, hence the distance between $J_{n}$ and $J_{n}^{\prime}$ is less than $h_{n}$. This shows that, for each $i=1, \ldots, d$, $t_{n}\left(x_{i}\right)$ increases by $1 \bmod 3$ between the two substantial $n$-crossings. Then, as $J_{n}^{\prime}$ is not synchronized, $J_{n}$ itself is not synchronized.

Moreover, by Remark 2.6, the $\left(n_{\ell}-\ell\right)$-crossing containing 0 covers the interval $\left\{-100 h_{n_{(\ell-1)}}, \ldots, 100 h_{n_{(\ell-1)}}\right\}$. In particular, it contains $J_{(\ell-1)}$ hence it is $J_{\left(n_{\ell}-\ell\right)}$. As $J_{n}$ extends $J_{\left(n_{\ell}-\ell\right)}$, this proves that $J_{n}$ contains 0 . 
Consider the set

$$
R:=\left\{r \geqslant 1: J_{n}+r h_{n} \subset\left\{h_{n_{\text {good }}(\ell)}, \ldots, 2 h_{n_{\text {good }}(\ell)}\right\}\right\}
$$

Since $2 h_{n_{\text {good }}(\ell)}<h_{n_{\ell}}$, Lemma 7.3 applies to each $r \in R$. In particular, for each $r \in R$ and each $i=1, \ldots, d$ we have $T^{r h_{n}} x_{i} \in \bar{C}_{n}$. But by choice of $n_{\text {good }}(\ell)$, we also know that $T^{r h_{n}} x_{i} \in C_{n_{\ell}}$. Since $C_{n_{\ell}}$ is disjoint from the fake $n^{\prime}$-tower, $T^{r h_{n}} x_{i} \notin \tilde{C}_{n}$, and finally $T^{r h_{n}} x_{i} \in C_{n}$. Let $\tilde{J}_{n}$ be the interval obtained by removing the first $4^{\ell}$ elements of the $n$-crossing $J_{n}$. Then, by Lemma 7.3 , we have $0 \in \widetilde{J}_{n}$, and for each $r \in R, \tilde{J}_{n}+r h_{n}$ is contained in an $n$-crossing. Note that the size of $\tilde{J}_{n}$ is $\geqslant(1-\eta / 100) h_{n}-4^{\ell}>(1-\eta) h_{n}$.

By Remark 7.4, for each $r \in R$ and each $i=1, \ldots, d$, we have $t_{n}\left(T^{r h_{n}} x_{i}\right)=$ $t_{n}\left(x_{i}\right)+r \bmod 3$. In particular, as $J_{n}$ is not synchronized, for each $r \in R, t_{n}\left(T^{r h_{n}} x_{i}\right)$ takes at least 2 values as $i$ varies. We define

$$
r_{0}:=\min \left\{r \in R: t_{n}\left(T^{r h_{n}} x_{i}\right) \text { takes both values } 1 \text { and } 2 \text { as } i=1, \ldots, d\right\} .
$$

We have $\min R \leqslant r_{0} \leqslant \min R+2$.

Now let us consider $r$ such that both $r$ and $r+1$ are in $R$. For $j \in \tilde{J}_{n}$, we want to compare the position in tower $n$ of $T^{j+r h_{n}} x_{i}$ and $T^{j+(r+1) h_{n}} x_{i}$ for each coordinate.

- If $i$ is such that $t_{n}\left(T^{r h_{n}} x_{i}\right)=1$, the orbit of $x_{i}$ will not pass through a spacer between $\tilde{J}_{n}+r h_{n}$ and $\tilde{J}_{n}+(r+1) h_{n}$. Hence in this case we have $j_{n}\left(T^{j+r h_{n}} x_{i}\right)-j_{n}\left(T^{j+(r+1) h_{n}} x_{i}\right)=0$.

- If $i$ is such that $t_{n}\left(T^{r h_{n}} x_{i}\right)=2$, the orbit of $x_{i}$ will pass through one spacer between $\tilde{J}_{n}+r h_{n}$ and $\tilde{J}_{n}+(r+1) h_{n}$. Hence in this case we have $j_{n}\left(T^{j+r h_{n}} x_{i}\right)-$ $j_{n}\left(T^{j+(r+1) h_{n}} x_{i}\right)=1$.

- If $i$ is such that $t_{n}\left(T^{r h_{n}} x_{i}\right)=3$, we have $j_{n}\left(T^{j+r h_{n}} x_{i}\right)-j_{n}\left(T^{j+(r+1) h_{n}} x_{i}\right) \in$ $\{0,1\}$, depending on the position of $T^{r h_{n}} x_{i}$ in the subsequent towers.

More precisely, in every case the value of $j_{n}\left(T^{j+r h_{n}} x_{i}\right)-j_{n}\left(T^{j+(r+1) h_{n}} x_{i}\right)$ is determined as follows: let $m$ be the smallest integer, $m \geqslant 0$, such that $t_{n+m}\left(T^{r h_{n}} x_{i}\right) \neq 3$. Note that $n+m<n_{\ell}$ since $\tilde{J}_{n}+r h_{n}$ and $\tilde{J}_{n}+(r+1) h_{n}$ are contained in the same $n_{\ell}$-crossing. Then we have

$$
j_{n}\left(T^{j+r h_{n}} x_{i}\right)-j_{n}\left(T^{j+(r+1) h_{n}} x_{i}\right)= \begin{cases}0 & \text { if } t_{n+m}\left(T^{r h_{n}} x_{i}\right)=0 \\ 1 & \text { if } t_{n+m}\left(T^{r h_{n}} x_{i}\right)=1 .\end{cases}
$$

The difficulty which arises here is that, when $t_{n}\left(x_{i}\right)=3$, the value of this difference may vary with $r$. This is why we need the following lemma.

Lemma 7.5. - There exists an integer $s, 0 \leqslant s<3^{d-1}$, such that

- $s=0 \bmod 3$,

- for each $i=1, \ldots, d$, there exists a smaller integer $m_{i}, 0 \leqslant m_{i} \leqslant d-2$, satisfying $t_{n+m_{i}}\left(T^{\left(r_{0}+s\right) h_{n}} x_{i}\right) \neq 3$.

Proof. - We first remark that for each $i=1, \ldots, d$ and each $m \geqslant 0$, the map $r \in R \mapsto t_{n+m}\left(T^{r h_{n}} x_{i}\right)$ has a very regular behaviour. Indeed, it is constant on intervals of length $3^{m}$, and if both $r$ and $r+3^{m}$ are in $R$, we have

$$
t_{n+m}\left(T^{\left(r+3^{m}\right) h_{n}} x_{i}\right)=t_{n+m}\left(T^{r h_{n}} x_{i}\right)+1 \bmod 3 .
$$


If $\left\{i \in\{1, \ldots, d\}: t_{n}\left(T^{r_{0} h_{n}} x_{i}\right)=3\right\}=\emptyset$, we just have to set $s:=0$ and we get the result with $m_{i}=0$ for each $i$. Otherwise, we consider

$$
i_{1}:=\min \left\{i \in\{1, \ldots, d\}: t_{n}\left(T^{r_{0} h_{n}} x_{i}\right)=3\right\} .
$$

Then we choose $s_{1} \in\{0,1,2\}$ such that $t_{n+1}\left(T^{\left(r_{0}+3 s_{1}\right) h_{n}} x_{i_{1}}\right)=1$, which is possible by (7.3). We note that replacing $r_{0}$ by $\left(r_{0}+3 s_{1}\right)$ does not affect the values of the $t_{n}\left(T^{r h_{n}} x_{i}\right)$. Now, if $\left\{i \in\{1, \ldots, d\}: t_{n+1}\left(T^{\left(r_{0}+3 s_{1}\right) h_{n}} x_{i}\right)=3\right\}=\emptyset$, we have the result with $s=3 s_{1}$. Otherwise, we set

$$
i_{2}:=\min \left\{i \in\{1, \ldots, d\}: t_{n+1}\left(T^{\left(r_{0}+3 s_{1}\right) h_{n}} x_{i}\right)=3\right\} .
$$

(Note that $i_{2}>i_{1}$.) Then we choose $s_{2} \in\{0,1,2\}$ such that

$$
t_{n+2}\left(T^{\left(r_{0}+3 s_{1}+9 s_{2}\right) h_{n}} x_{i_{2}}\right)=1 .
$$

Again, replacing $\left(r_{0}+3 s_{1}\right)$ by $\left(r_{0}+3 s_{1}+9 s_{2}\right)$ does not affect the values of the $t_{n+m}\left(T^{r h_{n}} x_{i}\right), m=0,1$.

We continue in this way until we have found $s_{1}, \ldots, s_{k} \in\{0,1,2\}$ such that, for each $i=1, \ldots, d$, there exists $m, 0 \leqslant m \leqslant k$ such that

$$
t_{n+m}\left(T^{\left(r_{0}+3 s_{1}+\cdots+3^{k} s_{k}\right) h_{n}} x_{i}\right) \neq 3 .
$$

Since the algorithm also produces an strictly increasing sequence $i_{1}<i_{2}<\cdots$ in $\{1, \ldots, d\}$, we are guaranteed that it will stop in $k \leqslant d$ steps. Moreover, since the sequence $i_{1}<\cdots<i_{k}$ contains no $i$ such that $t_{n}\left(T^{r_{0} h_{n}} x_{i}\right) \in\{1,2\}$, we have in fact $k \leqslant d-2$. We then get the announced result by setting $s:=3 s_{1}+\cdots+3^{k} s_{k} \leqslant 3^{d-1}$.

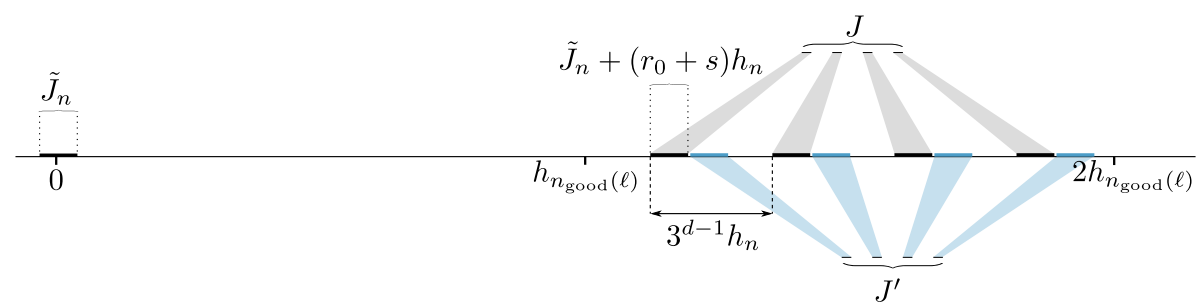

Figure 7.1. The choice of $J$ and $J^{\prime}$ when $n_{\ell}-\ell<n<n_{\ell}-k(\ell)$

Now, with $s$ defined in Lemma 7.5, we set

$$
R_{1}:=\left\{r \in R:(r+1) \in R \text { and } r=r_{0}+s \bmod 3^{d-1}\right\} .
$$

Observe that $R_{1} \neq \emptyset$, as $R$ is an interval of size

$$
|R| \geqslant\left\lfloor h_{n_{\text {good }}(\ell} / h_{n}\right\rfloor \geqslant 3^{n_{\text {good }}(\ell)-n} \geqslant 3^{p_{1}} \geqslant 3^{d} .
$$

Recall that for each $0 \leqslant m \leqslant d-2$, and each $i=1, \ldots, d$, the map $r \in R \mapsto$ $t_{n+m}\left(T^{r h_{n}} x_{i}\right)$ is $3^{d-1}$-periodic. Hence, by choice of $s$, for each $i=1, \ldots, d$ the difference

$$
j_{n}\left(T^{j+r h_{n}} x_{i}\right)-j_{n}\left(T^{j+(r+1) h_{n}} x_{i}\right)
$$


depends neither on $j \in \tilde{J}_{n}$ nor on $r \in R_{1}$. Moreover, by choice of $r_{0}$, this difference takes both values 0 and 1 as $i$ varies. Therefore we can construct the following partition $\{1, \ldots, d\}=G_{0} \sqcup G_{1}$, where for $a=0,1$,

$$
G_{a}:=\left\{i: \forall j \in \tilde{J}_{n}, \forall r \in R_{1}, j_{n}\left(T^{j+r h_{n}} x_{i}\right)-j_{n}\left(T^{j+(r+1) h_{n}} x_{i}\right)=a\right\},
$$

Then we denote by $S_{n}$ the corresponding twisting transformation. We also consider the two disjoint subsets $J$ and $J^{\prime}$ of $\left\{h_{n_{\text {good }}(\ell)}, \ldots, 2 h_{n_{\text {good }}(\ell)}\right\}$ defined by

$$
J:=\bigsqcup_{r \in R_{1}} \tilde{J}_{n}+r h_{n}, \quad \text { and } J^{\prime}:=J+h_{n}
$$

(See Figure 7.1) Then, as in Example 6.4, the measures $\sigma_{n}:=\gamma_{J}$ and $\sigma_{n}^{\prime}:=\gamma_{J^{\prime}}$ satisfy (7.1) for each $m$-box $B \subset \Omega_{m}, m \leqslant n$.

Assuming the existence of infinitely many integers $n$ with these properties, we can apply Proposition 5.9 to prove that $\sigma_{n} \rightarrow \sigma$ and $\sigma_{n}^{\prime} \rightarrow \sigma$. Indeed, $J$ and $J^{\prime}$ are both contained in $\left\{h_{n_{\operatorname{good}}(\ell)}, \ldots, 2 h_{n_{\operatorname{good}}(\ell)}\right\}$ which is contained in an $n_{\ell^{-}}$-crossing. They both have the structure required in the assumptions of this proposition, with $M=3^{d-1}$. Moreover, we also know by Remark 7.2 that $\gamma_{\left\{h_{n_{\text {good }}(\ell)}, \ldots, 2 h_{n_{\text {good }}(\ell)}\right\}} \underset{n \rightarrow \infty}{\longrightarrow} \sigma$.

Then Proposition 6.3 shows that $\sigma$ can be decomposed as a product of two Radon measures to which we can apply the induction hypothesis.

We are now reduced to study the case where, for each $\ell$ large enough and each $n_{\ell-1} \leqslant n<n_{\ell}-k(\ell)$, all substantial $n$-crossings are synchronized, but still there exist infinitely many integers $n$ for which at least one substantial $n$-crossing is not synchronized.

\subsection{The case $n=n_{\ell}-k(\ell)$}

We consider now an integer $n$ of the form $n=n_{\ell}-k(\ell)$ for some $\ell$, where $\ell$ is large enough. We assume that there is at least one substantial $n$-crossing which is not synchronized, and also that for each $n_{(\ell-1)} \leqslant m \leqslant n-1$, all substantial $m$-crossings are synchronized. Then, as in Section 7.2 , we prove that the $n$-crossing $J_{n}$ containing 0 is of size $\left|J_{n}\right| \geqslant(1-\eta / 100) h_{n}$, and is not synchronized. We also define $\tilde{J}_{n} \subset J_{n}$ as in the previous section: $\tilde{J}_{n}$ contains 0 and $\left|\tilde{J}_{n}\right| \geqslant(1-\eta) h_{n}$.

We still work with the fake tower $n$, as introduced before Lemma 7.3 which is still valid in this case. The new difficulty here is that we cannot anymore extend $t_{n}$ to $\bar{C}_{n}$.

We consider integers $r$ with $0 \leqslant r \leqslant 10 d$, and we assume that $\ell$ is large enough so that $k(\ell)>10 d$, thus $10 d<h_{n_{\ell}} / h_{n}$ and the results of Lemma 7.3 are valid for these integers $r$. In particular, for each such $r$, either $\tilde{J}_{n}+r h_{n}$ is contained in an $n$-crossing (we then say that $r$ corresponds to a true $n$-crossing), or there is one coordinate $x_{i}$ such that $T^{r h_{n}} x_{i}$ is in the fake tower $n \tilde{C}_{n}$ (in this case we say that $r$ corresponds to a fake $n$-crossing). Observe that for each $i=1, \ldots, d$, there is at most one integer $r, 0 \leqslant r<\left\lfloor h_{n_{\ell}} / h_{n}\right\rfloor$, such that $T^{r h_{n}} x_{i}$ is in the fake tower $n$. Indeed, as everything takes place in a single $C_{n_{(\ell+1)}}$-crossing, when the orbit of $x_{i}$ leaves $C_{n_{\ell}}$, it comes back to $C_{n_{\ell}}$ after at most $h_{n}+1$ units of time, and then stays in $C_{n_{\ell}}$ for $h_{n_{\ell}}$ units of time. 
If both $T^{r h_{n}} x_{i}$ and $T^{(r+1) h_{n}} x_{i}$ are in $C_{n}$, then $t_{n}\left(T^{(r+1) h_{n}} x_{i}\right)=t_{n}\left(T^{r h_{n}} x_{i}\right)+1 \bmod 3$. If $T^{r h_{n}} x_{i}$ is in the fake tower $n$, then $T^{(r-1) h_{n}} x_{i}$ and $T^{(r+1) h_{n}} x_{i}$ are in $C_{n}$, and we have $t_{n}\left(T^{(r-1) h_{n}} x_{i}\right)=3$, and $t_{n}\left(T^{(r+1) h_{n}} x_{i}\right)=1$.

With these facts in mind, we can prove the following lemma.

Lemma 7.6. - There exist two consecutive integers, $-2 \leqslant r<r+1 \leqslant 10 d$, such that

- $\tilde{J}_{n}+r h_{n}$ is contained in an $n$-crossing,

- $\tilde{J}_{n}+(r+1) h_{n}$ is contained in an $n$-crossing,

- $\left\{i \in\{1, \ldots, d\}: t_{n}\left(T^{r h_{n}} x_{i}\right)=1\right\} \neq \emptyset$,

- $\left\{i \in\{1, \ldots, d\}: t_{n}\left(T^{r h_{n}} x_{i}\right)=2\right\} \neq \emptyset$.

Proof. - There is at most $d$ integers $r, 0 \leqslant r \leqslant 10 d$, such that $\tilde{J}_{n}+r h_{n}$ corresponds to a fake $n$-crossing (indeed, each coordinate can be responsible for only one $r$ for which this property fails). Hence there is a smaller integer $r_{0}, 0 \leqslant r_{0} \leqslant 10 d-2$, such that $r_{0},\left(r_{0}+1\right),\left(r_{0}+2\right)$ and $\left(r_{0}+3\right)$ correspond to true $n$-crossings.

If $r_{0}=0$, since $J_{n}$ is not synchronized, there are two coordinates $x_{i_{1}}$ and $x_{i_{2}}$ such that $t_{n}\left(x_{i_{1}}\right) \neq t_{n}\left(x_{i_{2}}\right)$. If $\left\{t_{n}\left(x_{i_{1}}\right), t_{n}\left(x_{i_{2}}\right)\right\}=\{1,2\}$, we just have to take $r=0$. If $\left\{t_{n}\left(x_{i_{1}}\right), t_{n}\left(x_{i_{2}}\right)\right\}=\{1,3\}$, we set $r=1$, and if $\left\{t_{n}\left(x_{i_{1}}\right), t_{n}\left(x_{i_{2}}\right)\right\}=\{2,3\}$, we set $r=2$. In all these cases we get

$$
\left\{t_{n}\left(T^{r h_{n}} x_{i_{1}}\right), t_{n}\left(T^{r h_{n}} x_{i_{2}}\right)\right\}=\{1,2\}
$$

If $r_{0}>0$ and the $n$-crossing containing $r_{0} h_{n}$ is not synchronized, then we can proceed as in the previous case, replacing 0 by $r_{0}$.

If $r_{0}>0$ and the $n$-crossing containing $r_{0} h_{n}$ is synchronized, then by definition of $r_{0},\left(r_{0}-1\right)$ corresponds to a fake $n$-crossing, hence there is at least one coordinate $x_{i_{1}}$ such that $t_{n}\left(T^{r_{0} h_{n}} x_{i_{1}}\right)=1$. Since the corresponding $n$-crossing is assumed to be synchronized, we have $t_{n}\left(T^{r_{0} h_{n}} x_{i}\right)=1$ for each $i=1, \ldots, d$. We also observe that there exists at least one coordinate $x_{i_{2}}$ such that $T^{\left(r_{0}-1\right) h_{n}} x_{i_{2}} \in C_{n}$. Indeed, otherwise all coordinates would be in the fake $n$-tower at the same time, and this would imply that the $n$-crossing $J_{n}$ containing 0 is synchronized. Now we take $r:=r_{0}-3$. Then for each coordinate $x_{i}$ such that $T^{\left(r_{0}-1\right) h_{n}} x_{i} \in C_{n}$, we have $t_{n}\left(T^{r h_{n}} x_{i}\right)=1$, and for each coordinate $x_{i}$ such that $T^{\left(r_{0}-1\right) h_{n}} x_{i} \notin C_{n}$, we have $t_{n}\left(T^{r h_{n}} x_{i}\right)=2$.

Now, with $r$ provided by Lemma 7.6, we consider the two measures $\sigma_{n}:=\gamma_{\tilde{J}_{n}+r h_{n}}$ and $\sigma_{n}^{\prime}:=\gamma_{\tilde{J}_{n}+(r+1) h_{n}}$ (see Figure 7.2). We can show by the same argument as in Section 7.1 that there exists a twisting transformation $S_{n}$ such that (7.1) holds whenever $B$ is an $m$-box in $\Omega_{m}$ for some $m \leqslant n$.

Finally, if we have infinitely many integers $n$ to which the above arguments apply, Proposition 5.7 shows that $\sigma_{n} \underset{n \rightarrow \infty}{\longrightarrow} \sigma$ and $\sigma_{n}^{\prime} \underset{n \rightarrow \infty}{\longrightarrow} \sigma$. Then Proposition 6.3 shows that $\sigma$ can be decomposed as a product of two Radon measures to which we can apply the induction hypothesis. 


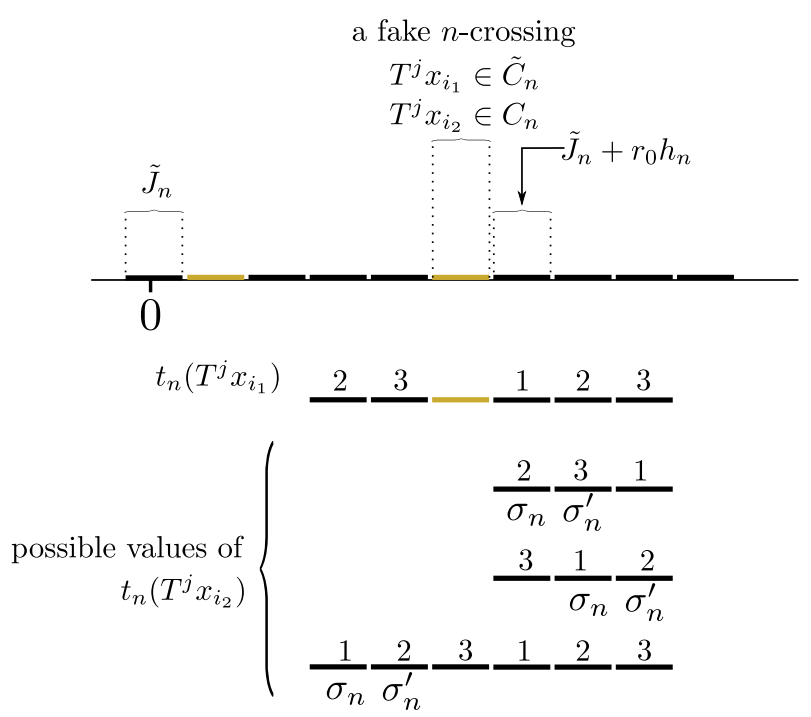

Figure 7.2. The three possible cases for the choice of the measures $\sigma_{n}$ and $\sigma_{n}^{\prime}$ when $n=n_{\ell}-k(\ell)$. Here the orbit of the coordinate $x_{i_{1}}$ is in the fake Rokhlin tower $\tilde{C}_{n}$ on the interval $\tilde{J}_{n}+\left(r_{0}-1\right) h_{n}$, whereas on the same interval the orbit of the coordinate $x_{i_{2}}$ is in $C_{n}$.

\subsection{The case $n_{\ell}-k(\ell)<n<n_{\ell}$}

It only remains now to study the case where, for each $\ell$ large enough and each $n_{\ell-1} \leqslant n \leqslant n_{\ell}-k(\ell)$, all substantial $n$-crossings are synchronized, but still there exist infinitely many integers $n$ for which at least one substantial $n$-crossing is not synchronized.

We consider now an integer $n$ with $n_{\ell}-k(\ell)<n<n_{\ell}$ for some large $\ell$, and we assume that there is at least one substantial $n$-crossing which is not synchronized. We can also assume that for each $n_{(\ell-1)} \leqslant m \leqslant n-1$, all substantial $m$-crossings are synchronized. Then, as in Section 7.2 , we construct a family $\left(J_{m}\right)$ of intervals, $n_{\ell-1} \leqslant m \leqslant n$, where $J_{m}$ is the $m$-crossing containing 0 , and is of size $\left|J_{m}\right| \geqslant$ $h_{m}-(d+2) \eta h_{n_{(\ell-1)}}$. We set $n^{\prime}:=n_{\ell}-k(\ell)$. We have $\left|J_{n^{\prime}}\right| \geqslant(1-\eta / 100) h_{n^{\prime}}$, provided $\ell$ is large enough.

As in Section 7.3, we apply Lemma 7.3 for $n^{\prime}$. We consider all integers $r \geqslant 0$ such that $r h_{n^{\prime}} \leqslant 4 h_{n}$ : each such integer $r$ corresponds either to a true $n^{\prime}$-crossing (if for each $i=1, \ldots, d, T^{r h_{n^{\prime}}} x_{i} \in C_{n^{\prime}}$ ), or to a fake $n^{\prime}$-crossing (if there exists $i$ such that $\left.T^{r h_{n^{\prime}}} x_{i} \in \tilde{C}_{n^{\prime}}\right)$. If $T^{r h_{n^{\prime}}} x_{i} \in C_{n^{\prime}}$, then we can consider $t_{n^{\prime}}\left(T^{r h_{n^{\prime}}} x_{i}\right)$ which evolves according to the rules stated in Section 7.3. We can even precise a little bit more these rules by considering also the position of $T^{j} x_{i}$ relatively to tower $n$ : If $T^{r h_{n^{\prime}}} x_{i}$ is in the fake tower $n^{\prime}$, then $T^{(r-1) h_{n^{\prime}}} x_{i}$ and $T^{(r+1) h_{n^{\prime}}} x_{i}$ are in $C_{n^{\prime}} \subset C_{n}$, and we have $t_{n^{\prime}}\left(T^{(r-1) h_{n^{\prime}}} x_{i}\right)=t_{n}\left(T^{(r-1) h_{n^{\prime}}} x_{i}\right)=3$, and $t_{n^{\prime}}\left(T^{(r+1) h_{n^{\prime}}} x_{i}\right)=t_{n}\left(T^{(r+1) h_{n^{\prime}}} x_{i}\right)=1$.

Let us first consider the case where each $0 \leqslant r \leqslant\left\lfloor 4 h_{n} / h_{n^{\prime}}\right\rfloor$ corresponds to a true $n^{\prime}$-crossing. Then the interval $\left\{0, \ldots, 4 h_{n}\right\}$ is contained in a single $n_{\ell^{-}}$crossing. We denote by $\tilde{J}_{n}$ the interval obtained by removing from $J_{n}$ its first 3 points. Then, as in the proof of Lemma 7.3 , we prove that $0 \in \tilde{J}_{n}$, and that the intervals $\tilde{J}_{n}$, $\tilde{J}_{n}+h_{n}, \tilde{J}_{n}+2 h_{n}, \tilde{J}_{n}+3 h_{n}$ are each contained in some $n$-crossing. Since $J_{n}$ is not 
synchronized, we show by similar arguments as in the proof of Lemma 7.6 that for some $s \in\{0,1,2\}$,

$$
\{1,2\} \subset\left\{t_{n}\left(T^{s h_{n} x_{i}}\right): i=1, \ldots, d\right\} .
$$

Then we construct the measures $\sigma_{n}:=\gamma_{\tilde{J}_{n}+s h_{n}}$ and $\sigma_{n}^{\prime}:=\gamma_{\tilde{J}_{n}+(s+1) h_{n}}$ : by similar arguments as before, we construct a twisting transformation $S_{n}$ such that (7.1) holds whenever $B$ is an $m$-box in $\Omega_{m}$ for some $m \leqslant n$. If this can be done for infinitely many integers $n$, then Proposition 5.7 shows that $\sigma_{n} \underset{n \rightarrow \infty}{\longrightarrow} \sigma$ and $\sigma_{n}^{\prime} \underset{n \rightarrow \infty}{\longrightarrow} \sigma$. Then Proposition 6.3 shows that $\sigma$ can be decomposed as a product of two Radon measures to which we can apply the induction hypothesis.

Now we consider the case where there exists some $r, 1 \leqslant r \leqslant\left\lfloor 4 h_{n} / h_{n^{\prime}}\right\rfloor$, which corresponds to a fake $n^{\prime}$-crossing. This case is illustrated on Figure 7.3. We define $r_{0}$ as the smallest integer with this property. Then we know that there exists $i \in\{1, \ldots, d\}$ such that $T^{r_{0} h_{n^{\prime}}} x_{i} \in \tilde{C}_{n^{\prime}}$. Each such $i$ is called an outgoing coordinate. Note that for each outgoing coordinate $i$, we have for each $n^{\prime} \leqslant m \leqslant n t_{m}\left(T^{\left(r_{0}-1\right) h_{n^{\prime}}} x_{i}\right)=3$ (indeed, the orbit of the outgoing coordinate has to reach the top of tower $m$ before leaving $\left.C_{n_{\ell}}\right)$.

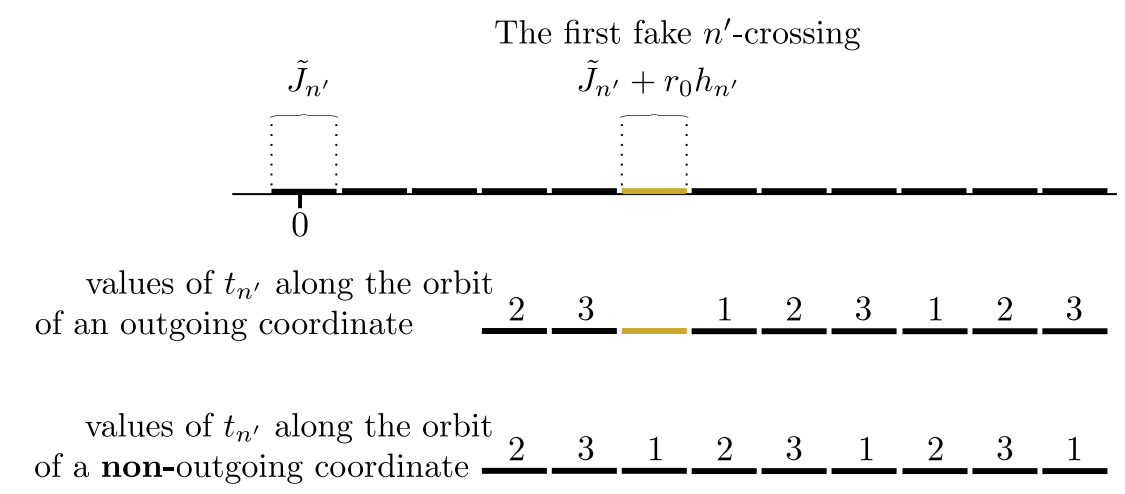

Figure 7.3. The behaviour of $t_{n^{\prime}}$ along the orbits of outgoing and non-outgoing coordinates

We also observe that, since the interval $\left\{0, \ldots,\left(r_{0}-1\right) h_{n^{\prime}}\right\}$ is contained in an

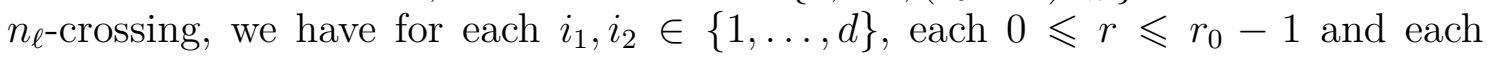
$n^{\prime} \leqslant m \leqslant n$

$$
t_{m}\left(T^{r h_{n^{\prime}}} x_{i_{1}}\right)-t_{m}\left(T^{r h_{n^{\prime}}} x_{i_{2}}\right)=t_{m}\left(x_{i_{1}}\right)-t_{m}\left(x_{i_{2}}\right) .
$$

For $n^{\prime} \leqslant m<n$, the above difference vanishes. Hence, we have $t_{m}\left(T^{\left(r_{0}-1\right) h_{n^{\prime}}} x_{i_{1}}\right)=$ $t_{m}\left(T^{\left(r_{0}-1\right) h_{n^{\prime}}} x_{i_{2}}\right)$ for each $i_{1}, i_{2}$. Taking into account the outgoing coordinates, we see that for each $i=1, \ldots, d, t_{m}\left(T^{\left(r_{0}-1\right) h_{n^{\prime}}} x_{i}\right)=3$. This proves that at time $\left(r_{0}-1\right) h_{n^{\prime}}$, each coordinate is in the last occurrence of tower $n^{\prime}$ inside tower $n$.

Now, since the $n$-crossing $J_{n}$ containing 0 is not synchronized, there exist $i_{1}, i_{2}$ such that the difference in (7.4) does not vanish for $m=n$, and this implies that there exist some $i \in\{1, \ldots, d\}$ such that $t_{n}\left(T^{\left(r_{0}-1\right) h_{n^{\prime}}} x_{i}\right) \neq 3$. In particular such an $i$ is not an outgoing coordinate. At time $r_{0} h_{n^{\prime}}$, the orbit of a non-outgoing coordinate is in the first occurrence of tower $n^{\prime}$ inside tower $n$. When $r$ runs over the set $R:=\left\{r_{0}, \ldots, r_{0}+3^{n-n^{\prime}}-1\right\}$, we get that for each non-outgoing coordinate $i, T^{r h_{n^{\prime}}} x_{i}$ 
successively belongs to successive occurrences of tower $n^{\prime}$ inside tower $n$, and we have $t_{n^{\prime}}\left(T^{r h_{n^{\prime}}} x_{i}\right)=r-r_{0}+1 \bmod 3$.

On the other hand, if $i$ is an outgoing coordinate, the orbit of $x_{i}$ falls into the first occurrence of tower $n^{\prime}$ inside tower $n$ only at time $\left(r_{0}+1\right) h_{n}$. And we have, for $r \in R \backslash\left\{r_{0}\right\}, t_{n^{\prime}}\left(T^{r h_{n^{\prime}}} x_{i}\right)=r-r_{0} \bmod 3$.

Set $R_{1}:=\left\{r \in R: r-r_{0}=1 \bmod 3\right\}$. Then $R_{1} \neq \emptyset$ because $n>n^{\prime}$, and for $r \in R_{1}$, we have

$$
t_{n^{\prime}}\left(T^{r h_{n^{\prime}}} x_{i}\right)= \begin{cases}1 & \text { if } i \text { is an outgoing coordinate } \\ 2 & \text { otherwise. }\end{cases}
$$

Let $\tilde{J}_{n^{\prime}}$ be the interval obtained after removing the first $4^{\ell}$ points from $J_{n^{\prime}}$, and set $J:=\bigsqcup_{r \in R_{1}} \tilde{J}_{n^{\prime}}+r h_{n^{\prime}}, J^{\prime}:=J+h_{n^{\prime}}$, and let $I$ be the smallest interval containing $J$ and $J^{\prime}$. Then $J$ and $J^{\prime}$ have inside $I$ the structure required in Proposition 5.9, with $M=3$. We consider the two measures $\sigma_{n}:=\gamma_{J}$ and $\sigma_{n}^{\prime}:=\gamma_{J^{\prime}}$. Then there exists a twisting transformation $S_{n}$, defined from the partition of $\{1, \ldots, d\}$ into outgoing and non-outgoing coordinates, such that (7.1) holds whenever $B$ is an $m$-box in $\Omega_{m}$ for some $m \leqslant n^{\prime}$.

If we have infinitely many integers $n$ for which the above construction is possible, then Proposition 5.7 ensures that $\gamma_{I} \underset{n \rightarrow \infty}{\longrightarrow} \sigma$, then Proposition 5.9 yields $\sigma_{n} \underset{n \rightarrow \infty}{\longrightarrow} \sigma$ and $\sigma_{n}^{\prime} \underset{n \rightarrow \infty}{\longrightarrow} \sigma$. Finally, Proposition 6.3 shows that $\sigma$ can be decomposed as a product of two Radon measures to which we can apply the induction hypothesis.

This concludes the proof of Theorem 3.10.

\section{Further properties and a question}

We now provide some direct consequences of our main result on the commutant and factors of the nearly finite Chacon transformation $T$. The proof of the following proposition is directly derived from Section 5 of [JRdlR18] (see in particular Remark 5.7 therein).

Proposition 8.1. -

- If $S$ is an invertible $\mu$-preserving transformation of $X$ commuting with $T$, then $S=T^{k}$ for some $k \in \mathbb{Z}$.

- If $\pi:(X, \mu, T) \rightarrow(Y, \nu, R)$ is a factor map to another invertible, $\sigma$-finite measure preserving dynamical system $(Y, \nu, R)$, then $\pi$ is in fact an isomorphism between the two systems.

For some applications in the study of Poisson suspensions developed in [JRdlR17], we also need an extra property which is the existence of a measurable law of large numbers.

Definition 8.2. - A measurable law of large numbers for a conservative, ergodic, measure preserving dynamical system $(X, \mathscr{A}, \mu, T)$ is a measurable function $L$ : $\{0,1\}^{\mathbb{N}} \rightarrow[0, \infty]$ such that for all $B \in \mathscr{A}$, for $\mu$-almost every $x \in X$,

$$
L\left(\mathbb{1}_{B}(x), \mathbb{1}_{B}(T x), \ldots\right)=\mu(B) .
$$


Definition 8.3. - A conservative, ergodic, measure preserving dynamical system $(X, \mathscr{A}, \mu, T)$ is rationally ergodic if there exists a set $B \in \mathscr{A}, 0<\mu(B)<\infty$, and a constant $M>0$ such that, for any $r \geqslant 1$,

$$
\int_{B}\left(\sum_{0 \leqslant j \leqslant r-1} \mathbb{1}_{B}\left(T^{j} x\right)\right)^{2} \mathrm{~d} \mu(x) \leqslant M\left(\int_{B} \sum_{0 \leqslant j \leqslant r-1} \mathbb{1}_{B}\left(T^{j} x\right) \mathrm{d} \mu(x)\right)^{2} .
$$

According to Theorem 3.3.1 in [Aar97], a measurable law of large numbers exists for $T$ as soon as $T$ is rationally ergodic.

Proposition 8.4. - The nearly finite Chacon transformation is rationally ergodic, hence admits a measurable law of large numbers.

Proof. - In the cutting-and-stacking construction of the Nearly Finite Chacon transformation, we always cut the tower into 3 subcolumns, hence $T$ is rank one with bounded cuts. But it is proved in $\left[\mathrm{BSS}^{+} 15\right.$, Theorem 2.3] that this property implies that $T$ is rationally ergodic.

Let us finally mention the following observation. The original definition by Rudolph [Rud79] of minimal self joinings for finite measure preserving transformations considered any ergodic $T^{\ell_{1}} \times \cdots \times T^{\ell_{d}}$-invariant measure on the $d$-fold Cartesian product for $\ell_{1}, \ldots, \ell_{d} \in \mathbb{Z} \backslash\{0\}$. Although the terminology which was finally adopted refers only to $T^{\otimes d}$-invariant measures, it is nevertheless interesting to consider the action of $T^{\ell_{1}} \times \cdots \times T^{\ell_{d}}$. In the context of the nearly finite Chacon transformation, we can then ask whether there exists, for some $d$ and some $\ell_{1}, \ldots, \ell_{d} \in \mathbb{Z} \backslash\{0\}$, an ergodic $T^{\ell_{1}} \times \cdots \times T^{\ell_{d}}$-invariant measure (say, supported on $\left.X_{\infty}^{d}\right)$ different than those described in the statement of Theorem 3.10.

\section{Acknowledgements}

The authors would like to thank Sasha Danilenko for several discussions on the subject, as well as the anonymous referee for valuable suggestions.

\section{BIBLIOGRAPHY}

[Aar97] Jon Aaronson, An introduction to infinite ergodic theory, Mathematical Surveys and Monographs, vol. 50, American Mathematical Society, 1997. $\uparrow 413$

[AFS97] Terrence Adams, Nathaniel Friedman, and Cesar E. Silva, Rank-one weak mixing for nonsingular transformations, Isr. J. Math. 102 (1997), 269-281. $\uparrow 370$

$\left[\mathrm{BSS}^{+} 15\right]$ Francisc Bozgan, Anthony Sanchez, Cesar E. Silva, David Stevens, and Jane Wang, Subsequence bounded rational ergodicity of rank-one transformations, Dyn. Syst. 30 (2015), no. 1, 70-84. $\uparrow 413$

[Dan18] Alexandre I. Danilenko, Infinite measure preserving transformations with Radon MSJ., Isr. J. Math. 228 (2018), no. 1, 21-51. $\uparrow 369,370$

[dJRS80] Andres del Junco, Maurice Rahe, and Laif Swanson, Chacon's automorphism has minimal self-joinings, J. Anal. Math. 37 (1980), 276-284. $\uparrow 370$

[FS67] Ciprian Foias and Serban Stratila, Ensembles de Kronecker dans la Théorie ergodique, C. R. Math. Acad. Sci. Paris 267 (1967), 166-168. $\uparrow 370$ 
[Hal50] Paul R. Halmos, Measure Theory, D. Van Nostrand Company, 1950. $\uparrow 377$

[JRdIR17] Élise Janvresse, Emmanuel Roy, and Thierry de la Rue, Poisson suspensions and SuShis, Ann. Sci. Éc. Norm. Supér. 50 (2017), no. 6, 1301-1334. ^370, 371, 412

[JRdIR18] _ Invariant measures for Cartesian powers of Chacon infinite transformation, Isr. J. Math. 224 (2018), 1-37. $3699,370,372,381,399,412$

[LPT00] Mariusz Lemańczyk, François Parreau, and Jean-Paul Thouvenot, Gaussian automorphisms whose ergodic self-joinings are Gaussian, Fundam. Math. 164 (2000), no. 3, 253-293. $\uparrow 370$

[Roy07] Emmanuel Roy, Ergodic properties of Poissonian ID processes, Ann. Probab. 35 (2007), no. $2,551-576 . \uparrow 370$

[Rud79] Daniel J. Rudolph, An example of a measure preserving map with minimal self-joinings, and applications, J. Anal. Math. 35 (1979), 97-122. $\uparrow 413$

Manuscript received on 16th February 2018, revised on 24th January 2019, accepted on 22nd February 2019.

Recommended by Editor Y. Coudène. Published under license CC BY 4.0.

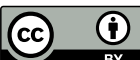

This journal is a member of Centre Mersenne.

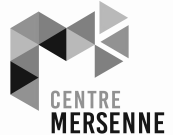

Élise JANVRESSE

Laboratoire Amiénois de Mathématique Fondamentale et Appliquée, CNRS-UMR 7352

Université de Picardie Jules Verne

33 rue Saint Leu

80039 Amiens cedex 1 (France)

elise.janvresse@u-picardie.fr

Emmanuel ROY

Laboratoire Analyse, Géométrie et Applications

Université Paris 13 Institut Galilée

99 avenue Jean-Baptiste Clément

93430 Villetaneuse (France)

roy@math.univ-paris13.fr

Thierry DE LA RUE

Laboratoire de Mathématiques Raphaël Salem

Université de Rouen Normandie, CNRS

Avenue de l'Université

76801 Saint Étienne du Rouvray (France)

thierry.de-la-rue@univ-rouen.fr 